DOI 10.4171/JEMS/364

Andrea Bonfiglioli · Ermanno Lanconelli

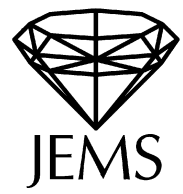

\title{
Subharmonic functions in sub-Riemannian settings
}

Received December 17, 2010 and in revised form February 7, 2011

\begin{abstract}
In this paper we furnish mean value characterizations for subharmonic functions related to linear second order partial differential operators with nonnegative characteristic form, possessing a well-behaved fundamental solution $\Gamma$. These characterizations are based on suitable average operators on the level sets of $\Gamma$. Asymptotic characterizations are also considered, extending classical results of Blaschke, Privaloff, Radó, Beckenbach, Reade and Saks. We analyze as well the notion of subharmonic function in the sense of distributions, and we show how to approximate subharmonic functions by smooth ones. The classes of operators involved are wide enough to contain, as very special cases, the sub-Laplacians on Carnot groups. The results presented here generalize and carry forward former results of the authors in $[6,9]$.
\end{abstract}

\section{Contents}

1. Introduction. $\mathcal{L}$-subharmonic functions . . . . . . . . . . . . . . . . . . . . . 387

2. Assumptions on the operator $\mathcal{L}$. The $\mathcal{L}$-harmonic space $\ldots \ldots \ldots \ldots$. . . . . . . 392

3. Level sets of $\Gamma$. Average operators, representation formulas . . . . . . . . . . . . . . . . 394

4. Mean value characterizations of $\mathcal{L}$-subharmonic functions: Main Theorem . . . . . . . . 398

5. A Kozakiewicz-type theorem. Proof of Main Theorem . . . . . . . . . . . . . . . . . . . 399

6. $\mathcal{L}$-subharmonicity of the average operators . . . . . . . . . . . . . . . . . . . 409

7. Smoothing of $\mathcal{L}$-subharmonic functions . . . . . . . . . . . . . . . . . . 416

8. $\mathcal{L}$-subharmonic functions in the sense of distributions . . . . . . . . . . . . . 417

9. The case of sub-Laplacians: Improved results . . . . . . . . . . . . . . . . . . . 420

10. Saks-type theorems . . . . . . . . . . . . . . . . . . . 426

11. Appendix. Proof of the $\mathcal{L}$-representation formulas . . . . . . . . . . . . . . . 432

References . . . . . . . . . . . . . . . . . . . . . . 440

\section{Introduction. $\mathcal{L}$-subharmonic functions}

Let

$$
\mathcal{L}:=\sum_{i, j=1}^{N} \partial_{x_{i}}\left(a_{i, j}(x) \partial_{x_{j}}\right)=\operatorname{div}(A(x) \nabla)
$$

A. Bonfiglioli, E. Lanconelli: Dipartimento di Matematica, Università di Bologna, Piazza di Porta S. Donato, 5, 40126 Bologna, Italy;

e-mail: andrea.bonfiglioli6@unibo.it, ermanno.lanconelli@unibo.it

Mathematics Subject Classification (2010): Primary 31B05, 35H20; Secondary 35J70 
be a linear second order PDO in $\mathbb{R}^{N}$, in divergence form, with $C^{2}$ coefficients and such that the matrix $A(x):=\left(a_{i, j}(x)\right)_{i, j \leq N}$ is symmetric and nonnegative definite at any point $x=\left(x_{1}, \ldots, x_{N}\right) \in \mathbb{R}^{N}$. In $(1.1), \nabla$ denotes the usual Euclidean gradient operator $\nabla=$ $\left(\partial_{x_{1}}, \ldots, \partial_{x_{N}}\right)^{T}$.

The operator $\mathcal{L}$ is (possibly) degenerate elliptic. However, in addition to some general hypotheses that will be fixed in the sequel, throughout the paper we always assume without further comments that $\mathcal{L}$ is not totally degenerate at every point. Precisely, we assume that the following condition holds:

(ND) There exists $i \in\{1, \ldots, N\}$ such that $a_{i, i}>0$ on $\mathbb{R}^{N}$.

This condition, together with $A(x) \geq 0$, implies Picone's Maximum Principle for $\mathcal{L}$ :

If $V \subset \mathbb{R}^{N}$ is open and bounded and $u \in C^{2}(V, \mathbb{R})$ satisfies

$$
\mathcal{L} u \geq 0 \quad \text { in } V \quad \text { and } \quad \limsup _{x \rightarrow y} u(x) \leq 0 \quad \text { for every } y \in \partial V,
$$

then $u \leq 0$ in $V$. (See [22, Corollary 1.3].)

A function $h$ will be said $\mathcal{L}$-harmonic in an open set $\Omega \subseteq \mathbb{R}^{N}$ if $h \in C^{2}(\Omega, \mathbb{R})$ and $\mathcal{L} h=0$ in $\Omega$. An upper semicontinuous function (u.s.c. function, for short) $u: \Omega \rightarrow$ $[-\infty, \infty)$ will be called $\mathcal{L}$-subharmonic in $\Omega$ if

(i) the set $\Omega(u):=\{x \in \Omega: u(x)>-\infty\}$ contains at least one point of every (connected) component of $\Omega$, and

(ii) for every bounded open set $V \subset \bar{V} \subset \Omega$ and for every $\mathcal{L}$-harmonic function $h \in$ $C^{2}(V, \mathbb{R}) \cap C(\bar{V}, \mathbb{R})$ such that $u \leq h$ on $\partial V$, one has $u \leq h$ in $V$.

We shall denote by $\underline{\mathcal{S}}_{\mathcal{L}}(\Omega)$, or simply by $\mathcal{S}(\Omega)$, the family (actually, the cone) of $\mathcal{L}$-subharmonic functions in $\Omega$.

It is well known that the subharmonic functions play crucial rôles in potential theory of linear second order PDE's (just think about Perron's method for the Dirichlet problem) as well as in studying the notion of convexity in Euclidean and non-Euclidean settings. (See the bibliographical notes at the end of the introduction for some related references.)

When $\mathcal{L}$ is the classical Laplace operator $\Delta$, several characterizations of the $\Delta$-subharmonicity, involving surface and solid average operators on Euclidean balls, are given in the literature. Some of them are quite well known, others are less so. If $H_{\alpha}$ is the $\alpha$-dimensional Hausdorff measure, and if we denote by

$$
\begin{aligned}
& \mathbf{S}_{r}(u)(x):=\frac{1}{H_{N-1}(\partial B(x, r))} \int_{\partial B(x, r)} u(y) \mathrm{d} H_{N-1}(y) \quad \text { and } \\
& \mathbf{B}_{r}(u)(x):=\frac{1}{H_{N}(B(x, r))} \int_{B(x, r)} u(y) \mathrm{d} H_{N}(y)
\end{aligned}
$$

respectively, the mean value operator on the Euclidean sphere of center $x$ and radius $r$, and on the corresponding solid ball $B(x, r)$, we can list the previously mentioned characterizations as follows. 
Theorem A. Let $\Omega \subseteq \mathbb{R}^{N}$ be an open set and let $u: \Omega \rightarrow[-\infty, \infty)$ be an u.s.c. function such that $\Omega(u)$ contains at least one point of every component of $\Omega$. Given $x \in \Omega$, set $R(x):=\sup \{r>0: \overline{B(x, r)} \subset \Omega\}$. Then the following statements are equivalent:

(i) $u \in \underline{\mathcal{S}}_{\Delta}(\Omega)$.

(ii) For every $x \in \Omega$ and $0<r<R(x)$, we have $u(x) \leq \mathbf{S}_{r}(u)(x)$.

(iii) For every $x \in \Omega$ and $0<r<R(x)$, we have $u(x) \leq \mathbf{B}_{r}(u)(x)$.

(iv) (Blaschke) For every $x \in \Omega(u)$, we have

$$
\limsup _{r \rightarrow 0} \frac{\mathbf{S}_{r}(u)(x)-u(x)}{r^{2}} \geq 0 .
$$

(v) (Privaloff) For every $x \in \Omega(u)$, we have

$$
\limsup _{r \rightarrow 0} \frac{\mathbf{B}_{r}(u)(x)-u(x)}{r^{2}} \geq 0 .
$$

(vi) For every $x \in \Omega$, the function $r \mapsto \mathbf{S}_{r}(u)(x)$ is increasing on $(0, R(x))$ and $\lim _{r \rightarrow 0} \mathbf{S}_{r}(u)(x)=u(x)$.

(vii) For every $x \in \Omega$, the function $r \mapsto \mathbf{B}_{r}(u)(x)$ is increasing on $(0, R(x))$ and $\lim _{r \rightarrow 0} \mathbf{B}_{r}(u)(x)=u(x)$.

(viii) (Beckenbach-Radó) For every $x \in \Omega$ and $r \in(0, R(x))$, we have $\mathbf{B}_{r}(u)(x) \leq$ $\mathbf{S}_{r}(u)(x)$ and $\lim _{r \rightarrow 0} \mathbf{S}_{r}(u)(x)=u(x)$.

(ix) (Reade) For every $x \in \Omega(u)$, we have

$$
\liminf _{r \rightarrow 0} \frac{\mathbf{S}_{r}(u)(x)-\mathbf{B}_{r}(u)(x)}{r^{2}} \geq 0 \quad \text { and } \quad \lim _{r \rightarrow 0} \mathbf{S}_{r}(u)(x)=u(x) .
$$

Sharp versions of Blaschke and Privaloff conditions were proved by Saks.

If $u$ is $\Delta$-subharmonic in $\Omega$ then, by Riesz's Representation Theorem, there exists a Radon measure $\mu_{u}$ (called the Riesz measure of $u$ ) such that $\Delta u=\mu_{u}$ in $\mathcal{D}^{\prime}(\Omega)$. On the other hand, from the Lebesgue Differentiation Theorem for measures, the symmetric derivative of $\mu_{u}$,

$$
D_{\mathrm{s}} \mu_{u}(x):=\lim _{r \rightarrow 0} \frac{\mu_{u}(B(x, r))}{H_{N}(B(x, r))},
$$

exists $H_{N}$-almost everywhere in $\Omega$. The following result holds.

Theorem B (Saks). Let $u$ be a $\Delta$-subharmonic function in $\Omega \subseteq \mathbb{R}^{N}$ and let $\mu_{u}$ be its Riesz measure. Then, at every point $x \in \Omega$ where $D_{\mathrm{s}} \mu_{u}(x)$ exists, one has:

(i) $\lim _{r \rightarrow 0} \frac{\mathbf{S}_{r}(u)(x)-u(x)}{r^{2}}=\frac{1}{2 N} D_{\mathrm{s}} \mu_{u}(x)$,

(ii) $\lim _{r \rightarrow 0} \frac{\mathbf{B}_{r}(u)(x)-u(x)}{r^{2}}=\frac{1}{2(N+2)} D_{\mathrm{s}} \mu_{u}(x)$,

(iii) $\lim _{r \rightarrow 0} \frac{\mathbf{S}_{r}(u)(x)-\mathbf{B}_{r}(u)(x)}{r^{2}}=\frac{1}{N(N+2)} D_{\mathrm{s}} \mu_{u}(x)$.

Finally, we want to recall the following theorem, resting on the hypoellipticity of $\Delta$. 
Theorem C. Let $\Omega \subseteq \mathbb{R}^{N}$ be open and let $u \in L_{\mathrm{loc}}^{1}(\Omega)$. Then:

(i) $u \in \underline{\mathcal{S}}_{\Delta}(\Omega)$ if and only if $\Delta u \geq 0$ in the sense of distributions on $\Omega$ and $\lim _{r \rightarrow 0} \mathbf{B}_{r}(u)(x)=u(x)$ for every $x \in \Omega$;

(ii) if $u \in \underline{\mathcal{S}}_{\Delta}(\Omega)$ and $\Omega^{\prime}$ is an open subset of $\Omega$ with positive distance from $\partial \Omega$, there exists an increasing sequence $\left\{u_{n}\right\}_{n \in \mathbb{N}}$ of smooth $\Delta$-subharmonic functions in $\Omega^{\prime}$ such that $\lim _{n \rightarrow \infty} u_{n}(x)=u(x)$ for every $x \in \Omega^{\prime}$.

Remark 1.1 (Viscosity characterization of $\Delta$-subharmonicity). For completeness, we would like to add to the previous theorems the following viscosity characterization of the classical $\Delta$-subharmonic functions:

An u.s.c. function $u$ is $\Delta$-subharmonic in the open set $\Omega$ if and only if for every $C^{2}$ function $\phi$ such that $u-\phi$ has a local maximum at a point $x_{0} \in \Omega$ we have $\Delta \phi\left(x_{0}\right) \geq 0$.

The goal of this paper is to recast Theorems A, B and C above in more general settings, today usually called of sub-Riemannian type. (We notice that the proposition in Remark 1.1 , that is, the viscosity characterization of subharmonic functions, has already been extended in [23] to a class of linear second order PDO's with nonnegative characteristic form. The class in [23] is wider than the ones considered in the present paper.)

To be more specific, we extend Theorem A to every operator $\mathcal{L}$ endowing $\mathbb{R}^{N}$ with the structure of a $\mathfrak{S}^{*}$-harmonic space, and having a nonnegative global fundamental solution

$$
\mathbb{R}^{N} \times \mathbb{R}^{N} \backslash\{x=y\} \ni(x, y) \mapsto \Gamma(x, y) \in \mathbb{R},
$$

with pole at any point of the diagonal $\{x=y\}$ of $\mathbb{R}^{N}$. (For the notion of $\mathfrak{S}^{*}$-harmonic space, see [9, Section 6.10].)

In our version of Theorem A the classical mean value operators $\mathbf{S}_{r}$ and $\mathbf{B}_{r}$ are replaced by suitable average operators on the level sets of $\Gamma$,

$$
\partial \Omega_{r}(x)=\left\{y \in \mathbb{R}^{N}: \Gamma(x, y)=1 / r\right\},
$$

and on their solid counterpart $\Omega_{r}(x):=\left\{y \in \mathbb{R}^{N}: \Gamma(x, y)>1 / r\right\}$.

We explicitly remark that study of the integral operators related to the general PDO's considered in this paper is complicated by the presence of nontrivial kernels. For instance, when $\mathcal{L}$ in (1.1) is a sub-Laplacian on a stratified Lie group $\mathbb{G}$, the kernels appearing in the relevant mean-integrals cannot be identically 1, unless $\mathbb{G}$ is the usual Euclidean group $\left(\mathbb{R}^{N},+\right)$, as proved in [7].

A crucial tool for our extension of Theorem A is Theorem 5.3 in Section 5, which provides a unifying approach to several characterizations of $\mathcal{L}$-subharmonicity. Theorem 5.3 traces back to a 1933 theorem by W. Kozakiewicz [21] related to the case of the ordinary Laplace operator.

Our versions of Theorems B, C require an extra assumption: the hypoellipticity of $\mathcal{L}$.

Moreover, in order to generalize Theorem B, we impose a further restriction. Indeed, our approach to this last extension exploits Poisson-Jensen formulas for $\mathcal{L}$-subharmonic functions, together with a homogeneity property for the measure of the level sets of $\Gamma$. Therefore, Theorem B can be conveniently extended to the sub-Laplacians on stratified 
Lie groups, which naturally satisfy these requirements. [Assertion (iii) in Theorem B is an obvious consequence of (i) and (ii). However we extend (iii) to the sub-Laplacians by using a new Poisson-Jensen type formula, that we prove in Theorem 10.2. This new formula seems to be of independent interest.]

We stress that our Saks-type theorem implies a kind of almost-everywhere secondorder differentiability (as in formula (10.16)) for the v-convex functions on Carnot groups $\mathbb{G}$. Indeed, the v-convex functions are $\mathcal{L}$-subharmonic with respect to every subLaplacian $\mathcal{L}$ on $\mathbb{G}$ (for this last fact and for the notion of v-convexity, see [20, 24]). Other remarks on this subject are contained in [8].

While we directly refer to the table of contents for a detailed plan of the arguments presented in this paper, here we only mention that, for the reader's convenience, we have moved to the Appendix the proofs of the $\mathcal{L}$-representation formulas for $C^{2}$-functions presented in Section 3, since they are quite laborious.

We close the Introduction with the following bibliographical notes.

Bibliographical notes. Gauss' Theorem on mean value properties for classical harmonic functions has been generalized in countless directions. The historical development of the problems related to this property, both for harmonic and caloric functions, is presented in the survey paper [27] by Netuka and Veselý.

A mean value theorem for solutions to $\mathcal{L} u=0$, when $\mathcal{L}$ is a general operator as in (1.1) of "elliptic-type", has been proved by Hoh and Jacob [18]. Citti, Garofalo and Lanconelli [11] proved some representation formulas for smooth solutions to $\mathcal{L} u=f$, for operators $\mathcal{L}$ which are sums of squares of vector fields satisfying the Hörmander rank condition.

Later on, these formulas were used in [6] to derive representation formulas for smooth solutions to $\Delta_{\mathbb{G}} u=f$, where $\Delta_{\mathbb{G}}$ denotes a sub-Laplacian on a Carnot group $\mathbb{G}$. When $f=0$ and $\Delta_{\mathbb{G}}$ is the Kohn Laplacian on the Heisenberg group, the formulas in [6], as well as those in [11] and [18], give back a mean value property first proved by Gaveau [16].

The use of asymptotic average operators in the characterization of classical subharmonic functions has a long history, starting with the papers [3] and [28] by Blaschke and Privaloff, respectively. Beckenbach and Radó [2] characterized the $\Delta$-subharmonic functions in terms of the inequality "solid average $\leq$ surface average". [The original Beckenbach-Radó condition was stated and proved for continuous functions in $\mathbb{R}^{2}$. An extension to any dimension, still for continuous functions, is contained in the very recent paper [14] by Freitas and Matos.]

It was Saks [31] who proved, in 1941, Theorem B. Two years later, Reade [29] introduced his asymptotic version of the Beckenbach-Radó condition. [We call the condition contained in item (ix) of Theorem A the Reade condition. Actually, Reade stated in [29], but without any proof, that a continuous function $u$ is $\Delta$-subharmonic in an open set $\Omega \subseteq \mathbb{R}^{2}$ iff $\lim \sup _{r \rightarrow 0}\left(\mathbf{S}_{r}(u)(x)-\mathbf{B}_{r}(u)(x)\right) / r^{2} \geq 0$ for every $x \in \Omega$. We have not been able to find any proof of this statement in the literature. Instead, item (ix) of Theorem A follows from our Theorem 4.2 applied to $\Delta$.]

A modern reference for some asymptotic-mean characterizations of $\Delta$-subharmonicity is the monograph [1] by Armitage and Gardiner (see [1, Section 3.2]). This mono- 
graph, which mainly deals with classical potential theory, also contains some applications of subharmonicity to the usual convexity (a systematic subharmonic approach to convexity in the Euclidean setting can also be found in Hörmander's monograph [19]).

Mean value characterizations of subharmonic functions in Carnot groups are contained in [6] (see also the monograph [9, Chapter 8]). We directly refer to this chapter for some applications, and a list of references, about convexity in the stratified Lie group setting. Furthermore, [6] and [9] also deal with the problem of smooth approximation of subharmonic functions in Carnot groups. The results proved therein use a version of Friedrichs's mollifiers, resting on the homogeneous Lie group structure of $\mathbb{G}$. This approach does not work in the absence of such an algebraic structure underlying the operator $\mathcal{L}$. For our approximation theorem in the present paper we exploit an idea used in [15] by Garofalo and one of us, for classical parabolic operators with variable coefficients.

We would like to close these bibliographical notes by quoting a very recent paper by Manfredi, Parviainen and Rossi [25] in which the authors show a remarkable Privalofftype characterization of $p$-harmonic functions, precisely the viscosity solutions to $\Delta_{p} u=$ 0 , where $\Delta_{p}$ is the $p$-Laplacian in $\mathbb{R}^{N}, 1<p \leq \infty$. (We are indebted to Juan Manfredi for bringing to our attention his joint paper [25] with Parviainen and Rossi.)

\section{Assumptions on the operator $\mathcal{L}$. The $\mathcal{L}$-harmonic space}

We assume that the operator $\mathcal{L}$ in (1.1) is equipped with a global fundamental solution $\Gamma$, that is, there exists a function $\Gamma: D=\left\{(x, y) \in \mathbb{R}^{N} \times \mathbb{R}^{N}: x \neq y\right\} \rightarrow \mathbb{R}$ with the following properties:

(G.1) $\Gamma \in L_{\text {loc }}^{1}\left(\mathbb{R}^{N} \times \mathbb{R}^{N}\right) \cap C^{2}(D, \mathbb{R}), \Gamma(x, y)>0$ for every $(x, y) \in D$;

(G.2) for every fixed $x \in \mathbb{R}^{N}$, we have $\lim _{y \rightarrow x} \Gamma(x, y)=\infty$ and $\lim _{y \rightarrow \infty} \Gamma(x, y)=0$;

(G.3) for any $\varphi \in C_{0}^{\infty}\left(\mathbb{R}^{N}, \mathbb{R}\right)$,

$$
\int_{\mathbb{R}^{N}} \Gamma(x, y) \mathcal{L} \varphi(y) \mathrm{d} y=-\varphi(x) \quad \text { for every } x \in \mathbb{R}^{N} .
$$

This last property, since $\mathcal{L}^{*}=\mathcal{L}$, can be restated as follows: $-\mathcal{L} \Gamma(x, \cdot)$ equals the Dirac measure at $\{x\}$, in the sense of distributions. This in particular implies that $y \mapsto \Gamma(x, y)$ is $\mathcal{L}$-harmonic in $\mathbb{R}^{N} \backslash\{x\}$. As a consequence, since $\Gamma(x, y) \rightarrow \infty$ as $y \rightarrow x$, an easy application of Picone's Maximum Principle shows that $-\Gamma(x, \cdot)$ is $\mathcal{L}$-subharmonic in $\mathbb{R}^{N}$.

Our second general assumption is that $\mathcal{L}$ endows $\mathbb{R}^{N}$ with the structure of $\mathfrak{S}^{*}$-harmonic space, in the sense of [9, Definition 6.10.1]. Due to (G.1) and (G.2), this amounts to making the following extra hypotheses on $\mathcal{L}$ :

(D) Doob convergence property: If $\left\{u_{n}\right\}_{n}$ is an increasing sequence of $\mathcal{L}$-harmonic functions on an open set $\Omega \subseteq \mathbb{R}^{N}$, then $u:=\sup _{n} u_{n}$ is $\mathcal{L}$-harmonic in $\Omega$, provided that $u$ is finite in a dense subset of $\Omega$.

(R) Regularity axiom: The $\mathcal{L}$-regular open sets form a basis of the Euclidean topology. 
Here, we agree to define an $\mathcal{L}$-regular set to be any bounded open set $V \subset \mathbb{R}^{N}$ such that: for every $f \in C(\partial V, \mathbb{R})$ there exists a (unique) $\mathcal{L}$-harmonic function in $V$, denoted by $H_{f}^{V}$, satisfying $\lim _{y \rightarrow x} H_{f}^{V}(y)=f(x)$ for every $x \in \partial V$.

Remark 2.1. We want to stress that the $\mathfrak{S}^{*}$ condition guarantees the solvability of the Dirichlet problem for $\mathcal{L}$ on the level sets $\Omega_{r}(x)$ of $\Gamma(x, \cdot)$ (see the beginning of Section $3)$. We shall also need this property in order to define and use the $\mathcal{L}$-harmonic measures on $\partial \Omega_{r}(x)$ (see, e.g., Lemma 5.8). As we shall observe in Remark 2.2 below, conditions (D) and $(\mathrm{R})$ are satisfied if $\mathcal{L}$ is hypoelliptic; however, we shall need this stronger assumption only after Section 6.

Under the previous assumptions, the map

$$
\Omega \mapsto \mathcal{H}(\Omega)=\left\{u \in C^{2}(\Omega, \mathbb{R}): u \text { is } \mathcal{L} \text {-harmonic in } \Omega\right\}
$$

is a harmonic sheaf and $\left(\mathbb{R}^{N}, \mathcal{H}\right)$ is a $\mathfrak{S}^{*}$-harmonic space, which we call the $\mathcal{L}$-harmonic space. Indeed, the functions of the type $\max \{-\Gamma(x, \cdot),-k\}$ (with $k \in \mathbb{N}$ ) provide nonpositive continuous $\mathcal{L}$-subharmonic functions separating points of $\mathbb{R}^{N}$.

Remark 2.2. Conditions (D) and (R) are satisfied if $\mathcal{L}$ is hypoelliptic (see [9, Chapter 7, Exercise 7]). In particular, this holds true if $\mathcal{L}=\sum_{j=1}^{m} X_{j}^{2}$, where the $X_{j}$ 's are smooth vector fields in $\mathbb{R}^{N}$ satisfying the Hörmander rank condition

$$
\operatorname{dim}\left(\operatorname{Lie}\left\{X_{1}, \ldots, X_{m}\right\}(x)\right)=N, \quad \forall x \in \mathbb{R}^{N} .
$$

The hypoellipticity of $\mathcal{L}$, together with its homogeneity with respect to a group of dilations in $\mathbb{R}^{N}$ (in the sense of Folland and Stein [13]), is a sufficient condition for the existence of a function $\Gamma$ satisfying (G.1)-(G.3). This follows by arguing as in [9, Section 5.3]: Indeed, from the hypoellipticity of $\mathcal{L}$, we infer the existence of a "local" fundamental solution (see Trèves [33, Theorems 52.1, 52.2]); by the homogeneity of $\mathcal{L}$, a "local-toglobal" argument can be performed (see Folland [12, Theorem 2.1]). Then one argues as in [9, Section 5.3] to obtain the required properties of $\Gamma$.

Particular examples of hypoelliptic homogeneous operators are the sub-Laplacians on Carnot groups, considered in Section 9.

Remark 2.3. We would like to stress that our assumptions (G.1)-(G.3) on the fundamental solution of $\mathcal{L}$ imply that every $\mathcal{L}$-subharmonic function is finite in a dense subset of its domain.

Indeed, let $u \in \underline{\mathcal{S}}_{\mathcal{L}}(\Omega)$ and assume, towards a contradiction, that $u \equiv-\infty$ in an open set $O \subseteq \Omega$. Then there exists a superlevel set of $\Gamma, \Omega_{r}(x):=\{y: \Gamma(x, y)>1 / r\}$ $\cup\{x\}$, whose closure is contained in $O$. As we shall prove in a moment, it follows that $\Omega_{s}(x) \subseteq O$ whenever $s>r$ and $\overline{\Omega_{s}(x)} \subset \Omega$. A connectedness argument will then prove that $u \equiv-\infty$ on the connected component of $\Omega$ containing $x$, contrary to the definition of $\mathcal{L}$-subharmonic function. To complete the proof, let $s>r$ be such that $\overline{\Omega_{s}(x)} \subset \Omega$. Letting $V:=\Omega_{s}(x) \backslash \overline{\Omega_{r}(x)}$, and, for any $n \in \mathbb{N}$,

$$
h_{n}:=\frac{\sup }{\Omega_{s}(x)} u-n(\Gamma(x, \cdot)-1 / s),
$$

we have the following facts: 
(i) $M:=\sup _{\overline{\Omega_{s}(x)}} u \in \mathbb{R}$ since $u$ is u.s.c., $\overline{\Omega_{s}(x)}$ is compact and $u$ is not identically $-\infty$ in $\overline{\Omega_{s}(x)}$ (otherwise the proof will be trivial);

(ii) $u \leq M \leq h_{n}$ on $\partial \Omega_{s}(x)$;

(iii) $u=-\infty<h_{n}$ on $\partial \Omega_{r}(x)$;

(iv) $h_{n}$ is $\mathcal{L}$-harmonic in $V$.

Then, since $u$ is $\mathcal{L}$-subharmonic, $u \leq h_{n}$ in $V$. Letting $n \rightarrow \infty$, we get $u \equiv-\infty$ in $V$. Hence $u \equiv-\infty$ in $\Omega_{s}(x)=V \cup \overline{\Omega_{r}(x)}$.

If $V$ is any $\mathcal{L}$-regular open set and $x \in V$, the map $C(\partial V, \mathbb{R}) \ni f \mapsto H_{f}^{V}(x) \in \mathbb{R}$ is linear and it is nonnegative on nonnegative $f$ 's. Hence, there exists a unique Radon measure $\mu_{x}^{V}$ on $\partial V$ such that

$$
H_{f}^{V}(x)=\int_{\partial V} f(y) \mathrm{d} \mu_{x}^{V}(y) \quad \text { for every } f \in C(\partial V, \mathbb{R}) .
$$

One says that $\mu_{x}^{V}$ is the $\mathcal{L}$-harmonic measure related to $V$ and $x$.

In what follows, we write $u \in \operatorname{USC}(\Omega)$ if $u: \Omega \rightarrow[-\infty, \infty)$ is upper semicontinuous in $\Omega$. We explicitly remark that a function $u$ is subharmonic in the sense of the $\mathcal{L}$-harmonic space $\left(\mathbb{R}^{N}, \mathcal{L}\right)$ if and only if it is $\mathcal{L}$-subharmonic according to the definition given in Section 1 above (see [9, Definition 6.5.1 and Theorem 6.5.2]).

Without any further comment, throughout the paper we assume that the operator $\mathcal{L}$ in (1.1) satisfies assumptions (ND), (G.1), (G.2), (G.3), (D) and (R) introduced above.

\section{Level sets of $\Gamma$. Average operators, representation formulas}

We introduce a family of sets which will play a central rôle throughout the paper. For every given $x \in \mathbb{R}^{N}$ and $r>0$, we set

$$
\Omega_{r}(x):=\left\{y \in \mathbb{R}^{N}: \Gamma(x, y)>1 / r\right\} \cup\{x\} .
$$

We shall frequently make the convenient definition $\Gamma(x, x):=\infty$, so that we shall also write $\Omega_{r}(x)=\left\{y \in \mathbb{R}^{N}: \Gamma(x, y)>1 / r\right\}$. For simplicity, we assume that, for every $x \in \mathbb{R}^{N}$ and $r>0, \nabla(\Gamma(x, \cdot)) \neq 0$ on $\partial \Omega_{r}(x)$, whence $\partial \Omega_{r}(x)$ is a smooth manifold of class $C^{2}$. Thanks to Sard's Theorem, this hypothesis could be easily removed (in this case, all the following results will hold for almost every $r$ ). We also have

$$
\partial \Omega_{r}(x)=\left\{y \in \mathbb{R}^{N}: \Gamma(x, y)=1 / r\right\} .
$$

Note that any $\Omega_{r}(x)$ is a bounded open neighborhood of $x$ and

$$
\bigcap_{r>0} \Omega_{r}(x)=\{x\}, \quad \bigcup_{r>0} \Omega_{r}(x)=\mathbb{R}^{N}
$$

Here and below, if $E$ is any (Lebesgue) measurable subset of $\mathbb{R}^{N}$, we denote by $|E|$ its Lebesgue measure. Moreover, $\mathrm{d} y$ and $\mathrm{d} \sigma(y)$ will respectively denote the Lebesgue measure and the surface measure in $\mathbb{R}^{N}$, the latter being the Hausdorff $(N-1)$-dimensional measure. 
We claim that, with the above notation, for any $x \in \mathbb{R}^{N}$,

$$
\lim _{r \rightarrow 0} \frac{\left|\Omega_{r}(x)\right|}{r}=0
$$

Indeed, since $\Gamma \in L_{\text {loc }}^{1}$, we have

$$
\frac{\left|\Omega_{r}(x)\right|}{r}=\frac{1}{r} \int_{\Omega_{r}(x)} \mathrm{d} y \leq \int_{\Omega_{r}(x)} \Gamma(x, y) \mathrm{d} y \stackrel{r \rightarrow 0}{\longrightarrow} 0 \quad \text { (see (3.2)). }
$$

By the Bouligand regularity theorem holding true in any $\mathfrak{S}^{*}$-harmonic space (see [9, Theorem 6.10.4]), the set $\Omega_{r}(x)$ is $\mathcal{L}$-regular for every $r>0$ and every $x \in \mathbb{R}^{N}$. Indeed, the function $y \mapsto \Gamma\left(x_{0}, y\right)-1 / r$ is an $\mathcal{H}$-barrier function (in the sense of [9, Definition 6.10.3]) at any point $x_{0}$ of $\partial \Omega_{r}(x)$.

To state our main theorem, we need the following notation and definitions about some average operators related to $\mathcal{L}$.

Definition 3.1 (Mean integral operators). Let $x \in \mathbb{R}^{N}$ and consider the functions, defined for $y \neq x$,

$$
\Gamma_{x}(y):=\Gamma(x, y), \quad \mathcal{K}_{x}(y):=\frac{\left\langle A(y) \nabla \Gamma_{x}(y), \nabla \Gamma_{x}(y)\right\rangle}{\left|\nabla \Gamma_{x}(y)\right|} .
$$

Let $\Omega \subseteq \mathbb{R}^{N}$ be an open set and suppose $u \in \operatorname{USC}(\Omega)$. For every fixed $\alpha>0$, and every $x \in \mathbb{R}^{N}$ and $r>0$ such that $\overline{\Omega_{r}(x)} \subset \Omega$, we introduce the following integrals:

$$
m_{r}(u)(x)=\int_{\partial \Omega_{r}(x)} u(y) \mathcal{K}_{x}(y) \mathrm{d} \sigma(y), \quad M_{r}(u)(x)=\frac{\alpha+1}{r^{\alpha+1}} \int_{0}^{r} \rho^{\alpha} m_{\rho}(u)(x) \mathrm{d} \rho,
$$

Furthermore, for every $x \in \mathbb{R}^{N}$ and every $r>0$, we set

$$
\begin{aligned}
& q_{r}(x)=\int_{\Omega_{r}(x)}\left(\Gamma_{x}(y)-\frac{1}{r}\right) \mathrm{d} y, \quad Q_{r}(x)=\frac{\alpha+1}{r^{\alpha+1}} \int_{0}^{r} \rho^{\alpha} q_{\rho}(x) \mathrm{d} \rho, \\
& \omega_{r}(x)=\frac{1}{\alpha r^{\alpha+1}} \int_{\Omega_{r}(x)}\left(r^{\alpha}-\Gamma_{x}^{-\alpha}(y)\right) \mathrm{d} y .
\end{aligned}
$$

Occasionally, the notation $M_{r}^{(\alpha)}, Q_{r}^{(\alpha)}, \omega_{r}^{(\alpha)}$ will also be applied. An alternative representation for $M_{r}(u)(x)$ is

$$
M_{r}(u)(x)=\frac{\alpha+1}{r^{\alpha+1}} \int_{\Omega_{r}(x)} u(y) K^{(\alpha)}(x, y) \mathrm{d} y,
$$

where we have set

$$
K^{(\alpha)}(x, y):=\frac{\left\langle A(y) \nabla \Gamma_{x}(y), \nabla \Gamma_{x}(y)\right\rangle}{\Gamma_{x}^{2+\alpha}(y)}
$$


There are also alternative formulas for $q_{r}(x), Q_{r}(x), \omega_{r}(x)$ (all proved in the Appendix), of independent interest: for every $x \in \mathbb{R}^{N}$ and every $\alpha, r>0$, one has

$$
\begin{aligned}
q_{r}(x) & =\int_{0}^{r} \frac{\left|\Omega_{s}(x)\right|}{s^{2}} \mathrm{~d} s, \\
Q_{r}(x) & =\int_{0}^{r} \frac{\left|\Omega_{s}(x)\right|}{s^{2}}\left(1-\left(\frac{s}{r}\right)^{\alpha+1}\right) \mathrm{d} s, \\
\omega_{r}(x) & =\frac{1}{r^{\alpha+1}} \int_{0}^{r} s^{\alpha-1}\left|\Omega_{s}(x)\right| \mathrm{d} s .
\end{aligned}
$$

Remark 3.2. The above definitions are correct. Indeed, note that $m_{r}(u)(x)$ is welldefined because $\partial \Omega_{r}(x)$ is a compact subset of $\Omega$ (see also hypothesis (G.2) on the fundamental solution), and $u$ is bounded from above on compact sets (since it is upper semicontinuous). We also remark that $q_{r}(x), Q_{r}(x), \omega_{r}(x)$ are strictly positive, for any $x \in \mathbb{R}^{N}$ and $r>0$. Also, they are finite since $\Gamma(x, \cdot)$ is locally summable.

Moreover, under the hypotheses of the above definition, we claim that the map $r \mapsto m_{r}(u)(x)$ is upper semicontinuous, so that $M_{r}(u)(x)$ is well-defined too. The claim follows from the following argument: Since $u \in \operatorname{USC}(\Omega)$ and $\partial \Omega_{r}(x)$ is compact, there exists a decreasing sequence $\left\{u_{j}\right\}_{j}$ of continuous functions on $\partial \Omega_{r}(x)$ converging pointwise to $u$; it is easily seen that $r \mapsto m_{r}\left(u_{j}\right)(x)$ is continuous (for every $j \in \mathbb{N}$ ) and that $m_{r}(u)(x)=\lim _{j \rightarrow \infty} m_{r}\left(u_{j}\right)(x)$. Hence $r \mapsto m_{r}(u)(x)$ is upper semicontinuous.

We shall prove in the Appendix that the above average operators do intervene, when $u$ is $C^{2}$, in remarkable mean value formulas generalizing the classical Gauss-Green formulas for Laplace's operator. These are recalled, for later use, in the following theorem.

Theorem 3.3 (Mean value formulas for $\mathcal{L}$ ). Let $m_{r}, M_{r}$ be the average operators in Definition 3.1. Let also $x \in \mathbb{R}^{N}$ and $r>0$. Then, for every function $u$ of class $C^{2}$ on an open set containing $\overline{\Omega_{r}(x)}$, we have the following $\mathcal{L}$-representation formulas:

$$
\begin{aligned}
& u(x)=m_{r}(u)(x)-\int_{\Omega_{r}(x)}\left(\Gamma(x, y)-\frac{1}{r}\right) \mathcal{L} u(y) \mathrm{d} y \\
& u(x)=M_{r}(u)(x)-\frac{\alpha+1}{r^{\alpha+1}} \int_{0}^{r} \rho^{\alpha}\left(\int_{\Omega_{\rho}(x)}\left(\Gamma(x, y)-\frac{1}{\rho}\right) \mathcal{L} u(y) \mathrm{d} y\right) \mathrm{d} \rho,
\end{aligned}
$$

We shall refer to (3.8) as the surface mean value formula for $\mathcal{L}$, whereas (3.9) will be called the solid mean value formula for $\mathcal{L}$.

For the proof of one of our main results (Theorem 4.2 in the next section), we need a representation formula also for the difference of the mean integral operators $m_{r}-M_{r}$ (involved in what we shall call the 'Reade-type condition'). This formula seems to be new in many contexts of interest (e.g., for sub-Laplacian operators). For instance, we shall prove the following result. 
Theorem 3.4. Let $x \in \mathbb{R}^{N}, r>0$ and $\alpha>0$. Then

$$
m_{r}(u)(x)-M_{r}^{(\alpha)}(u)(x)=\frac{1}{\alpha r^{\alpha+1}} \int_{\Omega_{r}(x)}\left(r^{\alpha}-\Gamma^{-\alpha}(x, y)\right) \mathcal{L} u(y) \mathrm{d} y
$$

for every $u \in C^{2}\left(\overline{\Omega_{r}(x)}, \mathbb{R}\right)$.

Theorems 3.3 and 3.4 will be proved in the Appendix, which also contains the proof of the following result, showing that $m_{r}, M_{r}, q_{r}, Q_{r}, \omega_{r}$ can be combined together to produce asymptotic definitions of $\mathcal{L} u$.

Proposition 3.5. With the notation of Definition 3.1, for every $x \in \mathbb{R}^{N}, R>0$ and $u \in C^{2}\left(\overline{\Omega_{R}(x)}, \mathbb{R}\right)$ we have

$$
\begin{aligned}
\lim _{r \rightarrow 0} \frac{m_{r}(u)(x)-u(x)}{q_{r}(x)} & =\lim _{r \rightarrow 0} \frac{M_{r}(u)(x)-u(x)}{Q_{r}(x)}=\lim _{r \rightarrow 0} \frac{m_{r}(u)(x)-M_{r}(u)(x)}{\omega_{r}(x)} \\
& =\mathcal{L} u(x) .
\end{aligned}
$$

Furthermore, the functions $(0, \infty) \ni r \mapsto q_{r}(x), Q_{r}(x)$ are increasing and

$$
\lim _{r \rightarrow 0} q_{r}(x)=\lim _{r \rightarrow 0} Q_{r}(x)=\lim _{r \rightarrow 0} \omega_{r}(x)=0 .
$$

Finally, if the function $s \mapsto\left|\Omega_{s}(x)\right| /$ s is increasing, then so is $r \mapsto \omega_{r}(x)$.

We next give the explicit values of our average operators in the classical case of the Laplace operator in $\mathbb{R}^{N}$.

Remark 3.6 (The average operators for $\mathcal{L}=\Delta$ ). Let $N \geq 3$ be fixed. In the classical case $A=$ identity matrix, i.e., $\mathcal{L}=\Delta$ is the Laplace operator in $\mathbb{R}^{N}$, we have $\Gamma(x, y)=c_{N}|y-x|^{2-N}$ for a suitable dimensional constant $c_{N}>0$. Actually, $c_{N}=\left(N(N-2) \omega_{N}\right)^{-1}$, where $\omega_{N}=|B(0,1)|$.

A direct computation gives $\mathcal{K}(x, y)=c_{N}(N-2)|y-x|^{1-N}$, which is constant on $\partial \Omega_{r}(x)$, so that (if $B(x, \rho)$ denotes the usual Euclidean ball of center $x$ and radius $\rho$ ) a simple computation produces

$$
m_{r}(u)(x)=\mathbf{S}_{\rho}(u)(x), \quad \text { where } \quad \rho=\left(c_{N} r\right)^{1 /(N-2)} .
$$

Here, $\mathbf{S}_{\rho}$ is the classical surface average operator which we introduced in (1.2). Note that $\sigma(\partial B(x, \rho))=\sigma_{N} \rho^{N-1}$, where $\sigma_{N}=\sigma(\partial B(0,1))=N \omega_{N}$, whence $\sigma_{N} c_{N}(N-2)=1$.

Concerning (3.6), we have $K^{(\alpha)}(x, y)=c_{N}^{-\alpha}(N-2)^{2}|y-x|^{\alpha(N-2)-2}$. This is constant iff we choose $\alpha=2 /(N-2)$. With this choice of $\alpha$, a simple computation gives

$$
M_{r}(u)(x)=\mathbf{B}_{\rho}(u)(x), \text { where } \rho=\left(c_{N} r\right)^{1 /(N-2)},
$$

where $\mathbf{B}_{\rho}$ is the classical solid average operator in (1.2). We next compute $q_{r}$ and $Q_{r}$ :

$$
\left\{\begin{array}{l}
q_{r}(x)=\frac{1}{2 N} \rho^{2} \\
Q_{r}(x)=\frac{\alpha+1}{2 N} \frac{N-2}{2+(\alpha+1)(N-2)} \rho^{2},
\end{array} \text { where } \rho=\left(c_{N} r\right)^{1 /(N-2)} .\right.
$$

The above choice $\alpha=2 /(N-2)$ produces $Q_{r}(x)=\frac{1}{2(N+2)} \rho^{2}$. 
Finally, we compute $\omega_{r}$. Since $\Omega_{s}(x)$ is the Euclidean ball of center $x$ and radius $\left(c_{N} s\right)^{1 /(N-2)}$, formula (11.30) in the Appendix gives

$$
\omega_{r}(x)=\frac{1}{N} \frac{1}{\alpha(N-2)+N} \rho^{2}, \quad \text { where } \rho=\left(c_{N} r\right)^{1 /(N-2)} .
$$

The normalizing choice $\alpha=2 /(N-2)$ produces $\omega_{r}(x)=\frac{1}{N(N+2)} \rho^{2}$.

\section{Mean value characterizations of $\mathcal{L}$-subharmonic functions: Main Theorem}

Before stating our main theorem, we need a last definition.

Definition 4.1 ( $m, M$-continuity). A function $u \in \operatorname{USC}(\Omega)$ defined on an open subset $\Omega$ of $\mathbb{R}^{N}$ will be called $m$-continuous in $\Omega$ if

$$
\lim _{r \rightarrow 0} m_{r}(u)(x)=u(x) \quad \text { for every } x \in \Omega \text {. }
$$

Analogously, $u$ is said to be $M$-continuous in $\Omega$ if $\lim _{r \rightarrow 0} M_{r}(u)(x)=u(x)$ for every $x \in \Omega$.

Notice that, from the very definition of $M_{r}$, it follows that $m$-continuity implies $M$-continuity. Also, we shall prove that any $u \in C(\Omega, \mathbb{R})$ is $m$-continuous (whence $M$-continuous). This is the reason why $m$ - and $M$-continuity assumptions do not explicitly appear in characterizations of continuous subharmonic functions (see, e.g., [14]).

We are ready to state the main result of this section.

Theorem 4.2. Let $\Omega$ be an open subset of $\mathbb{R}^{N}$ and let $u: \Omega \rightarrow[-\infty, \infty)$ be an upper semicontinuous function such that $\Omega(u)=\{x: u(x)>-\infty\}$ contains at least one point of every component of $\Omega$. Let $q_{r}, Q_{r}, \omega_{r}$ be as in Definition 3.1. Let also $R(x):=\sup \{r>$ $0: \Omega_{r}(x) \subseteq \Omega$ \}. Then, the following conditions are equivalent:

$(\underline{S})$ Subharmonicity: $u \in \underline{S}(\Omega)$ with respect to $\mathcal{L}$.

(1) $m$-submean condition: $u(x) \leq m_{r}(u)(x)$ for every $x \in \Omega$ and $r \in(0, R(x))$.

(2) $M$-submean condition: $u(x) \leq M_{r}(u)(x)$ for every $x \in \Omega$ and $r \in(0, R(x))$.

(3) Blaschke-type condition:

$$
\limsup _{r \rightarrow 0} \frac{m_{r}(u)(x)-u(x)}{q_{r}(x)} \geq 0 \quad \text { for every } x \in \Omega(u) .
$$

(4) Privaloff-type condition:

$$
\limsup _{r \rightarrow 0} \frac{M_{r}(u)(x)-u(x)}{Q_{r}(x)} \geq 0 \quad \text { for every } x \in \Omega(u) .
$$

(5) m-monotonicity: $u$ is m-continuous in $\Omega$, and $r \mapsto m_{r}(u)(x)$ is increasing on $(0, R(x))$ for every $x \in \Omega$.

(6) $M$-monotonicity: $u$ is $M$-continuous in $\Omega$, and $r \mapsto M_{r}(u)(x)$ is increasing on $(0, R(x))$ for every $x \in \Omega$. 
(7) Beckenbach-Radó-type condition: $u$ is m-continuous in $\Omega$, and

$$
M_{r}(u)(x) \leq m_{r}(u)(x)
$$

for every $x \in \Omega$ and every $r \in(0, R(x))$.

(8) Reade-type condition: $u$ is $m$-continuous in $\Omega$, and

$$
\liminf _{r \rightarrow 0} \frac{m_{r}(u)(x)-M_{r}(u)(x)}{\omega_{r}(x)} \geq 0 \quad \text { for every } x \in \Omega(u) .
$$

The proof of Theorem 4.2 is long and it will be divided into many steps. We shall prove the implications depicted in Table 1.

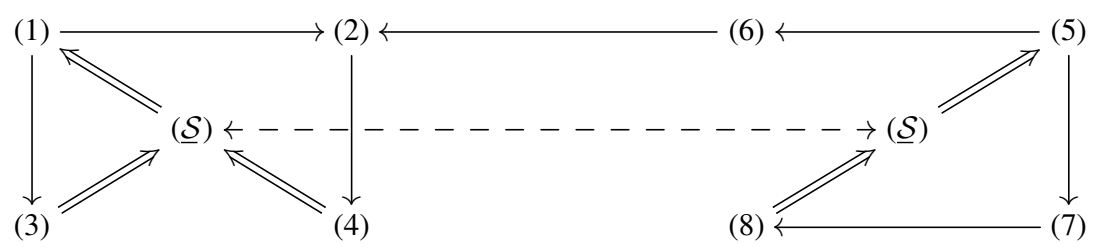

Table 1. Scheme of the proof of the main Theorem 4.2

In Table 1, the "single arrows" $\rightarrow$ denote implications which are straightforward consequences of the very definitions (whereas the dashed arrow is obviously tautological). The implications denoted with "double arrows" $\Rightarrow$ require more work, and they will be proved in separate sections (from 5.1 to 5.4).

\section{A Kozakiewicz-type theorem. Proof of Main Theorem}

The aim of this section is to prove Theorem 5.3 below. For the ordinary Laplace operator, this theorem goes back to a result due to W. Kozakiewicz [21]. Our effort here is to generalize it to the case of the second order operators considered in this paper.

We begin with some notation and definitions. Throughout this section, $\Omega$ will denote a fixed open subset of $\mathbb{R}^{N}$. We denote by $\mathcal{U}(\Omega)$ the set of functions $v: V \rightarrow[-\infty, \infty)$, defined on some open subset $V$ of $\Omega$, such that $v \in \operatorname{USC}(V)$ and $v>-\infty$ at some point of every component of $V$. We denote by $\mathcal{D}(v)$ (or $V(v)$, when the domain $V$ is specified) the set where $v$ takes on finite values. Finally, we denote by $\mathcal{F}(\Omega)$ the set of extended-real valued functions $f: A \rightarrow[-\infty, \infty]$, defined on some subset $A$ of $\Omega$.

Definition 5.1 ( $\mathcal{L}$-Kozakiewicz operator). With all the above notation, we say that a map

$$
G: \mathcal{U}(\Omega) \rightarrow \mathcal{F}(\Omega)
$$

is an $\mathcal{L}$-Kozakiewicz operator in $\Omega$ if it satisfies the following four axioms:

(K.1) If $v \in \mathcal{U}(\Omega)$ then $G(v)$ is defined on $\mathcal{D}(v)$, that is, $\mathcal{D}(G(v)) \supseteq \mathcal{D}(v)$.

(K.2) For every $h \in \mathcal{U}(\Omega)$ of class $C^{2}$ we have $G(h)=\mathcal{L} h$. 
(K.3) For every $v, h \in \mathcal{U}(\Omega)$, with $h$ of class $C^{2}$, defined on the same open set $V \subseteq \Omega$, we have $G(v+h)=G(v)+G(h)$.

(K.4) If $v \in \mathcal{U}(\Omega)$ and if $x_{0} \in \mathcal{D}(v)$ is a local maximum point of $v$, then $G(v)\left(x_{0}\right) \leq 0$.

Remark 5.2. We remark that, thanks to assumption (ND) on $\mathcal{L}$, for every bounded open set $U \subset \mathbb{R}^{N}$, there exists $w \in C^{2}(U, \mathbb{R})$ such that

$$
w>0 \text { and } \mathcal{L} w<0 \text { in } U .
$$

It suffices to take, if $i$ is as in hypothesis (ND), $w(x)=M-\exp \left(\lambda x_{i}\right)$, where

$$
\lambda>-\frac{\min _{\bar{U}} \sum_{j=1}^{N} \partial_{j} a_{j, i}}{\min _{\bar{U}} a_{i, i}} \text { and } \quad M>\max _{x \in \bar{U}} \exp \left(\lambda x_{i}\right) .
$$

We are ready to state and prove the following result.

Theorem 5.3 (of Kozakiewicz type, I). Let $\Omega \subseteq \mathbb{R}^{N}$ be an open set and let $G$ be an $\mathcal{L}$-Kozakiewicz operator in $\Omega$. Let $u: \Omega \rightarrow[-\infty, \infty)$ be an u.s.c. function, finite at some point of every component of $\Omega$, and such that

$$
G(u) \geq 0 \quad \text { in } \Omega(u) .
$$

Then $u$ is $\mathcal{L}$-subharmonic in $\Omega$.

Proof. Let $U$ be an $\mathcal{L}$-regular open set with $\bar{U} \subset \Omega$. We need to show that, for every $\varphi \in C(\partial U, \mathbb{R})$ with $u \leq \varphi$ on $\partial U$, we have $u \leq H_{\varphi}^{U}$ on $U$. Set $h:=H_{\varphi}^{U}$ and $v:=u-h$; we need to show that $v \leq 0$ on $U$.

Let $w \in C^{2}(U, \mathbb{R})$ be such that $w>0$ and $\mathcal{L} w<0$ on $U$ (see Remark 5.2). For every $\varepsilon>0$, set $v_{\varepsilon}:=u-h-\varepsilon w$. This function is defined on $U$ and finite on $\Omega(u) \cap U$. The compactness of $\bar{U}$ and the Heine-Borel covering lemma easily imply the existence of $x_{0} \in \bar{U}$ such that

$$
\sup _{V \cap U} v_{\varepsilon}=\sup _{U} v_{\varepsilon} \quad \text { for every } V \in \mathcal{N}_{x_{0}}
$$

where $\mathcal{N}_{x_{0}}$ denotes the set of open neighborhoods of $x_{0}$ (contained in $U$ ). We distinguish two cases: $x_{0} \in \partial U$ and $x_{0} \in U$.

If $x_{0} \in \partial U$, we have (notice that in this case $U=U \backslash\left\{x_{0}\right\}$ )

$$
\begin{gathered}
\sup _{U} v_{\varepsilon} \stackrel{(5.3)}{=} \inf _{V \in \mathcal{N}_{x_{0}}}\left(\sup _{V \cap U} v_{\varepsilon}\right)=\limsup _{U \ni x \rightarrow x_{0}} v_{\varepsilon}(x) \leq \limsup _{U \ni x \rightarrow x_{0}}(u(x)-h(x)) \\
\leq \limsup _{x \rightarrow x_{0}} u(x)-\liminf _{U \ni x \rightarrow x_{0}} h(x) \leq u\left(x_{0}\right)-\varphi\left(x_{0}\right) \leq 0 .
\end{gathered}
$$

Here, the first ' $\leq$ ' follows from $v_{\varepsilon}=u-h-\varepsilon w \leq u-h$ (see (5.1)), the second is obvious, the third follows from the upper semicontinuity of $u$ and the fact that

$$
\lim _{U \ni x \rightarrow x_{0}} H_{\varphi}^{U}(x)=\varphi\left(x_{0}\right) \quad\left(\text { as } x_{0} \in \partial U\right),
$$


whereas the last ' $\leq$ ' follows from $u \leq \varphi$ on $\partial U$ (and again $x_{0} \in \partial U$ ). Consequently, $\sup _{U} v_{\varepsilon} \leq 0$, that is, $u-h-\varepsilon w \leq 0$ on $U$. Letting $\varepsilon \rightarrow 0$, we infer $u-h \leq 0$ on $U$, which is what we aimed to prove.

If otherwise $x_{0} \in U$, then ( $v_{\varepsilon}$ being upper semicontinuous on $U$ )

$$
v_{\varepsilon}\left(x_{0}\right)=\max \left\{v_{\varepsilon}\left(x_{0}\right), \limsup _{x \rightarrow x_{0}} v_{\varepsilon}(x)\right\}=\inf _{V \in \mathcal{N}_{x_{0}}}\left(\sup _{V \cap U} v_{\varepsilon}\right) \stackrel{(5.3)}{=} \sup _{U} v_{\varepsilon},
$$

so that $x_{0}$ is a maximum point of $v_{\varepsilon}$ on $U$. If $v_{\varepsilon}\left(x_{0}\right)=-\infty$ then (since $x_{0}$ is a maximum point of $v_{\varepsilon}$ on $U$ ) $v_{\varepsilon} \equiv-\infty$ on $U$. This is possible iff $v \equiv-\infty$ on $U$, whence $v \leq 0$ on $U$, as we aimed to prove. We can therefore suppose that $x_{0} \in \mathcal{D}\left(v_{\varepsilon}\right)$. Since $v_{\varepsilon} \in \mathcal{U}(\Omega)$ and $G$ is an $\mathcal{L}$-Kozakiewicz operator, we have (by assumption (K.4))

$$
G\left(v_{\varepsilon}\right)\left(x_{0}\right) \leq 0 .
$$

On the other hand, since $x_{0} \in \mathcal{D}\left(v_{\varepsilon}\right) \subseteq \mathcal{D}(u)$, exploiting respectively conditions (K.3) and (K.2), the $\mathcal{L}$-harmonicity of $h$, hypothesis (5.2), and (5.1), we infer

$$
\begin{aligned}
G\left(v_{\varepsilon}\right)\left(x_{0}\right) & =G(u)\left(x_{0}\right)+G(-h-\varepsilon w)\left(x_{0}\right)=G(u)\left(x_{0}\right)-\mathcal{L} h\left(x_{0}\right)-\varepsilon \mathcal{L} w\left(x_{0}\right) \\
& =G(u)\left(x_{0}\right)-\varepsilon \mathcal{L} w\left(x_{0}\right) \geq-\varepsilon \mathcal{L} w\left(x_{0}\right)>0 .
\end{aligned}
$$

This contradicts (5.4). Hence $x_{0} \in U$ cannot hold, and the proof is complete by the previous case $x_{0} \in \partial U$.

Remark 5.4. A direct analysis of the proof of Theorem 5.3 shows that we exploited assumptions (K.3) and (K.4) only for a special class of functions $v$ in $\mathcal{U}(\Omega)$ : namely for functions of the type $v=u-\varphi$ (see the arguments in (5.5) and in (5.4)) where $\varphi$ is of class $C^{2}$ on some open subset of $\Omega$, and $u$ is the function whose $\mathcal{L}$-subharmonicity we aim to prove. This gives at once the following refinement of Theorem 5.3.

Theorem 5.5 (of Kozakiewicz type, II). Let $\Omega \subseteq \mathbb{R}^{N}$ be an open set and let $G$ : $\mathcal{U}(\Omega) \rightarrow \mathcal{F}(\Omega)$ be a map satisfying assumptions (K.1) and (K.2) in Definition 5.1. Let $u: \Omega \rightarrow[-\infty, \infty)$ be an u.s.c. function, finite at some point of every component of $\Omega$, and such that

$$
G(u) \geq 0 \text { in } \Omega(u) .
$$

Suppose finally that for every open set $U \subseteq \Omega$ and every $\varphi \in C^{2}(U, \mathbb{R})$ the following two conditions hold:

$(\mathrm{K} .3)^{\prime} \quad G(u+\varphi)=G(u)+G(\varphi)$;

(K.4) for every local maximum point $x_{0} \in \mathcal{D}(u)$ of $u-\varphi$, we have $G(u-\varphi)\left(x_{0}\right) \leq 0$.

Then $u$ is $\mathcal{L}$-subharmonic in $\Omega$.

We remark that the above hypothesis (K.4)' somewhat resembles the usual notion of viscosity subsolution.

With the preceding Kozakiewicz-type theorems at hand, we are ready for the proof of our main Theorem 4.2. We begin with the easiest part of the proof. 
Proof of the "single arrows" implications.

$(1) \rightarrow(2)$ : Let $x \in \Omega$ and $0<r<R(x)$. Then, by the very definition of $M_{r}$,

$$
M_{r}(u)(x)=\frac{\alpha+1}{r^{\alpha+1}} \int_{0}^{r} \rho^{\alpha} m_{\rho}(u)(x) \mathrm{d} \rho \geq \frac{\alpha+1}{r^{\alpha+1}} \int_{0}^{r} \rho^{\alpha} u(x) \mathrm{d} \rho=u(x) .
$$

$(1) \rightarrow(3)$ : This is trivial (recall that $q_{r}(x)>0$ ).

$(2) \rightarrow(4)$ : This is trivial (recall that $Q_{r}(x)>0$ ).

$(6) \rightarrow(2)$ : Towards a contradiction, suppose there exist $x \in \Omega$ and $\bar{r}>0$ such that $u(x)>$ $M_{\bar{r}}(u)(x)$. Then, if $u$ is $M$-continuous, we have

$$
M_{\rho}(u)(x)>M_{\bar{r}}(u)(x)
$$

for every $\rho>0$ small enough. In particular, the last inequality holds for some positive $\rho<\bar{r}$, contradicting the assumption that $r \mapsto M_{r}(u)(x)$ is increasing.

$(5) \rightarrow(6)$ : We already remarked that $m$-continuity implies $M$-continuity. Moreover, let $x \in \Omega$ and $0<r_{1}<r_{2}<R(x)$. If $M_{r_{1}}(u)(x)=-\infty$, then we obviously have $M_{r_{1}}(u)(x) \leq M_{r_{2}}(u)(x)$. Hence, we can assume that $M_{r_{1}}(u)(x)>-\infty$. This implies that the integral $\int_{0}^{r_{1}} \rho^{\alpha} m_{\rho}(u)(x) \mathrm{d} \rho$ is finite, so that $m_{\rho}(u)(x) \in \mathbb{R}$ for almost every $\rho \in\left(0, r_{1}\right)$. Consequently, due to the monotonicity assumption in condition (5) (jointly with $\left.r_{1}<r_{2}\right)$, the same is true of every $\rho \in\left(0, r_{2}\right]$ and

$$
-\infty<m_{\rho}(u)(x) \leq m_{r_{2}}(u)(x) \leq \frac{\sup }{\Omega_{r_{2}}(x)} u<\infty, \quad \forall \rho \in\left(0, r_{2}\right]
$$

(Recall that $u$ is u.s.c., and $\Omega_{r_{2}}(x)$ is bounded.) Dropping temporarily the " $(u)(x)$ " notation, by applying twice the monotonicity of $r \mapsto m_{r}$, it follows that

$$
\begin{aligned}
\frac{M_{r_{2}}}{\alpha+1} & =\frac{1}{r_{2}^{\alpha+1}}\left(\int_{0}^{r_{1}} \rho^{\alpha} m_{\rho} \mathrm{d} \rho+\int_{r_{1}}^{r_{2}} \rho^{\alpha} m_{\rho} \mathrm{d} \rho\right) \\
& =\underbrace{\left(\frac{1}{r_{2}^{\alpha+1}}-\frac{1}{r_{1}^{\alpha+1}}\right) \int_{0}^{r_{1}} \rho^{\alpha} \underbrace{m_{\rho}}_{\leq m_{r_{1}}} \mathrm{~d} \rho+\frac{M_{r_{1}}}{\alpha+1}+\frac{1}{r_{2}^{\alpha+1}} \int_{r_{1}}^{r_{2}} \rho^{\alpha} \underbrace{m_{\rho}}_{\geq m_{r_{1}}} \mathrm{~d} \rho}_{\leq 0} \\
& \geq \frac{M_{r_{1}}}{\alpha+1}+m_{r_{1}} \cdot\{\underbrace{\left\{\left(\frac{1}{r_{2}^{\alpha+1}}-\frac{1}{r_{1}^{\alpha+1}}\right) \int_{0}^{r_{1}} \rho^{\alpha} \mathrm{d} \rho+\frac{1}{r_{2}^{\alpha+1}} \int_{r_{1}}^{r_{2}} \rho^{\alpha} \mathrm{d} \rho\right\}}_{=0}=\frac{M_{r_{1}}}{\alpha+1} .
\end{aligned}
$$

This proves that $M_{r_{2}}(u)(x) \geq M_{r_{1}}(u)(x)$ and condition (6) follows.

Remark 5.6. The above computation proves that, if $f(\rho)$ is increasing function of $\rho>0$, then the same is true of the function $F(r):=\frac{\alpha+1}{r^{\alpha+1}} \int_{0}^{r} \rho^{\alpha} f(\rho) \mathrm{d} \rho$ (for any $\alpha>-1$ ). 
$(5) \rightarrow(7)$ : From the very definition of $M_{r}(u)(x)$ and the monotonicity of $\rho \mapsto m_{\rho}(u)(x)$, we immediately get

$$
M_{r}(u)(x)=\frac{\alpha+1}{r^{\alpha+1}} \int_{0}^{r} \rho^{\alpha} m_{\rho}(u)(x) \mathrm{d} \rho \leq \frac{\alpha+1}{r^{\alpha+1}} \int_{0}^{r} \rho^{\alpha} m_{r}(u)(x) \mathrm{d} \rho=m_{r}(u)(x)
$$

for every $x \in \Omega$ and $0<r<R(x)$.

$(7) \rightarrow(8)$ : Since $u$ is $m$-continuous, whence $M$-continuous, for every fixed $x \in \Omega(u)$ and for every sufficiently small $r>0, m_{r}(u)(x)$ and $M_{r}(u)(x)$ are finite. As a consequence, from the second part of condition (7), we have

$$
\frac{m_{r}(u)(x)-M_{r}(u)(x)}{\omega_{r}(x)} \geq 0
$$

for every small positive $r$ ( ince $\omega_{r}(x)>0$ ), so that the Reade-type condition (8) follows.

This completely proves the "single arrow" implications in Table 1.

We next turn to the "double arrows", to each of which we devote a separate subsection.

\subsection{Proof of $(3) \Rightarrow(\underline{\mathcal{S}})$ and $(4) \Rightarrow(\underline{\mathcal{S}})$}

Let $\Omega \subseteq \mathbb{R}^{N}$ be open and let $u: \Omega \rightarrow[-\infty, \infty)$ be an upper semicontinuous function, $u>-\infty$ in $\Omega(u)$, the latter set containing at least one point of every component of $\Omega$. Assume that Blaschke-type condition (4.1) holds. We have to prove that $u \in \underline{\mathcal{S}}(\Omega)$.

To this end, let $V$ be any open subset of $\Omega$, and let $v \in \operatorname{USC}(V)$ be finite at some point of every component of $V$ (we recall that, if these facts hold, we write $v \in \mathcal{U}(\Omega)$ ). We set

$$
G(v)(x):=\limsup _{r \rightarrow 0} \frac{m_{r}(v)(x)-v(x)}{q_{r}(x)}, \quad x \in \mathcal{D}(v) .
$$

We claim that $G$ is an $\mathcal{L}$-Kozakiewicz operator, according to Definition 5.1.

To begin with, axiom (K.1) is satisfied, by the very definition of $G$. Moreover, we know that $G(h)=\mathcal{L} h$ for every $C^{2}$ function $h$ on some open subset of $\Omega$ (see (3.11), Proposition 3.5). Hence axiom (K.2) holds. Furthermore, if $u, v \in \mathcal{U}(\Omega)$ have the same domain of finiteness, and $h$ is of class $C^{2}$, then, for every $x \in \mathcal{D}(v)=\mathcal{D}(v+h)$,

$$
G(v+h)(x)=G(v)(x)+G(h)(x) .
$$

This ensures that axiom (K.3) holds too. Finally, if $v \in \mathcal{U}(\Omega)$ and $x_{0} \in \mathcal{D}(v)$ is a local maximum point of $v$, we have $v\left(x_{0}\right)>-\infty$ and $v \leq v\left(x_{0}\right)$ in a neighborhood $U$ of $x_{0}$. This implies $m_{r}(v)\left(x_{0}\right) \leq v\left(x_{0}\right)$ whenever $\Omega_{r}\left(x_{0}\right) \subset U$, hence for every $r>0$ sufficiently small. This gives at once

$$
G(v)\left(x_{0}\right)=\limsup _{r \rightarrow 0} \frac{m_{r}(v)\left(x_{0}\right)-v\left(x_{0}\right)}{q_{r}\left(x_{0}\right)} \leq 0 .
$$

This ensures that axiom (K.4) holds true. 
Thus $G$ is an $\mathcal{L}$-Kozakiewicz operator. On the other hand, the Blaschke-type condition (4.1) ensures that $G(u) \geq 0$ on $\Omega(u)$. Our Kozakiewicz-type Theorem 5.3 implies that $u \in \underline{\mathcal{S}}(\Omega)$, as we aimed to prove.

The implication $(4) \Rightarrow(\underline{\mathcal{S}})$ can be proved in a straightforwardly analogous way, this time considering the operator

$$
G(v)(x):=\limsup _{r \rightarrow 0} \frac{M_{r}(v)(x)-v(x)}{Q_{r}(x)}
$$

defined for $v \in \mathcal{U}(\Omega)$ and $x \in \mathcal{D}(v)$ (here (3.11) in Proposition 3.5 is also required).

\subsection{Proof of $(\underline{\mathcal{S}}) \Rightarrow(1)$}

Theorem 5.7 ( $m$-submean property of $\underline{\mathcal{S}}(\Omega))$. Let $u \in \underline{\mathcal{S}}(\Omega)$ and suppose $\overline{\Omega_{r}(x)} \subset \Omega$. Then $u(x) \leq m_{r}(u)(x)$.

Proof. Let $u \in \mathcal{S}(\Omega)$ and suppose that $\overline{\Omega_{r}\left(x_{0}\right)} \subset \Omega$. Let also $\varphi: \partial \Omega_{r}\left(x_{0}\right) \rightarrow \mathbb{R}$ be a continuous function such that $\varphi \geq u$ on $\partial \Omega_{r}\left(x_{0}\right)$. Since $u \in \underline{\mathcal{S}}(\Omega)$ and $\Omega_{r}\left(x_{0}\right)$ is an $\mathcal{L}$-regular set, we have $u \leq h:=H_{\varphi}^{\bar{\Omega}_{r}\left(x_{0}\right)}$ on $\Omega_{r}\left(x_{0}\right)$. In particular, $u\left(x_{0}\right) \leq h\left(x_{0}\right)$. On the other hand, $h$ being an $\mathcal{L}$-harmonic function on $\Omega_{r}\left(x_{0}\right)$, for every $\rho \in(0, r)$ one has

$$
h\left(x_{0}\right) \stackrel{(3.8)}{=} m_{\rho}(h)\left(x_{0}\right)=\int_{\partial \Omega_{\rho}\left(x_{0}\right)} h(y) \mathcal{K}\left(x_{0}, y\right) \mathrm{d} \sigma(y) .
$$

Thus $u\left(x_{0}\right) \leq \int_{\partial \Omega_{\rho}\left(x_{0}\right)} h(y) \mathcal{K}\left(x_{0}, y\right) \mathrm{d} \sigma(y)$. Letting $\rho \rightarrow r-$, we get

$$
u\left(x_{0}\right) \leq \int_{\partial \Omega_{r}\left(x_{0}\right)} \varphi(y) \mathcal{K}\left(x_{0}, y\right) \mathrm{d} \sigma(y) .
$$

By taking the infimum with respect to the $\varphi$ 's in the set

$$
\mathfrak{F}:=\left\{\varphi \in C\left(\partial \Omega_{r}\left(x_{0}\right), \mathbb{R}\right): \varphi \geq\left. u\right|_{\partial \Omega_{r}\left(x_{0}\right)}\right\},
$$

we obtain (recall that $u$ is upper semicontinuous)

$$
u\left(x_{0}\right) \leq \inf _{\varphi \in \mathfrak{F}} \int_{\partial \Omega_{r}\left(x_{0}\right)} \varphi(y) \mathcal{K}\left(x_{0}, y\right) \mathrm{d} \sigma(y)=\int_{\partial \Omega_{r}\left(x_{0}\right)} u(y) \mathcal{K}\left(x_{0}, y\right) \mathrm{d} \sigma(y)
$$

Since the right-hand side is $m_{r}(u)\left(x_{0}\right)$, the proof is complete.

Theorem 5.7 above can be restated by saying that any $\mathcal{L}$-subharmonic function on $\Omega$ is also $m$-submean on $\Omega$, i.e., condition (1) of Theorem 4.2 holds. 


\subsection{Proof of $(\underline{\mathcal{S}}) \Rightarrow(5)$}

Let $\Omega \subseteq \mathbb{R}^{N}$ be open and let, throughout this section, $u \in \underline{\mathcal{S}}(\Omega)$ be fixed. We first remark that the $m$-submean property of $u$ (ensured by the implication $(\underline{\mathcal{S}}) \Rightarrow(1)$ previously proved) implies that $u$ is $m$-continuous (a fact interesting in its own right), whence

$$
u\left(x_{0}\right)=\lim _{r \rightarrow 0} m_{r}(u)\left(x_{0}\right) \quad \text { for every } u \in \underline{\mathcal{S}}(\Omega) \text { and every } x_{0} \in \Omega .
$$

Indeed, suppose first that $u\left(x_{0}\right)>-\infty$. Since $u \in \operatorname{USC}(\Omega)$, for every $\varepsilon>0$ there exists a neighborhood $V$ of $x_{0}$ such that $u(y)<u\left(x_{0}\right)+\varepsilon$ for all $y \in V$. Then, for every $r>0$ sufficiently small (recall that $\Omega_{r}\left(x_{0}\right) \downarrow\left\{x_{0}\right\}$ as $r \downarrow 0$ ),

$$
m_{r}(u)\left(x_{0}\right) \leq m_{r}\left(u\left(x_{0}\right)+\varepsilon\right)\left(x_{0}\right)=u\left(x_{0}\right)+\varepsilon .
$$

Keeping in mind the $m$-submean property of $u$, this implies

$$
m_{r}(u)\left(x_{0}\right)-\varepsilon \leq u\left(x_{0}\right) \leq m_{r}(u)\left(x_{0}\right) \leq m_{r}(u)\left(x_{0}\right)+\varepsilon
$$

for every $r>0$ small enough, which proves (5.8). We now prove (5.8) under the assumption that $u\left(x_{0}\right)=-\infty$. The upper semicontinuity of $u$ thus ensures that $\lim _{x \rightarrow x_{0}} u(x)=$ $-\infty$, so that, for every $N>0$, there exists a a neighborhood $V$ of $x_{0}$ such that $u(y)<-N$ for all $y \in V$. Arguing as above we get $m_{r}(u)\left(x_{0}\right)<-N$ for any $r>0$ small enough. This gives $\lim _{r \rightarrow 0} m_{r}(u)\left(x_{0}\right)=-\infty$, proving (5.8) again.

Note that (5.8) ensures that any $\mathcal{L}$-subharmonic function is $m$-continuous, so that the first part of condition (5) in Theorem 4.2 follows.

Lemma 5.8. For every $x_{0} \in \mathbb{R}^{N}$ and every $r>0$, we have

$$
\mathrm{d} \mu_{x_{0}}^{\Omega_{r}\left(x_{0}\right)}(y)=\mathcal{K}\left(x_{0}, y\right) \mathrm{d} \sigma(y),
$$

$\mathrm{d} \sigma$ denoting, as usual, the $(N-1)$-dimensional Hausdorff measure in $\mathbb{R}^{N}$.

Proof. Let $\varphi \in C\left(\partial \Omega_{r}\left(x_{0}\right), \mathbb{R}\right)$. Then the function

$$
\Omega_{r}\left(x_{0}\right) \ni x \mapsto h(x):=\int_{\partial \Omega_{r}\left(x_{0}\right)} \varphi(y) \mathrm{d} \mu_{x}^{\Omega_{r}\left(x_{0}\right)}(y)=H_{\varphi}^{\Omega_{r}\left(x_{0}\right)}(x)
$$

is $\mathcal{L}$-harmonic in $\Omega_{r}\left(x_{0}\right)$. As a consequence, if $0<\rho<r$ we also have

$$
h\left(x_{0}\right)=m_{\rho}(h)\left(x_{0}\right)=\int_{\partial \Omega_{\rho}\left(x_{0}\right)} h(y) \mathcal{K}\left(x_{0}, y\right) \mathrm{d} \sigma(y) .
$$

Now, since $\Omega_{r}\left(x_{0}\right)$ is $\mathcal{L}$-regular, $\lim _{y \rightarrow z} h(y)=\varphi(z)$ for every $z \in \partial \Omega_{r}\left(x_{0}\right)$. Therefore

$$
h\left(x_{0}\right) \stackrel{(5.11)}{=} \lim _{\rho \uparrow r} \int_{\partial \Omega_{\rho}\left(x_{0}\right)} h(y) \mathcal{K}\left(x_{0}, y\right) \mathrm{d} \sigma(y)=\int_{\partial \Omega_{r}\left(x_{0}\right)} \varphi(y) \mathcal{K}\left(x_{0}, y\right) \mathrm{d} \sigma(y) .
$$

This gives (if compared with the identity obtained by taking $x=x_{0}$ in (5.10))

$$
\int_{\partial \Omega_{r}\left(x_{0}\right)} \varphi(y) \mathrm{d} \mu_{x_{0}}^{\Omega_{r}\left(x_{0}\right)}(y)=\int_{\partial \Omega_{r}\left(x_{0}\right)} \varphi(y) \mathcal{K}\left(x_{0}, y\right) \mathrm{d} \sigma(y) .
$$

The arbitrariness of $\varphi \in C\left(\partial \Omega_{r}\left(x_{0}\right), \mathbb{R}\right)$ proves (5.9). 
The following proposition will prove the $m$-monotonicity condition (5) of Theorem 4.2.

Proposition 5.9. Let $u \in \underline{\mathcal{S}}(\Omega)$ and let $\overline{\Omega_{r}\left(x_{0}\right)} \subset \Omega$. Then

$$
0<\rho<r \Rightarrow m_{\rho}(u)\left(x_{0}\right) \leq m_{r}(u)\left(x_{0}\right) .
$$

Proof. With the notation in the assertion, let $\mathfrak{F}$ be as in (5.7) and take any $\varphi \in \mathfrak{F}$. Since $u \leq \varphi$ on $\partial \Omega_{r}\left(x_{0}\right)$, and $u \in \underline{\mathcal{S}}(\Omega)$, we infer

$$
u \leq h:=H_{\varphi}^{\Omega_{r}\left(x_{0}\right)} \quad \text { on } \Omega_{r}\left(x_{0}\right) .
$$

Hence $h$ is $\mathcal{L}$-harmonic in $\Omega_{r}\left(x_{0}\right)$, and for every $y \in \partial \Omega_{r}\left(x_{0}\right)$ we have $h(x) \rightarrow \varphi(y)$ as $x \rightarrow y$. As a consequence, for every $0<\rho<r$ we have

$$
\begin{aligned}
m_{\rho}(u)\left(x_{0}\right) & =\int_{\partial \Omega_{\rho}\left(x_{0}\right)} u(y) \mathcal{K}\left(x_{0}, y\right) \mathrm{d} \sigma(y) \stackrel{(5.13)}{\leq} \int_{\partial \Omega_{\rho}\left(x_{0}\right)} h(y) \mathcal{K}\left(x_{0}, y\right) \mathrm{d} \sigma(y) \\
& =m_{\rho}(h)\left(x_{0}\right)=h\left(x_{0}\right) \stackrel{(5.10)}{=} \int_{\partial \Omega_{r}\left(x_{0}\right)} \varphi(y) \mathrm{d} \mu_{x_{0}}^{\Omega_{r}\left(x_{0}\right)}(y) \\
& \stackrel{(5.9)}{=} \int_{\partial \Omega_{r}\left(x_{0}\right)} \varphi(y) \mathcal{K}\left(x_{0}, y\right) \mathrm{d} \sigma(y) .
\end{aligned}
$$

The infimum of the right-hand side over $\varphi \in \mathfrak{F}$ yields $\int_{\partial \Omega_{r}\left(x_{0}\right)} u(y) \mathcal{K}\left(x_{0}, y\right) \mathrm{d} \sigma(y)=$ $m_{r}(u)\left(x_{0}\right)$, and the proof of (5.12) is complete.

5.4. Proof of $(8) \Rightarrow(\underline{\mathcal{S}})$

We start with a real analysis lemma.

Lemma 5.10. Let $R>0$ and let $f, g:[0, R] \rightarrow \mathbb{R}$ satisfy

$$
\begin{aligned}
& f(0)=\lim _{r \rightarrow 0} f(r)=\max _{[0, R]} f, \\
& \inf _{[\varepsilon, R]} g>0 \quad \text { for every } \varepsilon \in(0, R) .
\end{aligned}
$$

Then there exists a strictly decreasing sequence $\left\{r_{k}\right\}_{k}$ in $(0, R]$ such that $r_{k} \rightarrow 0$ as $k \rightarrow \infty$ and, for every $k \in \mathbb{N}$,

$$
f(\rho) \geq f\left(r_{k}\right)-g\left(r_{k}\right) / k \quad \text { for } 0 \leq \rho \leq r_{k} .
$$

Proof. First of all, the conclusion is obvious if $f$ is constant on some interval of the form $[0, \varepsilon]$ with $\varepsilon>0$. Indeed, in this case (5.16) is satisfied, for example, by $r_{k}=$ $\min \{R / k, \varepsilon\}$ (notice that $g>0$ on $(0, R]$ thanks to $(5.15)$ ). It is then not restrictive to suppose that $f$ is not constant on any interval of the form $[0, \varepsilon]$ with $\varepsilon>0$. Furthermore, by shrinking $R$ if necessary, we may and do assume that $\inf _{[0, R]} f>-\infty$. We now construct the sequence $\left\{r_{k}\right\}_{k}$ inductively.

Let $R_{1} \in[0, R]$ be such that

$$
\begin{array}{cc}
\inf _{[0, R]} f=\inf _{V_{\varepsilon}} f & \text { for every } \varepsilon>0, \text { where } \\
V_{\varepsilon}:=\left(R_{1}-\varepsilon, R_{1}+\varepsilon\right) \cap[0, R] .
\end{array}
$$


We call $R_{1}$ a Weierstrass inf-point for $f$ on $[0, R]$. The existence of such $R_{1}$ easily follows from the compactness of $[0, R]$ and the Heine-Borel covering lemma. If $R_{1}=0$, from (5.14) and (5.17) we get

$$
\inf _{[0, R]} f \stackrel{(5.17)}{=} \sup _{\varepsilon>0}\left(\inf _{V_{\varepsilon}} f\right)=\min \left\{f(0), \liminf _{r \rightarrow 0} f(r)\right\} \stackrel{(5.14)}{=} \max _{[0, R]} f .
$$

Hence $f$ is constant, and-as we remarked-we may take $r_{1}=R$. We next construct $r_{1}$ supposing that $R_{1}>0$. In this case, by hypothesis (5.15) applied to $\varepsilon=R_{1} / 2>0$, there exists $r_{1} \in\left[R_{1} / 2, R\right]$ such that

$$
\inf _{\left[R_{1} / 2, R\right]} f>f\left(r_{1}\right)-\inf _{\left[R_{1} / 2, R\right]} g .
$$

This ensures that, with this choice of $r_{1}$, one has

$$
\inf _{[0, R]} f \stackrel{(5.17)}{=} \sup _{\varepsilon>0}\left(\inf _{V_{\varepsilon}} f\right) \geq \inf _{\left[R_{1} / 2, R\right]} f>f\left(r_{1}\right)-\inf _{\left[R_{1} / 2, R\right]} g \geq f\left(r_{1}\right)-g\left(r_{1}\right) .
$$

This implies, in particular, that $f(\rho) \geq f\left(r_{1}\right)-g\left(r_{1}\right)$ for $0 \leq \rho \leq r_{1}$, that is, (5.16) holds when $k=1$. We suppose we have constructed $r_{1}, \ldots, r_{k}$ satisfying (5.16) and such that

$$
0<r_{j+1} \leq r_{j} / 2
$$

for every $j=1, \ldots, k-1$. We next show how to obtain $r_{k+1}$ satisfying (5.16) and such that (5.18) holds for $j=k$. There exists a Weierstrass inf-point $R_{k+1}$ for $f$ on the compact interval [0, $\left.r_{k} / 2\right]$, that is, $R_{k+1} \in\left[0, r_{k} / 2\right]$ satisfies

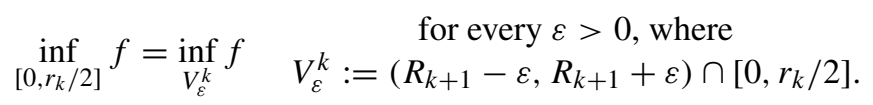

If $R_{k+1}=0$, from (5.19) and (5.14) we get

$$
\inf _{\left[0, r_{k} / 2\right]} f=\sup _{\varepsilon>0}\left(\inf _{V_{\varepsilon}^{k}} f\right)=\min \left\{f(0), \liminf _{r \rightarrow 0} f(r)\right\}=\max _{[0, R]} f \geq \max _{\left[0, r_{k} / 2\right]} f .
$$

This implies that $f$ is constant on $\left[0, r_{k} / 2\right]$, which contradicts our nonrestrictive assumptions on $f$. Hence $R_{k+1}>0$. In this case, by (5.15) we know that $\inf _{\left[R_{k+1} / 2, r_{k} / 2\right]} g \geq$ $\inf _{\left[R_{k+1} / 2, R\right]} g>0$. Hence, there exists $r_{k+1} \in\left[R_{k+1} / 2, r_{k} / 2\right]$ such that

$$
\inf _{\left[R_{k+1} / 2, r_{k} / 2\right]} f>f\left(r_{k+1}\right)-\frac{1}{k+1} \inf _{\left[R_{k+1} / 2, r_{k} / 2\right]} g .
$$

This ensures that the following chain of inequalities hold:

$$
\begin{aligned}
\inf _{\left[0, r_{k} / 2\right]} f & \stackrel{(5.19)}{=} \sup _{\varepsilon>0}\left(\inf _{V_{\varepsilon}^{k}} f\right) \geq \inf _{\left[R_{k+1} / 2, r_{k} / 2\right]} f \\
& \stackrel{(5.20)}{>} f\left(r_{k+1}\right)-\frac{1}{k+1} \inf _{\left[R_{k+1} / 2, r_{k} / 2\right]} g \geq f\left(r_{k+1}\right)-\frac{g\left(r_{k+1}\right)}{k+1} .
\end{aligned}
$$


To obtain the first ' $\geq$ ', we have taken $\varepsilon=R_{k+1} / 2$; the last ' $\geq$ ' follows from $r_{k+1} \in$ $\left[R_{k+1} / 2, r_{k} / 2\right]$. This implies that $f(\rho) \geq f\left(r_{k+1}\right)-\frac{g\left(r_{k+1}\right)}{k+1}$ for $0 \leq \rho \leq r_{k} / 2$, hence for $\rho \in\left[0, r_{k+1}\right]$ too.

Summarizing the above facts, we have inductively constructed a sequence $\left\{r_{k}\right\}_{k}$ in $(0, R]$ such that, for every $k \in \mathbb{N}$,

$$
0<r_{k+1} \leq r_{k} / 2, \quad \text { and } \quad f(\rho) \geq f\left(r_{k}\right)-g\left(r_{k}\right) / k, \quad \forall \rho \in\left[0, r_{k}\right]
$$

This completes the proof, since the first inequality implies that $r_{k} \downarrow 0$.

We are now ready to prove the implication $(8) \Rightarrow(\underline{\mathcal{S}})$, that is, the Reade-type condition implies $\mathcal{L}$-subharmonicity. We explicitly remark that this proof is more delicate than those involving Blaschke- and Privaloff-type conditions, in that we shall apply Theorem 5.5 instead of Theorem 5.3.

Let $\Omega \subseteq \mathbb{R}^{N}$ be open and $u \in \operatorname{USC}(\Omega)$ be finite at some point of every component of $\Omega$. We assume that $u$ is $m$-continuous and satisfies the Reade-type condition (4.3) in Theorem 4.2. Let also $V \subseteq \Omega$ be any open set and $v \in \operatorname{USC}(V)$ be finite at some point of every component of $V$. For any such $v$ we define $G(v)$ as follows:

$$
G(v): \mathcal{D}(v) \rightarrow[-\infty, \infty], \quad G(v)(x):=\liminf _{r \rightarrow 0} \frac{m_{r}(v)(x)-M_{r}(v)(x)}{\omega_{r}(x)} .
$$

(We agree to put $m_{r}(v)(x)-M_{r}(v)(x):=-\infty$ if $m_{r}(v)(x)=-\infty$.)

We aim to apply Theorem 5.5 for this operator $G$. First of all, axiom (K.1) holds from the definition of $G$. Next, from (11.29) in Remark 11.3, we know that, if $U \subseteq \Omega$ is any open set and if $h \in C^{2}(U, \mathbb{R})$, we have

$$
\lim _{r \rightarrow 0} \frac{m_{r}(h)(x)-M_{r}(h)(x)}{\omega_{r}(x)}=\mathcal{L} h(x), \quad x \in U .
$$

As a consequence, axiom (K.2) holds too. Moreover, if $v$ is as above, if $\varphi \in C^{2}(V, \mathbb{R})$ and $x \in \mathcal{D}(v)$, one has $G(v+h)(x)=G(v)(x)+G(h)(x)$. This ensures the validity of axiom (K.3), whence that of axiom (K.3)'.

Finally, suppose $x_{0} \in \mathcal{D}(u)$ is a local maximum point of $w:=u-\varphi$, where $\varphi \in$ $C^{2}(U, \mathbb{R})$ and $U$ is any open subset of $\Omega$. Note that $x_{0} \in \mathcal{D}(u)$ implies $w\left(x_{0}\right)>-\infty$. The assumption of $m$-continuity of $u$ jointly with the continuity of $\varphi$ ensure that $w$ is $m$-continuous too. Hence, there exists $R>0$ such that $\overline{\Omega_{R}\left(x_{0}\right)} \subset V$ and $-\infty<$ $m_{r}(w)\left(x_{0}\right) \leq w\left(x_{0}\right)$ for every $r \in(0, R]$. As a consequence, the function

$$
f:[0, R] \rightarrow \mathbb{R}, \quad f(r):= \begin{cases}m_{r}(w)\left(x_{0}\right) & \text { if } r \in(0, R], \\ w\left(x_{0}\right) & \text { if } r=0,\end{cases}
$$

satisfies the assumptions of Lemma 5.10. Also, the definition

$$
g(r):= \begin{cases}\omega_{r}\left(x_{0}\right) & \text { if } r \in(0, R] \\ 0 & \text { if } r=0,\end{cases}
$$


satisfies the relevant assumptions on the $g$-function of the same lemma. Indeed, the facts that $(0, \infty) \ni r \mapsto \omega_{r}\left(x_{0}\right)$ is continuous (see Remark 11.4) and strictly positive are enough to ensure the validity of (5.15).

Consequently, Lemma 5.10 ensures the existence of a decreasing sequence $\left\{r_{k}\right\}_{k}$ in $(0, R]$ such that $\lim _{k \rightarrow \infty} r_{k}=0$ and such that

$$
m_{\rho}(w)\left(x_{0}\right) \geq m_{r_{k}}(w)\left(x_{0}\right)-\omega_{r_{k}}\left(x_{0}\right) / k \quad \text { for every } \rho \in\left(0, r_{k}\right] \text { and } k \in \mathbb{N} .
$$

Then, from the very definition of $M_{r}$, we get

$$
M_{r_{k}}(w)\left(x_{0}\right) \geq m_{r_{k}}(w)\left(x_{0}\right)-\omega_{r_{k}}\left(x_{0}\right) / k \quad \text { for every } k \in \mathbb{N} .
$$

Therefore

$$
G(w)\left(x_{0}\right) \leq \liminf _{k \rightarrow \infty} \frac{m_{r_{k}}(w)\left(x_{0}\right)-M_{r_{k}}(w)\left(x_{0}\right)}{\omega_{r_{k}}\left(x_{0}\right)} \leq \lim _{k \rightarrow \infty} \frac{1}{k}=0 .
$$

This is axiom (K.4) for the function $w=u-\varphi$. This proves that we are in a position to apply Theorem 5.5 to the operator $G$. Since the Reade-type condition is precisely (5.6) in Theorem 5.5, we infer that $u$ is $\mathcal{L}$-subharmonic in $\Omega$, and the proof is complete.

\section{6. $\mathcal{L}$-subharmonicity of the average operators}

The aim of this section is to pave the way for the results of the following Sections 7 and 8 . To this end, we shall here establish a preliminary fact of interest in its own right: the mean integrals $m_{r}(u)(x), M_{r}(u)(x)$ of $u \in \mathcal{S}\left(\mathbb{R}^{N}\right)$, as functions of $x$, are themselves $\mathcal{L}$-subharmonic. To do this, we shall apply some arguments from [15], where mean integrals related to parabolic operators were concerned. Throughout this section, we shall use some Riesz-type representation theorems. For this reason, we shall need to add the hypothesis of $C^{\infty}$-hypoellipticity of $\mathcal{L}$.

Below, we assume that the entries of the matrix $A$ in (1.1) are $C^{\infty}$ functions on $\mathbb{R}^{N}$. Also, we make the following crucial assumption:

(HY) $\mathcal{L}$ is a $C^{\infty}$-hypoelliptic differential operator.

Hence, for every open set $\Omega \subseteq \mathbb{R}^{N}$, and for every $f \in C^{\infty}(\Omega, \mathbb{R})$, if $u \in L_{\mathrm{loc}}^{1}(\Omega)$ is a solution to $\mathcal{L} u=f$ in the sense of distributions, then $u$ coincides almost everywhere with a $C^{\infty}$ function on $\Omega$. As a consequence, $\mathcal{L}$-harmonic functions are $C^{\infty}$, whence, for example, $\Gamma(x, \cdot) \in C^{\infty}\left(\mathbb{R}^{N} \backslash\{x\}\right)$ for every fixed $x \in \mathbb{R}^{N}$.

Remark 6.1. Hypothesis (HY) brings along a plenty of consequences. We state some of them, to be applied in the following.

(i) Since $\mathcal{L}$ is self-adjoint, hypothesis (HY) ensures that the fundamental solution for $\mathcal{L}$ is symmetric, i.e., $\Gamma(x, y)=\Gamma(y, x)$ for every $x \neq y$ (see Bony [10, Section 6]).

(ii) The fundamental solution for $\mathcal{L}$ is unique. Indeed, if $\Gamma, \Gamma^{\prime}$ are two such fundamental solutions, then for every fixed $x \in \mathbb{R}^{N}$, the function $h:=\Gamma(x, \cdot)-\Gamma^{\prime}(x, \cdot)$ solves $\mathcal{L} h=0$ on $\mathbb{R}^{N}$ in the sense of distributions. Hence $h$ coincides with a smooth 
$\mathcal{L}$-harmonic function $\tilde{h}$ on $\mathbb{R}^{N}$, which (see axiom (G.2)) vanishes at infinity. The weak maximum principle easily implies that $\widetilde{h} \equiv 0$ on $\mathbb{R}^{N}$, that is (recall that the fundamental solution is smooth outside the diagonal), $\Gamma(x, y)=\Gamma^{\prime}(x, y)$ for every $y \in \mathbb{R}^{N} \backslash\{x\}$. By the symmetry result stated in (i) above, we infer that $\Gamma \equiv \Gamma^{\prime}$.

(iii) If $\Omega \subseteq \mathbb{R}^{N}$ is open and $u \in \mathcal{S}(\Omega)$, then, according to a result by Negrini and Scornazzani [26], $u \in L_{\mathrm{loc}}^{1}(\Omega)$ and there exists a Radon measure $\mu_{u}$ on $\Omega$ such that

$$
\int_{\Omega} u \mathcal{L} \varphi=\int_{\Omega} \varphi \mathrm{d} \mu_{u} \quad \text { for every } \varphi \in C_{0}^{\infty}(\Omega)
$$

(That is, $\mathcal{L} u=\mu_{u}$ in the sense of distributions.) We say that $\mu_{u}$ is the $\mathcal{L}$-Riesz measure of $u$ (on $\Omega$ ). Also, again by the results in [26], for every bounded open set $\Omega_{1}$ such that $\overline{\Omega_{1}} \subset \Omega$, there exists $h \in \mathcal{H}\left(\Omega_{1}\right)$ such that

$$
u(x)=h(x)-\int_{\overline{\Omega_{1}}} \Gamma(x, y) \mathrm{d} \mu_{u}(y)
$$

for almost every $x \in \Omega_{1}$. We shall soon show that this equality actually holds throughout $\Omega_{1}$ (see Remark 6.9).

For any Radon measure $\mu$ defined on an open set $\Omega \subseteq \mathbb{R}^{N}$, we set

$$
(\Gamma * \mu)(x):=\int_{\Omega} \Gamma(x, y) \mathrm{d} \mu(y), \quad x \in \mathbb{R}^{N} .
$$

This function, with values in $[0, \infty]$, is obviously lower semicontinuous in $\mathbb{R}^{N}$, thanks to Fatou's Lemma.

The following result will be useful.

Lemma 6.2. Let $\Omega \subseteq \mathbb{R}^{N}$ be open. Let $(\Lambda, \mathrm{d} \lambda)$ be a measure space and suppose that $\left\{u_{\ell}\right\}_{\ell \in \Lambda}$ is a family of $\mathcal{L}$-subharmonic functions in $\Omega$. Suppose furthermore that, for any $x \in \Omega$, the function $\ell \mapsto u_{\ell}(x)$ is $\mathrm{d} \lambda$-integrable on $\Lambda$ and set

$$
U: \Omega \rightarrow[-\infty, \infty), \quad U(x):=\int_{\Lambda} u_{\ell}(x) \mathrm{d} \lambda(\ell), \quad x \in \Omega .
$$

Suppose $U$ is finite at some point of every component of $\Omega$, and one of the following conditions hold:

1. $u_{\ell} \leq 0$ on $\Omega$ for every $\ell \in \Lambda$;

2. $\left\{u_{\ell}\right\}_{\ell \in \Lambda}$ is uniformly bounded from above and $\lambda(\Lambda)<\infty$.

Then $U$ is $\mathcal{L}$-subharmonic in $\Omega$.

Proof. Conditions 1 and 2 above yield the upper semicontinuity of $U$, as a simple application of Fatou's Lemma shows.

Suppose then that $U$ is finite at some point of every component of $\Omega$. To prove that $U \in \underline{\mathcal{S}}(\Omega)$, on account of the implication " $(2) \Rightarrow(\underline{\mathcal{S}})$ " in Theorem 4.2 , it suffices to prove 
that $U$ satisfies the $M$-submean condition on $\Omega$. Let $x \in \Omega$ and $r \in(0, R(x))$. Then Fubini-Tonelli's Theorem (see hypotheses 1 or 2 in the assertion) gives

$$
\begin{aligned}
M_{r}(U)(x) & =\int_{\Lambda}\left(\frac{\alpha+1}{r^{\alpha+1}} \int_{\Omega_{r}(x)} u_{\ell}(y) K^{(\alpha)}(x, y) \mathrm{d} y\right) \mathrm{d} \lambda(\ell) \\
& =\int_{\Lambda} M_{r}\left(u_{\ell}\right)(x) \mathrm{d} \lambda(\ell) \geq \int_{\Lambda} u_{\ell}(x) \mathrm{d} \lambda(\ell)=U(x) .
\end{aligned}
$$

In ' $\geq$ ' we used the hypothesis $u_{\ell} \in \underline{\mathcal{S}}(\Omega)$ for every $\ell \in \Lambda$, jointly with the implication "( $\underline{\mathcal{S}}) \Rightarrow(2)$ " of Theorem 4.2 .

Proposition 6.3. Let $\Omega \subseteq \mathbb{R}^{N}$ be open and suppose $\mu$ is a Radon measure on $\Omega$. Then the function $-\Gamma * \mu$ in $(6.2)$ belongs to $\underline{\mathcal{S}}\left(\mathbb{R}^{N}\right)$, provided it is not identically $-\infty$.

Proof. This follows from Lemma 6.2, applied to the nonpositive functions $\{-\Gamma(\cdot, y)\}_{y \in \Omega}$ and to the measure space $(\Omega, \mathrm{d} \mu)$. Note that $-\Gamma(\cdot, y) \in \underline{\mathcal{S}}\left(\mathbb{R}^{N}\right)$ for any $y \in \mathbb{R}^{N}$.

We next provide the following result, generalizing an analogous result proved in $[4$, Theorem 4.1], where sub-Laplacians are involved (see also [9, Theorem 9.3.10, p. 438]).

Theorem 6.4. Let $r>0$ and let $x, z \in \mathbb{R}^{N}$. Then

$$
m_{r}(\Gamma(\cdot, z))(x)=\min \{\Gamma(x, z), 1 / r\} .
$$

Proof. Let $z \in \mathbb{R}^{N}$ be fixed and set $w:=\Gamma(\cdot, z)$. We have to prove that

$$
m_{r}(w)(x)=\min \{w(x), 1 / r\} \quad \text { for every } x \in \mathbb{R}^{N} .
$$

We distinguish different cases.

(I) $z \notin \overline{\Omega_{r}(x)}$. In this case, in particular, $z \notin \Omega_{r}(x)$, that is (unraveling the definitions), $\min \{w(x), 1 / r\}=w(x)$. Hence (6.4) follows if we show that $m_{r}(w)(x)=w(x)$. But this is an immediate consequence of the mean value formula (3.8), jointly with the $\mathcal{L}$-harmonicity of $\Gamma(z, \cdot)=\Gamma(\cdot, z)=w$ in a neighborhood of $\overline{\Omega_{r}(x)}$.

(II) $z \in \Omega_{r}(x)$. This means $\min \{w(x), 1 / r\}=1 / r$, hence (6.4) follows if we show that $m_{r}(w)(x)=1 / r$. We need to distinguish two subcases:

If $z=x$, the following computation applies:

$$
m_{r}(w)(x)=\int_{\partial \Omega_{r}(x)} \Gamma(y, x) \mathcal{K}(x, y) \mathrm{d} \sigma(y)=\frac{1}{r} \int_{\partial \Omega_{r}(x)} \mathcal{K}(x, y) \mathrm{d} \sigma(y) \stackrel{(11.12)}{=} \frac{1}{r} .
$$

If $z \neq x$, there certainly exist two open Euclidean balls $B(z, \delta), B(z, \varepsilon)$ centered at $z$ and a smooth function $\psi_{\varepsilon}$ with the following properties:

$$
2 \varepsilon<\delta, \quad \overline{B(z, 2 \delta)} \subset \Omega_{r}(x), \quad x \notin B(z, 2 \delta),\left.\quad \psi_{\varepsilon}\right|_{B(z, \varepsilon)} \equiv 0,\left.\quad \psi_{\varepsilon}\right|_{\mathbb{R}^{N} \backslash B(z, 2 \varepsilon)} \equiv 1 .
$$


Then, since $\psi_{\varepsilon} \equiv 1$ on $\partial \Omega_{r}(x) \cup\{x\}$, and $w \psi_{\varepsilon} \in C^{\infty}\left(\mathbb{R}^{N}\right)$, we have

$$
\begin{aligned}
m_{r}(w)(x) & =m_{r}\left(w \psi_{\varepsilon}\right)(x) \stackrel{(3.8)}{=} w(x)+\int_{\Omega_{r}(x)}(\Gamma(x, y)-1 / r) \mathcal{L}\left(w \psi_{\varepsilon}\right)(y) \mathrm{d} y \\
& =w(x)+\int_{B(z, \delta)}(\Gamma(x, y)-1 / r) \mathcal{L}\left(w \psi_{\varepsilon}\right)(y) \mathrm{d} y .
\end{aligned}
$$

In the last equality, we used the fact that, since $\psi_{\varepsilon} \equiv 1$ outside $B(z, \delta)$ and $w$ is $\mathcal{L}$-harmonic outside $z$, we have $\mathcal{L}\left(w \psi_{\varepsilon}\right)=0$ on $\mathbb{R}^{N} \backslash B(z, \delta)$.

We claim that the last integral in (6.5) tends, as $\varepsilon \rightarrow 0$, to $1 / r-w(x)$, which will prove the desired equality $m_{r}(w)(x)=1 / r$. To prove the last claim, let us fix a function $\phi \in C_{0}^{\infty}\left(\mathbb{R}^{N}\right)$ such that

$$
\left.\phi\right|_{B(z, \delta)} \equiv 1,\left.\quad \phi\right|_{\mathbb{R}^{N} \backslash B(z, 2 \delta)} \equiv 0 .
$$

By this choice on $\phi$, we have

$$
\begin{aligned}
\int_{B(z, \delta)}(\Gamma(x, \cdot)- & 1 / r) \mathcal{L}\left(w \psi_{\varepsilon}\right)=\int_{\mathbb{R}^{N}}\{(\Gamma(x, \cdot)-1 / r) \phi\} \mathcal{L}\left(w \psi_{\varepsilon}\right) \\
& \text { (note that the function in braces is in } \left.C_{0}^{\infty}\left(\mathbb{R}^{N}\right) \text { since } x \notin B(z, 2 \delta)\right) \\
= & \int_{\mathbb{R}^{N}} \mathcal{L}\{(\Gamma(x, \cdot)-1 / r) \phi\} w \psi_{\varepsilon} \quad\left(\operatorname{set} \phi^{*}:=(\Gamma(x, \cdot)-1 / r) \phi\right) \\
= & \int_{\mathbb{R}^{N}}\left(\mathcal{L} \phi^{*}\right) w\left(\psi_{\varepsilon}-1\right)+\int_{\mathbb{R}^{N}}\left(\mathcal{L} \phi^{*}\right) w .
\end{aligned}
$$

Now, thanks to (2.1) (recalling that $w=\Gamma(\cdot, z)=\Gamma(z, \cdot)$ ), the last integral equals $-\phi^{*}(z)$, i.e., $-(\Gamma(x, z)-1 / r) \phi(z)=-w(x)+1 / r$. It remains to prove that $\int_{\mathbb{R}^{N}}\left(\mathcal{L} \phi^{*}\right) w\left(\psi_{\varepsilon}-1\right)$ vanishes as $\varepsilon \rightarrow 0$. This is a consequence of the following facts: $\mathcal{L} \phi^{*} \in C_{0}^{\infty}\left(\mathbb{R}^{N}\right)$ is independent of $\varepsilon, w=\Gamma(z, \cdot) \in L_{\text {loc }}^{1}\left(\mathbb{R}^{N}\right)$, and $\psi_{\varepsilon}-1$ is supported in $\overline{B(z, 2 \varepsilon)}$, which shrinks to $\{z\}$ as $\varepsilon \downarrow 0$.

(III) $z \in \partial \Omega_{r}(x)$. Consider the function

$$
y \mapsto u(y):=\Gamma(x, y)-\int_{\partial \Omega_{r}(x)} \Gamma(\eta, y) \mathrm{d} \mu_{x}^{\Omega_{r}(x)}(\eta) .
$$

We claim that $u$ is $\mathcal{L}$-subharmonic in $\mathbb{R}^{N} \backslash\{x\}$ as the sum of two $\mathcal{L}$-subharmonic functions. Indeed, $\Gamma(x, \cdot)$ is $\mathcal{L}$-harmonic in $\mathbb{R}^{N} \backslash\{x\}$, hence $\mathcal{L}$-subharmonic there; moreover, the $\mathcal{L}$-subharmonicity of $y \mapsto-\int_{\partial \Omega_{r}(x)} \Gamma(\eta, y) \mathrm{d} \mu_{x}^{\Omega_{r}(x)}(\eta)$ follows from an application of Lemma 6.2 to the family of $\mathcal{L}$-subharmonic functions $\{-\Gamma(\eta, \cdot)\}_{\eta \in \partial \Omega_{r}(x)}$ and to the measure space $\left(\partial \Omega_{r}(x), \mathrm{d} \mu_{x}^{\Omega_{r}(x)}\right)$. By Lemma 5.8,

$$
u(y)=\Gamma(x, y)-m_{r}(\Gamma(\cdot, y))(x) .
$$

Hence, by parts (I) and (II) proved above, we infer

$$
u=0 \quad \text { on } \mathbb{R}^{N} \backslash \Omega_{r}(x), \quad u=\Gamma(x, \cdot)-1 / r \quad \text { on } \Omega_{r}(x) .
$$


As $z \in \partial \Omega_{r}(x), u$ is $\mathcal{L}$-subharmonic in an open neighborhood of $z$. Since $\mathcal{L}$-subharmonic functions are $M$-continuous by Theorem 4.2, we have

$$
u(z)=\lim _{r \rightarrow 0} M_{r}(u)(z)
$$

Consider the function

$$
\mathbb{R}^{N} \ni y \mapsto u^{*}(y):= \begin{cases}0 & \text { if } y \in \mathbb{R}^{N} \backslash \Omega_{r}(x), \\ \Gamma(x, y)-1 / r & \text { if } y \in \Omega_{r}(x) .\end{cases}
$$

Note that $u^{*}$ is continuous in $\mathbb{R}^{N} \backslash\{x\}$ and that, by (6.7), $u=u^{*}$ on $\mathbb{R}^{N} \backslash \partial \Omega_{r}(x)$. Since $\partial \Omega_{r}(x)$ has vanishing Lebesgue measure, we have $u=u^{*}$ almost everywhere, whence

$$
u(z) \stackrel{(6.8)}{=} \lim _{r \rightarrow 0} M_{r}(u)(z)=\lim _{r \rightarrow 0} M_{r}\left(u^{*}\right)(z)=u^{*}(z)=0 .
$$

(In the second-last equality, we invoked the continuity of $u^{*}$.) By (6.6), $u(z)=0$ is equivalent to $\Gamma(x, z)=m_{r}(\Gamma(\cdot, z))(x)$, and the proof is complete.

Corollary 6.5. Let $r>0$ and let $x, z \in \mathbb{R}^{N}$. Then, for every $\alpha>0$,

$$
M_{r}(\Gamma(\cdot, z))(x)= \begin{cases}\frac{\alpha+1}{\alpha r} & \text { if } x=z, \\ \frac{1}{\alpha r}\left(\alpha+1-\frac{1}{(r \Gamma(x, z))^{\alpha}}\right) & \text { if } x \in \Omega_{r}(z), x \neq z, \\ \Gamma(x, z) & \text { if } x \notin \Omega_{r}(z) .\end{cases}
$$

Proof. A direct consequence of (6.3).

Corollary 6.6. Let $r>0$ and let $z \in \mathbb{R}^{N}$. Then the functions

$$
x \mapsto-m_{r}(\Gamma(\cdot, z))(x),-M_{r}(\Gamma(\cdot, z))(x)
$$

are continuous $\mathcal{L}$-subharmonic functions on $\mathbb{R}^{N}$. Also, they are bounded, they are left unchanged by interchanging $x$ and $z$, and the function $x \mapsto M_{r}(\Gamma(\cdot, z))(x)$ has a strict absolute minimum at $x=z$.

Proof. Let $r>0$ and let $z \in \mathbb{R}^{N}$ be fixed and set $u_{r}(x):=-m_{r}(\Gamma(\cdot, z))(x)$. By $(6.3)$, we have $u_{r}(x)=\max \{-\Gamma(x, z),-1 / r\}$. Hence $u \in \underline{\mathcal{S}}\left(\mathbb{R}^{N}\right)$ as the maximum of two $\mathcal{L}$ subharmonic functions in $\mathbb{R}^{N}$. The $\mathcal{L}$-subharmonicity of $x \mapsto U_{r}(x):=-M_{r}(\Gamma(\cdot, z))(x)$ now follows from the representation

$$
-M_{r}(\Gamma(\cdot, z))(x)=\frac{\alpha+1}{r^{\alpha+1}} \int_{0}^{r} \rho^{\alpha} u_{\rho}(x) \mathrm{d} \rho,
$$

which allows us to apply Lemma 6.2 to the family of nonpositive $\mathcal{L}$-subharmonic functions $\left\{u_{\rho}\right\}_{\rho \in[0, r]}$, and to the measure space $(\Lambda, \mathrm{d} \lambda)$, where $\Lambda=[0, r]$ and $\mathrm{d} \mu(\rho)=$ $\frac{\alpha+1}{r^{\alpha+1}} \rho^{\alpha} \mathrm{d} \rho$. Next, the continuity, boundedness and symmetry of the two functions in $(6.10)$ is obvious from (6.3) and (6.9) (recall that $\Gamma(x, z)=\infty$ if and only if $z=x$ ). Formula (6.9) also shows that $z$ is a strict minimum point for $U_{r}$. 
Corollary 6.7. Let $\Omega \subseteq \mathbb{R}^{N}$ be open. For every Radon measure $\mu$ on $\Omega$, and every $r>0$, each of the two functions $x \mapsto-m_{r}(\Gamma * \mu)(x),-M_{r}(\Gamma * \mu)(x)$ is $\mathcal{L}$-subharmonic in $\mathbb{R}^{N}$, provided it is not identically $-\infty$.

Proof. Fubini-Tonelli's Theorem readily furnishes the representations

$$
\begin{aligned}
& -m_{r}(\Gamma * \mu)(x)=\int_{\Omega} m_{r}(-\Gamma(\cdot, z))(x) \mathrm{d} \mu(z), \\
& -M_{r}(\Gamma * \mu)(x)=\int_{\Omega} M_{r}(-\Gamma(\cdot, z))(x) \mathrm{d} \mu(z) .
\end{aligned}
$$

The result stated in the corollary follows by applying Lemma 6.2 to the measure space $(\Omega, \mathrm{d} \mu)$ and the families of $\mathcal{L}$-subharmonic functions (see Corollary 6.6)

$$
\left\{x \mapsto m_{r}(-\Gamma(\cdot, z))(x)\right\}_{z \in \Omega}, \quad\left\{x \mapsto M_{r}(-\Gamma(\cdot, z))(x)\right\}_{z \in \Omega} .
$$

The above preliminary results will be used to prove the following theorem.

Theorem 6.8 ( $\mathcal{L}$-subharmonicity of the average operators). Let $u \in \mathcal{S}\left(\mathbb{R}^{N}\right)$ and $r>0$. Then the functions $x \mapsto m_{r}(u)(x), M_{r}(u)(x)$ are $\mathcal{L}$-subharmonic in $\mathbb{R}^{N}$, finite-valued and continuous.

Proof. Let $u \in \mathcal{S}\left(\mathbb{R}^{N}\right)$. From an obvious adaptation of the facts recalled in Remark 6.1(iii) (to provide a global representation on $\mathbb{R}^{N}$ ), we have

$$
u=h-\Gamma * \mu_{u} \quad \text { almost everywhere on } \mathbb{R}^{N},
$$

where $h$ is $\mathcal{L}$-harmonic in $\mathbb{R}^{N}$ and $\mu_{u}$ is the $\mathcal{L}$-Riesz measure of $u$. For any $r>0$, we infer from (6.11) (jointly with the $\mathcal{L}$-harmonicity of $h$ ) the full equality

$$
M_{r}(u)(x)=h(x)-M_{r}\left(\Gamma * \mu_{u}\right)(x) \quad \text { for every } x \in \mathbb{R}^{N} .
$$

From $M_{r}(u)(x) \geq u(x)$, we infer that $x \mapsto M_{r}(u)(x)$ is not identically $-\infty$, so that, by identity (6.12) itself, the same is true of the function $x \mapsto u_{r}(x):=-M_{r}\left(\Gamma * \mu_{u}\right)(x)$. An application of Corollary 6.7 thus proves the $\mathcal{L}$-subharmonicity of $u_{r}$ on $\mathbb{R}^{N}$. As $M_{r}(u)=$ $h+u_{r}$ (again by (6.12)), we infer $M_{r}(u) \in \underline{\mathcal{S}}\left(\mathbb{R}^{N}\right)$. Furthermore, we notice that (6.11) holds throughout, i.e.

$$
u=h-\Gamma * \mu_{u} \quad \text { on } \mathbb{R}^{N} .
$$

Indeed, since $\mathcal{L}$-subharmonic functions are $M$-continuous, letting $r \rightarrow 0$ in (6.12), we immediately get (6.13). By integration of both sides of (6.13), we infer

$$
m_{r}(u)(x)=h(x)-m_{r}\left(\Gamma * \mu_{u}\right)(x) \quad \text { for every } x \in \mathbb{R}^{N} .
$$

Starting from this identity and repeating the same argument as above, we can prove that $m_{r}(u) \in \underline{S}\left(\mathbb{R}^{N}\right)$. The fact that $m_{r}(u)(x)$ and $M_{r}(u)(x)$ are finite will be proved in Proposition 6.10 below, whereas their continuity will be proved in Remark 6.11. 
Remark 6.9. Let $u \in \underline{\mathcal{S}}(\Omega)$ and set, as usual, $\Omega(u)=\{x \in \Omega: u(x)>-\infty\}$. Let also $A$ be any bounded open set with $\bar{A} \subset \Omega$. Arguing as in the proof of Theorem 6.8, we can easily prove that there exists $h \in \mathcal{H}(A)$ such that

$$
u(x)=h(x)-\int_{\bar{A}} \Gamma(x, y) \mathrm{d} \mu_{u}(y) \quad \text { for every } x \in A .
$$

This shows that $u_{A}:=-\Gamma * \mu_{A}$ (here $\mu_{A}$ denotes $\left.\mu_{u}\right|_{\bar{A}}$, that is, the restriction to $\bar{A}$ of $\mu_{u}$ ) is finite precisely on the set $A \cap \Omega(u)$, hence on a dense subset of $A$ (see Remark 2.3). An application of Proposition 6.3 yields $u_{A} \in \underline{\mathcal{S}}(A)$.

As usual, we use the notation $R(x)=\sup \left\{r>0: \Omega_{r}(x) \subseteq \Omega\right\}$.

Proposition 6.10. Let $\Omega \subseteq \mathbb{R}^{N}$ be open and suppose that $u$ is $\mathcal{L}$-subharmonic in $\Omega$. Then the functions $(0, R(x)) \ni r \mapsto m_{r}(u)(x), M_{r}(u)(x)$ are continuous.

Proof. Let $u \in \underline{\mathcal{S}}(\Omega)$. Let also $\Omega_{1}$ be any open set with compact closure contained in $\Omega$. By Remark 6.1(iii), there exists $h_{1} \in \mathcal{H}\left(\Omega_{1}\right)$ such that

$$
u(x)=h_{1}(x)-\int_{\overline{\Omega_{1}}} \Gamma(x, y) \mathrm{d} \mu_{u}(y) \quad \text { for every } x \in \Omega_{1} .
$$

Suppose $x \in \Omega$ and let $r_{0} \in(0, R(x))$ be fixed. Let $\varepsilon>0$ be so small that $\overline{\Omega_{r}(x)} \subset \Omega$ for every $r \in\left(0, r_{0}+\varepsilon\right]$. Let also $\Omega_{1}=\Omega_{r_{0}+\varepsilon}(x)$. With this choice of $\Omega_{1}$, identity (6.15) holds, for some $\mathcal{L}$-harmonic function $h_{1}$ in $\Omega_{1}$. Then, by a direct integration of (6.15), by Fubini-Tonelli's Theorem and the $\mathcal{L}$-harmonicity of $h$, we infer, for any $r \in\left(0, r_{0}+\varepsilon\right)$,

$$
\begin{aligned}
m_{r}(u)(x) & =m_{r}\left(h_{1}\right)(x)-\int_{\overline{\Omega_{1}}}\left(\int_{\partial \Omega_{r}(x)} \Gamma(z, y) \mathcal{K}(x, z) \mathrm{d} \sigma(z)\right) \mathrm{d} \mu_{u}(y) \\
& =h_{1}(x)-\int_{\overline{\Omega_{1}}} m_{r}(\Gamma(\cdot, y))(x) \mathrm{d} \mu_{u}(y) .
\end{aligned}
$$

By applying identity (6.3) in Lemma 6.4, we get the representation

$$
m_{r}(u)(x)=h_{1}(x)-\int_{\overline{\Omega_{1}}} \min \{\Gamma(x, y), 1 / r\} \mathrm{d} \mu_{u}(y)
$$

for every $r \in\left(0, r_{0}+\varepsilon\right)$ (with $h_{1}$ depending on $u, \Omega, r_{0}, \varepsilon$, but independent of $r$ ). A simple dominated-convergence argument on (6.17) (note that $\mu_{u}\left(\overline{\Omega_{1}}\right)<\infty$ since $\overline{\Omega_{1}}$ is a compact subset of $\Omega$ and $\mu_{u}$ is a Radon measure on $\Omega$ ) proves the claimed continuity of $r \mapsto m_{r}(u)(x)$. The continuity of $r \mapsto M_{r}(u)(x)$ follows from that of $m_{r}(u)(x)$.

Remark 6.11. Let $\Omega \subseteq \mathbb{R}^{N}$ be open and let $u \in \underline{S}(\Omega)$. Let $x_{0} \in \Omega$. There certainly exist a bounded open neighborhood $U_{0}$ of $x_{0}$ and a positive $r_{0}$ both so small that $\overline{\Omega_{r}(x)} \subset \Omega$ for every $x \in \overline{U_{0}}$ and $r \in\left(0, r_{0}\right]$. Let also $\Omega_{1}$ be a bounded open subset of $\Omega$ such that

$$
\overline{\Omega_{r}(x)} \subset \Omega_{1} \subset \overline{\Omega_{1}} \subset \Omega \quad \text { for every } x \in \overline{U_{0}} \text { and every } r \in\left(0, r_{0}\right] .
$$


The set $\Omega_{1}$ depends only on $\Omega, x_{0}, r_{0}$. Arguing as in the previous proof, equality (6.15) holds for some $\mathcal{L}$-harmonic functions $h_{1}$ on $\Omega_{1}$. For any $x \in U_{0}$ and any $r \in\left(0, r_{0}\right)$, since $\partial \Omega_{r}(x)$ is contained in $\Omega_{1}$, we can take $m$-means on both sides of (6.15) to get, as in the computation in (6.16),

$$
m_{r}(u)(x)=h_{1}(x)-\int_{\overline{\Omega_{1}}} \min \{\Gamma(x, y), 1 / r\} \mathrm{d} \mu_{u}(y), \quad(x, r) \in U_{0} \times\left(0, r_{0}\right) .
$$

Note that the set $\overline{\Omega_{1}}$ is independent of $x \in U_{0}$ and $r \in\left(0, r_{0}\right)$. Since $h_{1}$ is $\mathcal{L}$-harmonic on $\Omega_{1}$, another dominated-convergence argument (recall that $\mu_{u}\left(\overline{\Omega_{1}}\right)<\infty$ since $\mu_{u}$ is Radon) suffices to show that the function $m_{r}(u)(x)$ is continuous in $(x, r)$ on $U_{0} \times\left(0, r_{0}\right)$. The same continuity property is thus inherited by $M_{r}(u)(x)$.

Gathering together the arguments in Remark 6.11 and in Theorem 6.8, it is not difficult to prove the following theorem. To state it, we need a notation: given a nonempty open set $\Omega \subseteq \mathbb{R}^{N}$ and $\varepsilon>0$ we set

$$
\Omega^{\varepsilon}:=\left\{x \in \Omega: \overline{\Omega_{\varepsilon}(x)} \subset \Omega\right\} .
$$

Note that, if $\varepsilon>0$ is small enough (depending on $\Omega$ ), the $\Omega^{\varepsilon}$ 's are nonempty open subsets of $\Omega$ such that, for every compact set $K \subset \Omega$, there exists a positive $\varepsilon_{0}=\varepsilon_{0}(K, \Omega)$ such that $K \subset \Omega^{\varepsilon}$ whenever $0<\varepsilon \leq \varepsilon_{0}$.

Theorem 6.12. Let $u \in \mathcal{S}(\Omega)$ and suppose $\varepsilon>0$ is small enough to ensure that $\Omega^{\varepsilon} \neq \emptyset$. Then, for every $r \in(0, \varepsilon)$, the functions $\Omega^{\varepsilon} \ni x \mapsto m_{r}(u)(x), M_{r}(u)(x)$ are $\mathcal{L}$-subharmonic, finite-valued and continuous in the open set $\Omega^{\varepsilon}$.

\section{Smoothing of $\mathcal{L}$-subharmonic functions}

The aim of this section is to prove a result on approximation of $\mathcal{L}$-subharmonic functions by smooth $\mathcal{L}$-subharmonic functions. A crucial tool is Theorem 6.12. The hypoellipticity hypothesis (HY) of the previous section is assumed.

Theorem 7.1 (Smooth approximation). Let $\Omega \subseteq \mathbb{R}^{N}$ be open and let $u \in \underline{\mathcal{S}}(\Omega)$. Let also $\varepsilon>0$ and consider the set $\Omega^{\varepsilon}$ introduced in (6.18). Then there exists a decreasing sequence $u_{n} \in \underline{\mathcal{S}}\left(\Omega^{\varepsilon}\right) \cap C^{\infty}\left(\Omega^{\varepsilon}, \mathbb{R}\right)$ converging pointwise to $u$ on $\Omega^{\varepsilon}$ as $n \rightarrow \infty$.

Proof. Let $\varepsilon>0$. Fix a $C^{\infty}$ function $\varphi: \mathbb{R} \rightarrow[0, \infty)$, compactly supported in the interval $(0,1)$, and such that $\int_{0}^{1} \varphi=1$. Note that $\varphi \equiv 0$ in a neighborhood of 0 . For any $r \in(0, \varepsilon)$, set

$$
F_{r}(u)(x):=\frac{1}{r} \int_{0}^{\infty} \varphi(t / r) m_{t}(u)(x) \mathrm{d} t, \quad x \in \Omega^{\varepsilon} .
$$

Note that $F_{r}(u)(x)$ is well-defined for every $x \in \Omega^{\varepsilon}$ since, in the above integral, the $t$ 's larger than $\varepsilon$ make the integrand vanish (as $r<\varepsilon$ ). We claim the following facts: 
(i) $F_{r}(u) \in C^{\infty}\left(\Omega^{\varepsilon}, \mathbb{R}\right)$ for any $r \in(0, \varepsilon)$.

(ii) $F_{r}(u) \in \underline{\mathcal{S}}\left(\Omega^{\varepsilon}\right)$ for any $r \in(0, \varepsilon)$.

(iii) If $0<r<s<\varepsilon$ then $F_{r}(u)(x) \leq F_{s}(u)(x)$, for every $x \in \Omega^{\varepsilon}$.

(iv) For every fixed $x \in \Omega^{\varepsilon}$, we have $F_{r}(u)(x) \rightarrow u(x)$ as $r \rightarrow 0$.

Once these properties have been proved, the theorem will follow by taking $u_{n}:=F_{1 / n}(u)$ with $n \in \mathbb{N}$ such that $n>1 / \varepsilon$. Let us now prove the above properties (i)-(iv).

(i) By taking into account the explicit expression of the surface mean integral (11.5) and of its kernel (11.6), we have the following computation (here $x \in \Omega^{\varepsilon}$ and $r \in(0, \varepsilon)$ ):

$$
\begin{aligned}
F_{r}(u)(x) & =\frac{1}{r} \int_{0}^{\infty} \varphi(t / r)\left(\int_{\Gamma_{x}=1 / t} u \frac{\left\langle A \nabla \Gamma_{x}, \nabla \Gamma_{x}\right\rangle}{\left|\nabla \Gamma_{x}\right|} \mathrm{d} \sigma\right) \mathrm{d} t \\
& \text { (use the change of variable } 1 / t=s) \\
& =\frac{1}{r} \int_{0}^{\infty} \varphi\left(\frac{1}{r s}\right)\left(\int_{\Gamma_{x}=s} u \frac{\left\langle A \nabla \Gamma_{x}, \nabla \Gamma_{x}\right\rangle}{\left|\nabla \Gamma_{x}\right|} \mathrm{d} \sigma\right) \frac{\mathrm{d} s}{s^{2}} \\
& =\frac{1}{r} \int_{0}^{\infty}\left(\int_{\Gamma_{x}=s} u \varphi\left(\frac{1}{r \Gamma_{x}}\right) \frac{\left\langle A \nabla \Gamma_{x}, \nabla \Gamma_{x}\right\rangle}{\Gamma_{x}^{2}} \frac{\mathrm{d} \sigma}{\left|\nabla \Gamma_{x}\right|}\right) \mathrm{d} s
\end{aligned}
$$

(by Federer's coarea formula)

$$
=\frac{1}{r} \int_{\mathbb{R}^{N}} u(y) \varphi\left(\frac{1}{r \Gamma(x, y)}\right) \frac{\left\langle A(y) \nabla \Gamma_{x}(y), \nabla \Gamma_{x}(y)\right\rangle}{\Gamma(x, y)^{2}} \mathrm{~d} y .
$$

This evidently shows that $F_{r}(u) \in C^{\infty}\left(\Omega^{\varepsilon}, \mathbb{R}\right)$ for any $r \in(0, \varepsilon)$ : Indeed, since $\varphi \equiv 0$ near 0 , the last integral extends over $\Omega_{r a}(x) \backslash \Omega_{r b}(x)$ (with $0<b<a<\infty$ ), a set where $\Gamma_{x}$ is smooth and bounded.

(ii) The $\mathcal{L}$-subharmonicity of $F_{r}(u)$ follows from Lemma 6.2, by considering the measure space $(\Lambda, \mathrm{d} \lambda)$, where $\Lambda=(0, \infty), \mathrm{d} \lambda(t)=(1 / r) \varphi(t / r) \mathrm{d} t$, and the family of functions $\left\{x \mapsto m_{t}(u)(x)\right\}_{t \in(0, \varepsilon)}$. Indeed, for any fixed $t \in(0, \varepsilon)$, the map $x \mapsto m_{t}(u)(x)$ is $\mathcal{L}$-subharmonic in $\Omega^{\varepsilon}$ : this follows from Theorem 6.12.

(iii) Let $x \in \Omega^{\varepsilon}$ be fixed. Since $u \in \underline{\mathcal{S}}(\Omega)$, from condition (5) of Theorem 4.2 it follows that $t \mapsto m_{t}(u)(x)$ is increasing on $(0, \varepsilon)$. The monotonicity of $r \mapsto F_{r}(u)(x)$ now follows by arguing as in Remark 5.6 (also using the fact that $(1 / r) \int_{0}^{\infty} \varphi(t / r) \mathrm{d} t=1$ for every $r>0$ ).

(iv) Thanks to our assumptions on the cut-off function $\varphi$, we have

$$
\left|F_{r}(u)(x)-u(x)\right| \leq \frac{1}{r} \int_{0}^{r} \varphi(t / r)\left|m_{t}(u)(x)-u(x)\right| \mathrm{d} t .
$$

The assertion in (iv) then follows immediately from the $m$-continuity of $\mathcal{L}$-subharmonic functions (see (5.8)).

\section{8. $\mathcal{L}$-subharmonic functions in the sense of distributions}

The aim of this section is to provide a further characterization of functions in $\underline{\mathcal{S}}(\Omega)$ as weak solutions to $\mathcal{L} u \geq 0$. The hypoellipticity hypothesis (HY) of Section 6 is assumed. 
Theorem 8.1. Let $\Omega \subseteq \mathbb{R}^{N}$ be an open set and let $u: \Omega \rightarrow[-\infty, \infty)$ be upper semicontinuous. Then $u \in \mathcal{S}(\Omega)$ if and only if the following facts hold: $u$ is M-continuous in $\Omega, u \in L_{\mathrm{loc}}^{1}(\Omega)$ and $\mathcal{L} u \geq 0$ in the sense of distributions.

Proof. We begin with the 'only if' part. Suppose $u \in \underline{\mathcal{S}}(\Omega)$. The $M$-continuity of $u$ is ensured by (5.8) (and the fact that $m$-continuity implies $M$-continuity). Moreover, by the results contained in [26], it follows that $u \in L_{\text {loc }}^{1}(\Omega)$ and that (arguing as in the proof of Theorem 6.8), for every bounded open set $\Omega^{\prime} \subset \Omega, u=h-\Gamma * \mu$ on $\Omega^{\prime}$, where $h$ is $\mathcal{L}$-harmonic in $\Omega^{\prime}$ and $\mu$ is a Radon measure on $\Omega^{\prime}$. This decomposition of $u$ easily implies that $\mathcal{L} u \geq 0$ in the sense of distributions: indeed, if $\varphi \in C_{0}^{\infty}(\Omega), \varphi \geq 0$ and $\Omega^{\prime}$ contains the support of $\varphi$, one has (see also (2.1))

$$
\begin{aligned}
\int_{\Omega} u \mathcal{L} \varphi & =\int_{\Omega^{\prime}} h \mathcal{L} \varphi-\int_{\Omega^{\prime}}(\Gamma * \mu)(x) \mathcal{L} \varphi(x) \mathrm{d} x \\
& =-\int_{\Omega}\left(\int_{\Omega^{\prime}} \Gamma(x, y) \mathcal{L} \varphi(x) \mathrm{d} x\right) \mathrm{d} \mu(y)=\int_{\Omega} \varphi(y) \mathrm{d} \mu(y) \geq 0 .
\end{aligned}
$$

We next turn to the 'if' part. Suppose that $u \in \operatorname{USC}(\Omega)$ is $M$-continuous in $\Omega, u \in$ $L_{\text {loc }}^{1}(\Omega)$ and $\mathcal{L} u \geq 0$ in the weak sense of distributions. The $L_{\text {loc }}^{1}$ hypothesis ensures that $u$ is finite on a dense subset of $\Omega$.

In order to prove that $u \in \mathcal{S}(\Omega)$, we verify that $(0, R(x)) \ni r \mapsto M_{r}(u)(x)$ is increasing, for every $x \in \Omega$ (and then we apply the characterization in condition (6) of our Theorem 4.2). To this end, fix $x_{0} \in \Omega$ and consider, as usual, $R\left(x_{0}\right)=\sup \{r>0$ : $\Omega_{r}\left(x_{0}\right) \subseteq \Omega$ \}. Since $\Omega$ is open, we have $R\left(x_{0}\right)>0$ (see also (3.2)). Let $\varepsilon>0$ be small, with $\varepsilon \ll R\left(x_{0}\right) / 3$. Let also $\varphi \in C^{\infty}([0, \infty), \mathbb{R})$ be nonnegative and such that $\varphi$ is constant in $[0, \varepsilon]$ and $\varphi \equiv 0$ in $\left[R\left(x_{0}\right)-\varepsilon, \infty\right]$.

Then we consider the function

$$
\phi(x):=\varphi\left(\frac{1}{\Gamma\left(x_{0}, x\right)}\right), \quad x \in \mathbb{R}^{N},
$$

with the obvious convention that $\phi\left(x_{0}\right)=\varphi(0)$. With this choice of $\varphi$ and thanks to our assumptions on $\Gamma$ and on $\mathcal{L}$ see (G.2) and (HY) we find that $\phi \in C_{0}^{\infty}\left(\mathbb{R}^{N}\right), \phi$ is constant in a neighborhood of $x_{0}$ (on $\Omega_{\varepsilon}\left(x_{0}\right)$, for instance) and $\phi \equiv 0$ outside a larger neighborhood of $x_{0}$, indeed $\phi \equiv 0$ on $\mathbb{R}^{N} \backslash \Omega_{R\left(x_{0}\right)-\varepsilon}\left(x_{0}\right)$. By definition of $R\left(x_{0}\right)$, this last fact ensures that $\phi \in C_{0}^{\infty}(\Omega)$. Since also $\phi \geq 0$, the hypothesis that $\mathcal{L} u \geq 0$ in the sense of distributions gives

$$
\int_{\Omega} u(x) \mathcal{L} \phi(x) \mathrm{d} x \geq 0 .
$$

Now, a direct computation of $\mathcal{L} \phi$ shows that

$$
\begin{aligned}
\mathcal{L} \phi & =\operatorname{div}(A \nabla \phi)=-\operatorname{div}\left(\varphi^{\prime}\left(\Gamma_{x_{0}}^{-1}\right) \Gamma_{x_{0}}^{-2} A \nabla \Gamma_{x_{0}}\right) \\
& =-\varphi^{\prime}\left(\Gamma_{x_{0}}^{-1}\right) \Gamma_{x_{0}}^{-2} \operatorname{div}\left(A \nabla \Gamma_{x_{0}}\right)-\left\langle\nabla\left(\varphi^{\prime}\left(\Gamma_{x_{0}}^{-1}\right) \Gamma_{x_{0}}^{-2}\right), A \nabla \Gamma_{x_{0}}\right\rangle .
\end{aligned}
$$


The first summand vanishes since $\operatorname{div}\left(A \nabla \Gamma_{x_{0}}\right)=\mathcal{L}\left(\Gamma\left(x_{0}, \cdot\right)\right)=0$; the second equals

$$
\begin{aligned}
\left\langle\varphi^{\prime \prime}\left(\Gamma_{x_{0}}^{-1}\right) \Gamma_{x_{0}}^{-4} \nabla \Gamma_{x_{0}}, A \nabla \Gamma_{x_{0}}\right\rangle & +\left\langle 2 \varphi^{\prime}\left(\Gamma_{x_{0}}^{-1}\right) \Gamma_{x_{0}}^{-3} \nabla \Gamma_{x_{0}}, A \nabla \Gamma_{x_{0}}\right\rangle \\
& =\left\langle\nabla \Gamma_{x_{0}}, A \nabla \Gamma_{x_{0}}\right\rangle \Gamma_{x_{0}}^{-4} \cdot\left\{\varphi^{\prime \prime}\left(\Gamma_{x_{0}}^{-1}\right)+2 \Gamma_{x_{0}} \varphi^{\prime}\left(\Gamma_{x_{0}}^{-1}\right)\right\} .
\end{aligned}
$$

We have thus proved that

$$
\mathcal{L} \phi=\left\langle\nabla \Gamma_{x_{0}}, A \nabla \Gamma_{x_{0}}\right\rangle \Gamma_{x_{0}}^{-4} \cdot\left\{\varphi^{\prime \prime}\left(\Gamma_{x_{0}}^{-1}\right)+2 \Gamma_{x_{0}} \varphi^{\prime}\left(\Gamma_{x_{0}}^{-1}\right)\right\} .
$$

Consequently, an application of Federer's coarea formula gives

$$
\begin{aligned}
0 & \stackrel{(8.1)}{\leq} \int_{\Omega} u \mathcal{L} \phi=\int_{\varepsilon}^{R\left(x_{0}\right)-\varepsilon}\left(\int_{1 / \Gamma_{x_{0}}=t} u \mathcal{L} \phi \frac{\mathrm{d} \sigma}{\left|\nabla\left(1 / \Gamma_{x_{0}}\right)\right|}\right) \mathrm{d} t \\
& =\int_{0}^{\infty}\left(\int_{1 / \Gamma_{x_{0}}=t} u \Gamma_{x_{0}}^{2} \mathcal{L} \phi \frac{\mathrm{d} \sigma}{\left|\nabla \Gamma_{x_{0}}\right|}\right) \mathrm{d} t \quad(\text { see (8.2) and (11.6)) } \\
& =\int_{0}^{\infty}\left(\int_{1 / \Gamma_{x_{0}}=t} u \Gamma_{x_{0}}^{-2} \mathcal{K}\left(x_{0}, \cdot\right)\left\{\varphi^{\prime \prime}\left(\Gamma_{x_{0}}^{-1}\right)+2 \Gamma_{x_{0}} \varphi^{\prime}\left(\Gamma_{x_{0}}^{-1}\right)\right\} \mathrm{d} \sigma\right) \mathrm{d} t \\
& =\int_{0}^{\infty} t^{2}\left\{\varphi^{\prime \prime}(t)+2 t^{-1} \varphi^{\prime}(t)\right\}\left(\int_{1 / \Gamma_{x_{0}}=t} u \mathcal{K}\left(x_{0}, \cdot\right) \mathrm{d} \sigma\right) \mathrm{d} t \\
& =\int_{0}^{\infty}\left\{t^{2} \varphi^{\prime \prime}(t)+2 t \varphi^{\prime}(t)\right\} m_{t}(u)\left(x_{0}\right) \mathrm{d} t=\int_{0}^{\infty}\left(t^{2} \varphi^{\prime}\right)^{\prime} m_{t}(u)\left(x_{0}\right) \mathrm{d} t .
\end{aligned}
$$

We have thus proved that

$$
\int_{0}^{\infty}\left(t^{2} \varphi^{\prime}\right)^{\prime} m_{t}(u)\left(x_{0}\right) \mathrm{d} t \geq 0 .
$$

Let now $h \in C_{0}^{\infty}$ be nonnegative and suppose the support of $h$ is a compact subset of $\left(0, R\left(x_{0}\right)\right)$, say $\left[h_{1}, h_{2}\right]$. The definition

$$
\varphi(t):=\int_{t}^{\infty} \frac{h(s)}{s^{2}} \mathrm{~d} s, \quad t \in[0, \infty),
$$

produces a function satisfying the requisites of the previous computations (take, for instance, $\left.\varepsilon<\min \left\{R\left(x_{0}\right) / 3, h_{1}, R\left(x_{0}\right)-h_{2}\right\}\right)$. Inserting this $\varphi$ in (8.3), we obtain

$$
\int_{0}^{\infty} h^{\prime}(t) m_{t}(u)\left(x_{0}\right) \mathrm{d} t \leq 0, \quad \forall h \in C_{0}^{\infty}\left(\left(0, R\left(x_{0}\right)\right), \mathbb{R}\right), h \geq 0 .
$$

By Lemma 8.2.13 of [9, p. 405], this ensures that the map defined by

$$
r \mapsto \frac{\alpha+1}{r^{\alpha+1}} \int_{0}^{r} \rho^{\alpha} m_{\rho}(u)\left(x_{0}\right) \mathrm{d} \rho=M_{r}(u)\left(x_{0}\right)
$$

is increasing on $\left(0, R\left(x_{0}\right)\right)$. The proof is complete. 


\section{The case of sub-Laplacians: Improved results}

The aim of this section is to apply the results of the previous sections to the case of subLaplacians on Carnot groups. This will allow us to improve former results contained in [9] and [5, 6]. In this context, it seems more natural to rewrite our mean integral operators $m_{r}, M_{r}$ in terms of gauge balls (definitions can be found below). We shall see that the operators $q_{r}, Q_{r}, \omega_{r}$ turn out to have a particularly simple form. Furthermore, we shall have occasion to prove a well-behaved property (left-invariance) of $m_{r}(u)(x)$ and $M_{r}(u)(x)$.

Recalls on Carnot groups and sub-Laplacians. We begin by recalling some basic facts concerning sub-Laplacians and Carnot groups. All the details can be found in [9, Chapter 1]. A Carnot group is a connected and simply connected (real) Lie group $(G, \cdot)$ whose Lie algebra $\operatorname{Lie}(G)$ admits a stratification, i.e., a decomposition $\operatorname{Lie}(G)=V_{1} \oplus \cdots \oplus V_{r}$, where

$$
V_{2}=\left[V_{1}, V_{1}\right], V_{3}=\left[V_{1}, V_{2}\right], \ldots, V_{r}=\left[V_{1}, V_{r-1}\right] \quad \text { and } \quad\left[V_{1}, V_{r}\right]=\{0\} .
$$

Thus $G$ is nilpotent of step $r$, whence $\operatorname{Exp}: \operatorname{Lie}(G) \rightarrow G$ is a (global) smooth diffeomorphism, with inverse $\log : G \rightarrow \operatorname{Lie}(G)$. We fix a basis $\mathcal{Z}=\left\{Z_{1}, \ldots, Z_{N}\right\}$ of $\operatorname{Lie}(G)$ called adapted to the stratification, that is,

$$
V_{1}=\operatorname{span}\left\{Z_{1}, \ldots, Z_{n_{1}}\right\}, \quad V_{2}=\operatorname{span}\left\{Z_{n_{1}+1}, \ldots, Z_{n_{1}+n_{2}}\right\}, \quad \text { and so on. }
$$

We define dilations $\left\{\Delta_{\lambda}\right\}_{\lambda>0}$ on $\operatorname{Lie}(G)$ by considering, if $\lambda>0$, the unique endomorphism $\Delta_{\lambda}$ of $\operatorname{Lie}(G)$ such that (for every $\left.i=1, \ldots, r\right) \Delta_{\lambda}(X)=\lambda^{i} X$ for every $X \in V_{i}$. The number $Q:=\sum_{i=1}^{r} i \operatorname{dim}\left(V_{i}\right)$ is called the homogeneous dimension of $G$ (see also (9.3)). We suppose throughout that $Q \geq 3$. Any operator of the form

$$
X_{1}^{2}+\cdots+X_{n_{1}}^{2} \quad\left(n_{1}=\operatorname{dim}\left(V_{1}\right)\right)
$$

is called a $s u b$-Laplacian on $G$ provided that $\left\{X_{1}, \ldots, X_{n_{1}}\right\}$ is a basis of $V_{1}$. By means of logarithmic (global) coordinates and the identification of $\operatorname{Lie}(G)$ with $\mathbb{R}^{N}$ (via the basis $\mathcal{Z}$ ), we can push forward the Lie group structure of $G$ and the dilations $\Delta_{\lambda}$ to suitable maps on $\mathbb{R}^{N}$ : more precisely, if we set

$$
\varphi: \mathbb{R}^{N} \rightarrow \operatorname{Lie}(G), \quad \varphi\left(x_{1}, \ldots, x_{N}\right):=x_{1} Z_{1}+\cdots+x_{N} Z_{N},
$$

then the map defined by

$$
x * y:=\varphi^{-1}(\log (\operatorname{Exp}(\varphi(x)) \cdot \operatorname{Exp}(\varphi(y)))), \quad x, y \in \mathbb{R}^{N},
$$

equips $\mathbb{R}^{N}$ with the structure of a Lie group $(\mathbb{G}, *)$ which is isomorphic to $(G, \cdot)$ via $\operatorname{Exp} \circ \varphi$. Also, the definition

$$
\delta_{\lambda}: \mathbb{R}^{N} \rightarrow \mathbb{R}^{N}, \quad \delta_{\lambda}(x):=\varphi^{-1}\left(\Delta_{\lambda}(\varphi(x))\right),
$$

turns out to be very useful since one has, unraveling definitions,

$$
\delta_{\lambda}\left(x_{1}, \ldots, x_{N}\right)=\left(\lambda x_{1}, \ldots, \lambda x_{n_{1}}, \lambda^{2} x_{n_{1}+1}, \ldots, \lambda^{2} x_{n_{1}+n_{2}}, \ldots, \lambda^{r} x_{N}\right) .
$$


Note that this gives

$$
\left|\delta_{\lambda}(E)\right|=\lambda^{Q}|E| \quad \text { for every measurable set } E \subseteq \mathbb{R}^{N} .
$$

Since $\Psi:=\varphi^{-1} \circ \log : G \rightarrow \mathbb{G}$ is a group isomorphism, its differential $\mathrm{d} \Psi: \operatorname{Lie}(G) \rightarrow$ $\operatorname{Lie}(\mathbb{G})$ is an algebra isomorphism, so that $\mathfrak{g}:=\operatorname{Lie}(\mathbb{G})$ admits a stratification analogous to that of $G$ : indeed, if we set $W_{i}:=\mathrm{d} \Psi\left(V_{i}\right)$, then $\mathfrak{g}=W_{1} \oplus \cdots \oplus W_{r}$, and

$$
W_{2}=\left[W_{1}, W_{1}\right], \ldots, W_{r}=\left[W_{1}, W_{r-1}\right], \quad \text { and } \quad\left[W_{1}, W_{r}\right]=\{0\} .
$$

As a consequence, if $X_{1}^{2}+\cdots+X_{n_{1}}^{2}$ is a sub-Laplacian on $G$, then

$$
\mathcal{L}:=\left(\mathrm{d} \Psi\left(X_{1}\right)\right)^{2}+\cdots+\left(\mathrm{d} \Psi\left(X_{n_{1}}\right)\right)^{2}
$$

is a sub-Laplacian on $\mathbb{G}$.

The above identifications are not canonical, since they make essential use of the basis $\mathcal{Z}$, but they are very efficient for calculations. Thus, in the following, when we refer to a Carnot group, to its dilations and to a sub-Laplacian, we shall always mean, respectively, $\mathbb{G}=\left(\mathbb{R}^{N}, *\right), \delta_{\lambda}, \mathcal{L}$ as defined in (9.1), (9.2), (9.4). To avoid misunderstanding, we shall call $\mathbb{G}=\left(\mathbb{R}^{N}, *, \delta_{\lambda}\right)$ a homogeneous Carnot group $\left(\right.$ on $\left.\mathbb{R}^{N}\right)$.

To taste the efficiency of working with the above coordinates, we explicitly remark that $*$ has a particularly nice form: indeed, for every $j \in\{1, \ldots, N\}$, the $j$-th coordinate of $x * y$ is given by

$$
(x * y)_{j}=x_{j}+y_{j}+Q_{j}\left(x_{1}, \ldots, x_{j-1}, y_{1}, \ldots, y_{j-1}\right),
$$

for a suitable polynomial function $Q_{j}$ (here $Q_{j}=0$ for $j=1, \ldots, n_{1}$ ). Thus, the Jacobian matrices of the right and left translations on $\mathbb{G} \equiv \mathbb{R}^{N}$ are triangular matrices with 1 's on the main diagonal. This ensures that the Lebesgue measure on $\mathbb{R}^{N}$ is a bi-invariant Haar measure for $\mathbb{G}=\left(\mathbb{R}^{N}, *\right)$. Moreover, any sub-Laplacian on $\mathbb{G}$ can be characterized by the following fact:

$$
\mathcal{L}=X_{1}^{2}+\cdots+X_{m}^{2},
$$

where $\left\{X_{1}, \ldots, X_{m}\right\}$ is a basis for the vector space (called the first layer of $\mathfrak{g}$ ) comprising all the left-invariant vector fields $X$ on $\left(\mathbb{R}^{N}, *\right)$ (thought of, here and henceforth, as linear partial differential operators on $\mathbb{R}^{N}$ ) which are $\delta_{\lambda}$-homogeneous of degree 1 , i.e.,

$$
X(f \circ \varphi)=\lambda(X f) \circ \varphi, \quad \forall f \in C^{\infty}\left(\mathbb{R}^{N}, \mathbb{R}\right), \forall \lambda>0 .
$$

Thus any sub-Laplacian is $\delta_{\lambda}$-homogeneous of degree 2 , with the obvious meaning. Most importantly for our purposes, it is not difficult to prove that (9.5) implies that any subLaplacian is a divergence form operator. Indeed, if we set

$$
X_{j}=\sum_{i=1}^{N} \sigma_{i, j}(x) \frac{\partial}{\partial x_{i}}, \quad j=1, \ldots, m,
$$

and we consider the $N \times m$ matrix $\sigma(x)=\left(\sigma_{i, j}(x)\right)_{i \leq N, j \leq m}$, we have

$$
\mathcal{L}=\operatorname{div}(A(x) \nabla), \quad \text { where } \quad A(x)=\sigma(x) \cdot \sigma^{T}(x) .
$$


It can be easily proved that $a_{1,1}$ is a positive constant, so that axiom (ND) in Section 1 holds. Note also that $A(x)$ is positive semidefinite, and it is positive definite if and only if $m=N$, in which case we recover the Euclidean group $\mathbb{G}=\left(\mathbb{R}^{N},+\right)$ and $\mathcal{L}$ is a (strictly) elliptic operator with constant coefficients (for example, the classical Laplace operator $\left.\Delta=\sum_{j=1}^{N}\left(\partial_{j}\right)^{2}\right)$.

The results in [9, Chapter 5] imply that any sub-Laplacian $\mathcal{L}$ on $\mathbb{G}$ admits a unique fundamental solution $\Gamma$ with the properties listed in Section 1. Furthermore, the results in [9, Chapter 7] prove that the set of $\mathcal{L}$-harmonic functions endows $\mathbb{R}^{N}$ with the structure of a $\mathfrak{S}^{*}$-harmonic space. Hence, all the results of this paper apply to the sub-Laplacians $\mathcal{L}$ on homogeneous Carnot groups.

Mean integral operators for sub-Laplacians. We next study more closely what the mean integral operators of the previous sections look like in the context of sub-Laplacians. As proved in [9, Proposition 5.4.2] (see also Folland [12]), $\Gamma(x, y)$ has the following convenient form:

$$
\Gamma(x, y)=d^{2-Q}\left(x^{-1} * y\right), \quad x, y \in \mathbb{R}^{N}, x \neq y,
$$

where $d$ is a symmetric $\delta_{\lambda}$-homogeneous norm (also referred to as a gauge function), that is: $d \in C\left(\mathbb{R}^{N}, \mathbb{R}\right) \cap C^{\infty}\left(\mathbb{R}^{N} \backslash\{0\}, \mathbb{R}\right) ; d(x) \geq 0$ and $d(x)=0$ iff $x=0 ; d\left(\delta_{\lambda}(x)\right)=$ $\lambda d(x)$ for every $x \in \mathbb{R}^{N}$ and every $\lambda>0 ; d\left(x^{-1}\right)=d(x)$ for every $x \in \mathbb{R}^{N}$, where $x^{-1}$ is the group inversion on $\mathbb{G}$.

Given $x \in \mathbb{R}^{N}$ and $r>0$ we consider the gauge ball of center $x$ and radius $r$, i.e.,

$$
D(x, r):=\left\{y \in \mathbb{R}^{N}: d\left(x^{-1} * y\right)<r\right\} .
$$

Note that, by the left-invariance on $\mathbb{G}$ of the Lebesgue measure and by (9.3), we have

$$
|D(x, r)|=r^{Q}|D(0,1)| \quad \text { for every } x \in \mathbb{G} \text { and every } r>0 .
$$

With reference to our notation $\Omega_{r}(x)$ in (3.1), we have

$$
\Omega_{r}(x)=D\left(x, r^{1 /(Q-2)}\right) .
$$

If $\mathcal{L}=\sum_{j=1}^{m} X_{j}^{2}$, we set $\nabla_{\mathcal{L}} u:=\left(X_{1} u, \ldots, X_{m} u\right)$ for every differentiable function $u$. In view of (9.8), we have

$$
\langle A \nabla u, \nabla u\rangle=\left\langle\sigma^{T} \nabla u, \sigma^{T} \nabla u\right\rangle=\left|\sigma^{T} \nabla u\right|^{2} \stackrel{(9.7)}{=}\left|\left(X_{1} u, \ldots, X_{m} u\right)\right|^{2}=\left|\nabla_{\mathcal{L}} u\right|^{2} .
$$

Note that $\nabla_{\mathcal{L}}$ is a left-invariant operator on $\mathbb{G}$, since all the $X_{j}$ 's belong to the Lie algebra of $\mathbb{G}$. Moreover, since $\nabla_{\mathcal{L}} \Gamma_{x}=\nabla_{\mathcal{L}}\left(d^{2-Q}\right)=(2-Q) d^{1-Q} \nabla_{\mathcal{L}} d$, by simple calculations we can rewrite the kernels $\mathcal{K}$ and $K^{\alpha}$ in (11.6) and (11.14) as follows:

$$
\begin{aligned}
\mathcal{K}(x, y) & =(Q-2) d^{1-Q}\left(x^{-1} * y\right) \frac{\left|\nabla_{\mathcal{L}} d\right|^{2}\left(x^{-1} * y\right)}{\left|\nabla\left(d\left(x^{-1} *\right)\right)(y)\right|}, \\
K^{(\alpha)}(x, y) & =(Q-2)^{2} d\left(x^{-1} * y\right)^{2(1-Q)-(2-Q)(2+\alpha)}\left|\nabla_{\mathcal{L}} d\right|^{2}\left(x^{-1} * y\right),
\end{aligned}
$$


where $x, y \in \mathbb{R}^{N}$ and $x \neq y$. Note that $K^{(\alpha)}(x, y)$ is actually a function of $x^{-1} * y$, whereas this is not true of $\mathcal{K}(x, y)$, a priori. This makes the properties of left-invariance of the surface mean integral operator $m_{r}$ nontrivial.

Here and throughout we make the following choice of $\alpha$ :

$$
\alpha=\frac{2}{Q-2} .
$$

This ensures that the exponent of $d$ in the above expression of $K^{(\alpha)}$ becomes zero, so that (dropping henceforth the notation $\alpha$ )

$$
K(x, y)=(Q-2)^{2}\left|\nabla_{\mathcal{L}} d\right|^{2}\left(x^{-1} * y\right) .
$$

The relevant mean integral operators $m_{r}$ and $M_{r}$ in (11.5) and (11.15) thus become

$$
\begin{aligned}
& m_{r}(u)(x)=\frac{Q-2}{r^{(Q-1) /(Q-2)}} \int_{d\left(x^{-1} * y\right)=r^{1 /(Q-2)}} u(y) \frac{\left|\nabla_{\mathcal{L}} d\right|^{2}\left(x^{-1} * y\right)}{\left|\nabla\left(d\left(x^{-1} * \cdot\right)\right)(y)\right|} \mathrm{d} \sigma(y), \\
& M_{r}(u)(x)=\frac{Q(Q-2)}{r^{Q /(Q-2)}} \int_{d\left(x^{-1} * y\right)<r^{1 /(Q-2)}} u(y)\left|\nabla_{\mathcal{L}} d\right|^{2}\left(x^{-1} * y\right) \mathrm{d} y .
\end{aligned}
$$

In the notation of [9, Section 5.5], if we set

$$
\Psi_{\mathcal{L}}(x):=\left|\nabla_{\mathcal{L}} d\right|^{2}(x), \quad x \neq 0,
$$

and if we scale the radius by letting

$$
\mathcal{M}_{r}(u)(x):=m_{r Q-2}(u)(x) \quad \text { and } \quad \mathrm{M}_{r}(u)(x):=M_{r Q-2}(u)(x),
$$

we obtain

$$
\begin{aligned}
& \mathcal{M}_{r}(u)(x)=\frac{Q-2}{r^{Q-1}} \int_{d\left(x^{-1} * y\right)=r} u(y) \frac{\Psi_{\mathcal{L}}\left(x^{-1} * y\right)}{\left|\nabla\left(d\left(x^{-1} *\right)\right)(y)\right|} \mathrm{d} \sigma(y), \\
& \mathrm{M}_{r}(u)(x)=\frac{Q(Q-2)}{r^{Q}} \int_{d\left(x^{-1} * y\right)<r} u(y) \Psi_{\mathcal{L}}\left(x^{-1} * y\right) \mathrm{d} y .
\end{aligned}
$$

These are precisely the operators introduced in [9, Section 5.5], which played a crucial rôle in our former papers $[5,6,7]$.

Our next task is to prove that-in the context of sub-Laplacians - the "denominators" $q_{r}, Q_{r}, \omega_{r}$ introduced in Theorem 4.2 are all scalar multiples of $r^{2 /(Q-2)}$. This gives a wide generalization of what happens in the Euclidean case (see Remark 3.6), and it also provides a simplification when restating Theorem 4.2 for sub-Laplacians (this restatement is given in Theorem 9.1 below).

To this end, let $x \in \mathbb{R}^{N}$ and $r>0$ be fixed. We have, by (9.12) and (9.3), $\left|\Omega_{r}(x)\right|=$ $\left|D\left(x, r^{1 /(Q-2)}\right)\right|=\left|D\left(0, r^{1 /(Q-2)}\right)\right|=r^{Q /(Q-2)}|D(0,1)|$. This gives

$$
\left|\Omega_{r}(x)\right|=r^{Q /(Q-2)} \varpi_{d}, \quad \text { where } \varpi_{d}:=|\{d(x)<1\}| .
$$


As a consequence, if we consider the integrals representing $q_{r}(x), Q_{r}(x), \omega_{r}(x)$ in formulas (3.7), direct calculations show that

$$
\begin{aligned}
q_{r}(x) & =\frac{(Q-2) \varpi_{d}}{2} r^{2 /(Q-2)}, \\
Q_{r}(x) & =\frac{Q(Q-2) \varpi_{d}}{2(Q+2)} r^{2 /(Q-2)}, \\
\omega_{r}(x) & =\frac{(Q-2) \varpi_{d}}{Q+2} r^{2 /(Q-2)} .
\end{aligned}
$$

As a consequence of all the above calculations, if we restate Theorem 4.2 when $\mathcal{L}$ is a sub-Laplacian, replacing $r$ with $r^{Q-2}$, and taking into account (9.17) and (9.21a)-(9.21c), we obtain Theorem 9.1 below, which improves our former results in [6]. [In the following statement, we set as usual $\Omega(u)=\{x \in \Omega: u(x)>-\infty\}$. Also, $u \in \operatorname{USC}(\Omega)$ is called $\mathcal{M}$-continuous in $\Omega$ if $\lim _{r \rightarrow 0} \mathcal{M}_{r}(u)(x)=u(x)$ for all $x \in \Omega$. Analogously, we define M-continuity. Finally, we let $R(x)=\sup \{r>0: D(x, r) \subseteq \Omega\}$.]

Theorem 9.1. Let $\mathbb{G}=\left(\mathbb{R}^{N}, *, \delta_{\lambda}\right)$ be a homogeneous Carnot group of homogeneous dimension $Q \geq 3$, and let $\mathcal{L}$ be a sub-Laplacian on $\mathbb{G}$. Let $\Gamma$ be the fundamental solution of $\mathcal{L}$ and consider the gauge function d defined by $\Gamma=d^{2-Q}$. Finally, let $\mathcal{M}_{r}, \mathrm{M}_{r}$ be the mean integral operators defined in (9.18) and (9.19). Suppose $\Omega$ is an open subset of $\mathbb{G}$ and let $u: \Omega \rightarrow[-\infty, \infty)$ be an u.s.c. function which is finite at some point of every component of $\Omega$. Then the following conditions are equivalent:

$(\underline{\mathcal{S}})$ Subharmonicity: $u \in \underline{\mathcal{S}}(\Omega)$ with respect to $\mathcal{L}$.

(1) $\mathcal{M}$-submean condition: $u(x) \leq \mathcal{M}_{r}(u)(x)$ for every $x \in \Omega$ and $r \in(0, R(x))$.

(2) M-submean condition: $u(x) \leq \mathrm{M}_{r}(u)(x)$ for every $x \in \Omega$ and $r \in(0, R(x))$.

(3) Blaschke-type condition: $\limsup _{r \rightarrow 0} \frac{\mathcal{M}_{r}(u)(x)-u(x)}{r^{2}} \geq 0$ for every $x \in \Omega(u)$.

(4) Privaloff-type condition: $\limsup _{r \rightarrow 0} \frac{\mathrm{M}_{r}(u)(x)-u(x)}{r^{2}} \geq 0$ for every $x \in \Omega(u)$.

(5) $\mathcal{M}$-monotonicity: $u$ is $\mathcal{M}$-continuous in $\Omega$ and $r \mapsto \mathcal{M}_{r}(u)(x)$ is increasing on $(0, R(x))$ for every $x \in \Omega$.

(6) M-monotonicity: $u$ is M-continuous in $\Omega$ and $r \mapsto \mathrm{M}_{r}(u)(x)$ is increasing on $(0, R(x))$ for every $x \in \Omega$.

(7) Beckenbach-Radó-type condition: $u$ is $\mathcal{M}$-continuous in $\Omega$, and, for any $x \in \Omega$, we have $\mathrm{M}_{r}(u)(x) \leq \mathcal{M}_{r}(u)(x)$ for every $r \in(0, R(x))$.

(8) Reade-type condition: $u$ is $\mathcal{M}$-continuous in $\Omega$, and, for every $x \in \Omega(u)$,

$$
\liminf _{r \rightarrow 0} \frac{\mathcal{M}_{r}(u)(x)-\mathbf{M}_{r}(u)(x)}{r^{2}} \geq 0 .
$$

Next we provide further results on the average operators $\mathcal{M}_{r}, \mathrm{M}_{r}$ in the sub-Laplacian setting. The following result improves Proposition 9.5.4 in [9], where a result of leftcontinuity was obtained, by means of a suitable $\mathcal{L}$-version of the Poisson-Jensen formula. 
Proposition 9.2. Let $\Omega \subseteq \mathbb{R}^{N}$ be open and suppose that $u$ is $\mathcal{L}$-subharmonic in $\Omega$. Then the functions $(0, R(x)) \ni r \mapsto \mathcal{M}_{r}(u)(x), \mathrm{M}_{r}(u)(x)$ are continuous.

Proof. This follows immediately from Proposition 6.10.

Remark 9.3. With the aid of (9.17), (11.13) and (9.14), it is easily seen that the following identity holds for every $u \in \operatorname{USC}(\Omega)$ and every $x, r$ such that $\overline{D(x, r)} \subset \Omega$ :

$$
\mathrm{M}_{r}(u)(x)=\frac{Q}{r^{Q}} \int_{0}^{r} \rho^{Q-1} \mathcal{M}_{\rho}(u)(x) \mathrm{d} \rho .
$$

We next prove the following left-invariance property of the mean integral operators for $\mathcal{L}$. Given $\alpha \in \mathbb{G}$, we set $\tau_{\alpha}: \mathbb{G} \rightarrow \mathbb{G}, \tau_{\alpha}(x):=\alpha * x$.

Proposition 9.4. Let $u \in \operatorname{USC}(\Omega)$ and $\xi \in \mathbb{G}$. Suppose that $\overline{D(\xi * x, R)} \subset \Omega$. Then

$$
\mathrm{M}_{r}\left(u \circ \tau_{\xi}\right)(x)=\mathrm{M}_{r}(u)(\xi * x), \quad \forall r \in(0, R) .
$$

Furthermore, we also have the analogous equality for surface mean integrals,

$$
\mathcal{M}_{r}\left(u \circ \tau_{\xi}\right)(x)=\mathcal{M}_{r}(u)(\xi * x), \quad \forall r \in(0, R),
$$

if both sides are continuous functions of $r \in(0, R)$. This is always the case if $u \in \underline{\mathcal{S}}(\Omega)$.

Proof. Since $u \in \operatorname{USC}(\Omega)$, the hypothesis $\overline{D(\xi * x, r)} \subset \Omega$ ensures that the solid mean $\mathrm{M}_{r}(u)(\xi * x)$ is well-defined. The change of variable $y=\xi * z$ gives

$$
\mathrm{M}_{r}(u)(\xi * x)=\frac{Q(Q-2)}{r^{Q}} \int_{d\left(x^{-1} * z\right)<r} u(\xi * z) \Psi_{\mathcal{L}}\left(x^{-1} * z\right) \mathrm{d} z=\mathrm{M}_{r}\left(u \circ \tau_{\xi}\right)(x) .
$$

This proves (9.23). Taking into consideration (9.22), we can rewrite identity (9.23) as

$$
\int_{0}^{r} \rho^{Q-1} \mathcal{M}_{\rho}\left(u \circ \tau_{\xi}\right)(x) \mathrm{d} \rho=\int_{0}^{r} \rho^{Q-1} \mathcal{M}_{\rho}(u)(\xi * x) \mathrm{d} \rho .
$$

If $\mathcal{M}_{\rho}\left(u \circ \tau_{\xi}\right)(x), \mathcal{M}_{\rho}(u)(\xi * x)$ are continuous in $\rho$, we can differentiate the above identity with respect to $r$, thus getting $r^{Q-1} \mathcal{M}_{r}\left(u \circ \tau_{\xi}\right)(x)=r^{Q-1} \mathcal{M}_{r}(u)(\xi * x)$ for every $r \in(0, R)$. This gives (9.24). Note that, if $u$ is $\mathcal{L}$-subharmonic in $\Omega$, then we infer that $u \circ \tau_{\xi}$ is $\mathcal{L}$-subharmonic on $\xi^{-1} * \Omega$. Hence, Proposition 9.2 ensures that $\mathcal{M}_{\rho}\left(u \circ \tau_{\xi}\right)(x), \mathcal{M}_{\rho}(u)(\xi * x)$ are continuous in $\rho$. This proves the last statement of the proposition.

Remark 9.5. We explicitly write down the result of Lemma 3.4 in the case of subLaplacians, restated for the mean operators on gauge balls. After taking into consideration (9.9), (9.12), (9.14) and (9.17), the representation formula (3.10) turns out to be

$$
\mathcal{M}_{r}(u)(x)-\mathrm{M}_{r}(u)(x)=\frac{Q-2}{2 r Q} \int_{D(x, r)}\left(r^{2}-d^{2}\left(x^{-1} * y\right)\right) \mathcal{L} u(y) \mathrm{d} y
$$

for all $u \in C^{2}(\overline{D(x, r)}, \mathbb{R}), x \in \mathbb{G}$ and $r>0$. 


\section{Saks-type theorems}

The aim of this section is to provide a generalization to the sub-Laplacian setting of a notable result by Saks [31], concerning the value of the limit (4.3) in the Reade-type condition from Theorem 4.2. We begin with a useful lemma, and a proposition containing a weak version of identity (9.25) for $\mathcal{L}$-subharmonic functions.

Lemma 10.1. Let $\Omega \subseteq \mathbb{R}^{N}$ be open and let $u \in \underline{\mathcal{S}}(\Omega)$. If $\overline{D(x, r)} \subset \Omega$, then

$$
\begin{aligned}
& u(x)=\mathcal{M}_{r}(u)(x)-(Q-2) \int_{0}^{r} \frac{\mu_{u}(D(x, t))}{t^{Q-1}} \mathrm{~d} t, \\
& u(x)=\mathrm{M}_{r}(u)(x)-\frac{Q(Q-2)}{r^{Q}} \int_{0}^{r} \rho^{Q-1}\left(\int_{0}^{\rho} \frac{\mu_{u}(D(x, t))}{t^{Q-1}} \mathrm{~d} t\right) \mathrm{d} \rho,
\end{aligned}
$$

where $\mu_{u}$ is the $\mathcal{L}$-Riesz measure of $u$.

Proof. Let $\overline{D(x, r)} \subset \Omega$. First of all, we recall that a representation formula analogous to (3.8) holds when $u \in \mathcal{S}(\Omega)$, by replacing $\mathcal{L} u(y) \mathrm{d} y$ with $\mathrm{d} \mu_{u}(y)$ (see [9, Theorem 9.5.2, p. 447]). That is (see also (9.12) and (9.17)), we have

$$
u(x)=\mathcal{M}_{r}(u)(x)-\int_{D(x, r)}\left(\Gamma(x, y)-r^{2-Q}\right) \mathrm{d} \mu_{u}(y) .
$$

By recalling (9.9), writing $d^{2-Q}\left(x^{-1} * y\right)-r^{2-Q}=-\int_{d\left(x^{-1} * y\right)}^{r}(2-Q) t^{1-Q} \mathrm{~d} t$ and applying Fubini's Theorem, we get

$$
u(x)=\mathcal{M}_{r}(u)(x)+(2-Q) \int_{0}^{r} t^{1-Q}\left(\int_{d\left(x^{-1} * y\right)<t} \mathrm{~d} \mu_{u}(y)\right) \mathrm{d} t,
$$

which is (10.1). Now (10.2) follows from (10.1), in view of (9.22).

The following representation formula (of Poisson-Jensen type) seems to be new, even in the case of the Laplace operator.

Theorem 10.2. Let $\Omega \subseteq \mathbb{R}^{N}$ be open and let $u \in \underline{\mathcal{S}}(\Omega)$. If $\overline{D(x, r)} \subset \Omega$, then

$$
\mathcal{M}_{r}(u)(x)-\mathbf{M}_{r}(u)(x)=\frac{Q-2}{2 r^{Q}} \int_{D(x, r)}\left(r^{2}-d^{2}\left(x^{-1} * y\right)\right) \mathrm{d} \mu_{u}(y),
$$

where $\mu_{u}$ is the $\mathcal{L}$-Riesz measure of $u$. Equivalently,

$$
\mathcal{M}_{r}(u)(x)-\mathrm{M}_{r}(u)(x)=\frac{Q-2}{r Q} \int_{0}^{r} t \mu_{u}(D(x, t)) \mathrm{d} t .
$$

Proof. For any $\varepsilon>0$, we define the open set

$$
\widehat{\Omega}^{\varepsilon}:=\{x \in \Omega: \overline{D(0, \varepsilon)} * x \subset \Omega\} .
$$

Note that $\widehat{\Omega}^{\varepsilon} \uparrow \Omega$ as $\varepsilon \downarrow 0$. Also, for every compact set $K \subset \Omega$, there exists $\varepsilon_{0}>0$ such that $K \subset \widehat{\Omega}^{\varepsilon}$ for every $\varepsilon \in\left(0, \varepsilon_{0}\right]$. We next fix $J \in C_{0}^{\infty}\left(\mathbb{R}^{N}, \mathbb{R}\right)$ with $J \geq 0$, 
$\int J=1$ such that the support of $J$ is a compact subset of $D(0,1)$. Set finally $J_{\varepsilon}(x):=$ $\varepsilon^{-Q} J\left(\delta_{1 / \varepsilon}(x)\right)$ for $\varepsilon>0$. For any $L_{\text {loc }}^{1}$ function $w$ on $\Omega$, we set

$$
w_{\varepsilon}: \Omega^{\varepsilon} \rightarrow \mathbb{R}, \quad w_{\varepsilon}(x):=\int_{d\left(x * y^{-1}\right)<\varepsilon} w(y) J_{\varepsilon}\left(x * y^{-1}\right) \mathrm{d} y .
$$

Note that $\left\{y \in \mathbb{R}^{N}: d\left(x * y^{-1}\right)<\varepsilon\right\}=D(0, \varepsilon) * x$. Hence, for every $x \in \widehat{\Omega}^{\varepsilon}$ and every $y$ such that $d\left(x * y^{-1}\right)<\varepsilon$, we also have $y \in \Omega$, so that (via the change of variable $\left.x * y^{-1}=z\right), w_{\varepsilon}$ can be rewritten as

$$
w_{\varepsilon}(x)=\int_{D(0, \varepsilon)} w\left(z^{-1} * x\right) J_{\varepsilon}(z) \mathrm{d} z, \quad x \in \widehat{\Omega}^{\varepsilon} .
$$

It is easily seen that $w_{\varepsilon} \in C^{\infty}\left(\widehat{\Omega}^{\varepsilon}, \mathbb{R}\right)$ and that $w_{\varepsilon} \rightarrow w$ as $\varepsilon \rightarrow 0$ in $L_{\mathrm{loc}}^{1}(\Omega)$. Moreover, if $w$ is continuous, then $w_{\varepsilon} \rightarrow w$ as $\varepsilon \rightarrow 0$ uniformly on compact subsets of $\Omega$. The following result is a consequence of the left-invariance properties in Proposition 9.4:

Let $u \in \underline{\mathcal{S}}(\Omega)$ and suppose that $\overline{D(x, r)} \subset \Omega$; then

$$
\mathcal{M}_{r}\left(u_{\varepsilon}\right)(x)=\left(\mathcal{M}_{r}(u)\right)_{\varepsilon}(x), \quad \mathbf{M}_{r}\left(u_{\varepsilon}\right)(x)=\left(\mathbf{M}_{r}(u)\right)_{\varepsilon}(x),
$$

for every $\varepsilon>0$ small enough.

Indeed, for every small $\varepsilon>0$ such that $\overline{D(x, r)} \subset \widehat{\Omega}^{\varepsilon}$, we have (temporarily denoting by $\mathcal{K}_{\mathcal{L}}(x, y)$ the kernel $\Psi_{\mathcal{L}}\left(x^{-1} * y\right) /\left|\nabla\left(d\left(x^{-1} * \cdot\right)\right)(y)\right|$ in $\left.(9.18)\right)$ :

$$
\begin{aligned}
\mathcal{M}_{r}\left(u_{\varepsilon}\right)(x) & \stackrel{(10.5)}{=} \frac{Q-2}{r^{Q-1}} \int_{D(x, r)}\left(\int_{D(0, \varepsilon)} u\left(z^{-1} * y\right) J_{\varepsilon}(z) \mathrm{d} z\right) \mathcal{K}_{\mathcal{L}}(x, y) \mathrm{d} \sigma(y) \\
& =\int_{D(0, \varepsilon)} \mathcal{M}_{r}\left(u \circ \tau_{z^{-1}}\right)(x) J_{\varepsilon}(z) \mathrm{d} z
\end{aligned}
$$

(we can use formula (9.23) in Proposition 9.4, as $u \in \underline{\mathcal{S}}(\Omega)$ )

$$
=\int_{D(0, \varepsilon)} \mathcal{M}_{r}(u)\left(z^{-1} * x\right) J_{\varepsilon}(z) \mathrm{d} z \stackrel{(10.5)}{=}\left(\mathcal{M}_{r}(u)\right)_{\varepsilon}(x) .
$$

The case of the solid mean $\mathrm{M}_{r}$ is completely analogous.

We next prove Theorem 10.2. Let $u \in \underline{\mathcal{S}}(\Omega)$ and suppose that $A:=D\left(x_{0}, r\right)$ is such that $\bar{A} \subset \Omega$. There certainly exists $\varepsilon_{0}>0$ such that $\bar{A} \subset \widehat{\Omega}^{\varepsilon_{0}}$. Note that this also ensures that $\bar{A} \subset \widehat{\Omega}^{\varepsilon}$ for every $\varepsilon \in\left(0, \varepsilon_{0}\right)$. For $0<\varepsilon<\varepsilon_{0}$, let us consider the ' $\mathbb{G}$-mollifier' $u_{\varepsilon}$ on $\widehat{\Omega}^{\varepsilon}$. Since $u_{\varepsilon}$ is of class $C^{\infty}$ on $\widehat{\Omega}^{\varepsilon}$, we can apply formula (9.25) to get

$$
\mathcal{M}_{r}\left(u_{\varepsilon}\right)\left(x_{0}\right)-\mathrm{M}_{r}\left(u_{\varepsilon}\right)\left(x_{0}\right)=\frac{Q-2}{2 r^{Q}} \int_{D\left(x_{0}, r\right)}\left(r^{2}-d^{2}\left(x_{0}^{-1} * y\right)\right) \mathcal{L}\left(u_{\varepsilon}\right)(y) \mathrm{d} y .
$$

We claim that, letting $\varepsilon \rightarrow 0$ in (10.7), we obtain (10.3).

The proof is quite laborious. First of all, we remark that

$$
\lim _{\varepsilon \rightarrow 0} \mathcal{M}_{r}\left(u_{\varepsilon}\right)\left(x_{0}\right)=\mathcal{M}_{r}(u)\left(x_{0}\right), \quad \lim _{\varepsilon \rightarrow 0} \mathrm{M}_{r}\left(u_{\varepsilon}\right)\left(x_{0}\right)=\mathrm{M}_{r}(u)\left(x_{0}\right) .
$$


This easily follows from (10.6), the continuity of $\mathcal{M}_{r}(u), \mathbf{M}_{r}(u)$ (see Theorem 6.12) and the fact that (if $w$ is continuous) $\lim _{\varepsilon \rightarrow 0} w_{\varepsilon}=w$ locally uniformly.

We next turn to the right-hand member of (10.7). Set $f(y):=\frac{Q-2}{2 r Q}\left(r^{2}-d^{2}\left(x_{0}^{-1} * y\right)\right)$. We are left to prove that

$$
\lim _{\varepsilon \rightarrow 0} \int_{A} f(y) \mathcal{L}\left(u_{\varepsilon}\right)(y) \mathrm{d} y=\int_{A} f(y) \mathrm{d} \mu_{u}(y) .
$$

Note that $f \in C(\bar{A}, \mathbb{R})$ and $f$ is null on $\partial A$. So, we can prolong $f$ to be identically 0 outside $A$, and we get a continuous function (which we still denote by $f$ ) supported in $\bar{A}$. Hence, there exists a sequence $\psi_{j} \in C_{0}^{\infty}\left(\mathbb{R}^{N}, \mathbb{R}\right)$, compactly supported in $A$, such that

$$
\lim _{j \rightarrow \infty} \sup _{\mathbb{R}^{N}}\left|\psi_{j}-f\right|=0
$$

Let $\delta>0$ be arbitrary. By the uniform continuity of $f$ on $\mathbb{R}^{N}$ (and by simple topological considerations on the structure of $\mathbb{G}$ and on the gauge balls), there exists $\bar{\varepsilon} \in\left(0, \varepsilon_{0}\right)$ (with $\bar{\varepsilon}$ only depending on $\delta$ and $f$ ) such that

$$
|f(x)-f(\eta)|<\delta \quad \text { for every } x, \eta \text { such that } x \in D(0, \bar{\varepsilon}) * \eta
$$

By (10.9), there exists $j(\delta) \in \mathbb{N}$ such that

$$
\left|\psi_{j}(x)-f(x)\right|<\delta \quad \text { for every } x \in \mathbb{R}^{N} \text { and every } j \geq j(\delta) \text {. }
$$

Gathering together (10.10) and (10.11), for the same $\bar{\varepsilon}, j(\delta)$ as above, we have

$$
\begin{gathered}
\left|\psi_{j}(x)-\psi_{j}(\eta)\right|<3 \delta \quad \text { for every } x, \eta \text { with } x \in D(0, \bar{\varepsilon}) * \eta \\
\text { and every } j \geq j(\delta) .
\end{gathered}
$$

Let $\varepsilon \in(0, \bar{\varepsilon})$ be given. By (10.9), we have $\lim _{j \rightarrow \infty} \int_{A} \psi_{j} \mathcal{L}\left(u_{\varepsilon}\right)=\int_{A} f \mathcal{L}\left(u_{\varepsilon}\right)$, so that there certainly exists an integer $j(\delta, \varepsilon) \geq j(\delta)$ such that

$$
\left|\int_{A} \psi_{j} \mathcal{L}\left(u_{\varepsilon}\right)-\int_{A} f \mathcal{L}\left(u_{\varepsilon}\right)\right|<\delta \quad \text { for every } j \geq j(\delta, \varepsilon) .
$$

On the other hand, $\psi_{j}$ being compactly supported in $A$ and $u_{\varepsilon}$ being smooth on $A$, we can integrate by parts to get

$$
\int_{A} \psi_{j} \mathcal{L}\left(u_{\varepsilon}\right)=\int_{A} u_{\varepsilon} \mathcal{L}\left(\psi_{j}\right) \text { for every } j \text { and } \varepsilon
$$


Furthermore we have the following representation of the right-hand side of (10.14):

$$
\begin{aligned}
\int_{A} u_{\varepsilon} \mathcal{L} \psi_{j} & \stackrel{(10.5)}{=} \int_{D(0, \varepsilon)}\left(\int_{A} \mathcal{L} \psi_{j}(y) u\left(z^{-1} * y\right) \mathrm{d} y\right) J_{\varepsilon}(z) \mathrm{d} z \\
& \text { (we use the change of variable } \left.\eta=z^{-1} * y \text { and the left-invariance of } \mathcal{L}\right) \\
& =\int_{D(0, \varepsilon)}\left(\int_{z^{-1} * A} \mathcal{L}\left(\psi_{j} \circ \tau_{z}\right)(\eta) u(\eta) \mathrm{d} \eta\right) J_{\varepsilon}(z) \mathrm{d} z \\
& \text { (recall that } \left.\bar{A} \subset \widehat{\Omega}^{\varepsilon} \text { so that } z^{-1} * A \subset \Omega \text { for every } z \in D(0, \varepsilon)\right) \\
& =\int_{D(0, \varepsilon)}\left(\int_{\Omega} \mathcal{L}\left(\psi_{j} \circ \tau_{z}\right)(\eta) u(\eta) \mathrm{d} \eta\right) J_{\varepsilon}(z) \mathrm{d} z \\
& \stackrel{(6.1)}{=} \int_{D(0, \varepsilon)}\left(\int_{\Omega} \psi_{j}(z * \eta) \mathrm{d} \mu_{u}(\eta)\right) J_{\varepsilon}(z) \mathrm{d} z \\
& =\int_{\Omega}\left(\int_{D(0, \varepsilon) * \eta} \psi_{j}(x) J_{\varepsilon}\left(x * \eta^{-1}\right) \mathrm{d} x\right) \mathrm{d} \mu_{u}(\eta) .
\end{aligned}
$$

By exploiting the fact that $\psi_{j}=0$ outside $A$, this gives

$$
\int_{A} u_{\varepsilon} \mathcal{L} \psi_{j}=\int_{D(0, \varepsilon) * A}\left(\int_{D(0, \varepsilon) * \eta} \psi_{j}(x) J_{\varepsilon}\left(x * \eta^{-1}\right) \mathrm{d} x\right) \mathrm{d} \mu_{u}(\eta) .
$$

Let finally $\varepsilon \in(0, \bar{\varepsilon})$ be arbitrary and let $j(\delta, \varepsilon) \in \mathbb{N}$ be as in (10.13). We have

$$
\begin{aligned}
& \left|\int_{A} f \mathcal{L}\left(u_{\varepsilon}\right)-\int_{A} f \mathrm{~d} \mu_{u}\right| \\
& \leq\left|\int_{A} f \mathcal{L}\left(u_{\varepsilon}\right)-\int_{A} \psi_{j(\delta, \varepsilon)} \mathcal{L}\left(u_{\varepsilon}\right)\right|+\left|\int_{A} \psi_{j(\delta, \varepsilon)} \mathcal{L}\left(u_{\varepsilon}\right)-\int_{A} f \mathrm{~d} \mu_{u}\right| \\
& \stackrel{(10.13)}{\leq} \delta+\left|\int_{A} \psi_{j(\delta, \varepsilon)} \mathcal{L}\left(u_{\varepsilon}\right)-\int_{A} f \mathrm{~d} \mu_{u}\right| \stackrel{(10.14)}{=} \delta+\left|\int_{A} u_{\varepsilon} \mathcal{L}\left(\psi_{j(\delta, \varepsilon)}\right)-\int_{A} f \mathrm{~d} \mu_{u}\right| \\
& \leq \delta+\left|\int_{A} u_{\varepsilon} \mathcal{L}\left(\psi_{j(\delta, \varepsilon)}\right)-\int_{A} \psi_{j(\delta, \varepsilon)} \mathrm{d} \mu_{u}\right|+\left|\int_{A} \psi_{j(\delta, \varepsilon)} \mathrm{d} \mu_{u}-\int_{A} f \mathrm{~d} \mu_{u}\right| \\
& \stackrel{(10.11)}{\leq} \delta+\delta \mu_{u}(A)+\left|\int_{A} u_{\varepsilon} \mathcal{L}\left(\psi_{j(\delta, \varepsilon)}\right)-\int_{A} \psi_{j(\delta, \varepsilon)} \mathrm{d} \mu_{u}\right| \\
& \stackrel{(10.15)}{=} \delta+\delta \mu_{u}(A) \\
& +\left|\int_{D(0, \varepsilon) * A}\left(\int_{D(0, \varepsilon) * \eta} \psi_{j(\delta, \varepsilon)}(x) J_{\varepsilon}\left(x * \eta^{-1}\right) \mathrm{d} x\right) \mathrm{d} \mu_{u}(\eta)-\int_{A} \psi_{j(\delta, \varepsilon)} \mathrm{d} \mu_{u}\right| .
\end{aligned}
$$

We can proceed with the foregoing chain of inequalities by noticing that the third summand on the right-hand side equals

$$
\left|\int_{D(0, \varepsilon) * A}\left(\int_{D(0, \varepsilon) * \eta}\left(\psi_{j(\delta, \varepsilon)}(x)-\psi_{j(\delta, \varepsilon)}(\eta)\right) J_{\varepsilon}\left(x * \eta^{-1}\right) \mathrm{d} x\right) \mathrm{d} \mu_{u}(\eta)\right|,
$$


because $\int_{D(0, \varepsilon) * \eta} J_{\varepsilon}\left(x * \eta^{-1}\right) \mathrm{d} x=1$, and $\int_{A} \psi_{j(\delta, \varepsilon)} \mathrm{d} \mu_{u}=\int_{D(0, \varepsilon) * A} \psi_{j(\delta, \varepsilon)} \mathrm{d} \mu_{u}$ (as $\psi_{j(\delta, \varepsilon)} \equiv 0$ outside $A$ ). In turn, since $J_{\varepsilon} \geq 0$, by using (10.12) (recall that $\varepsilon \leq \bar{\varepsilon}$ and $j(\delta, \varepsilon) \geq j(\delta))$ we can majorize the above quantity by

$$
\begin{aligned}
\int_{D(0, \varepsilon) * A}\left(\int_{D(0, \varepsilon) * \eta} 3 \delta J_{\varepsilon}\left(x * \eta^{-1}\right) \mathrm{d} x\right) \mathrm{d} \mu_{u}(\eta) & =3 \delta \int_{D(0, \varepsilon) * A} \mathrm{~d} \mu_{u}(\eta) \\
& =3 \delta \mu_{u}(D(0, \varepsilon) * A) \leq 3 \delta \mu_{u}\left(D\left(0, \varepsilon_{0}\right) * A\right) .
\end{aligned}
$$

Gathering together all the above estimates, we have proved that, for every $\delta>0$ there exists $\bar{\varepsilon}$ only depending on $\delta$ such that, for every $\varepsilon \in(0, \bar{\varepsilon})$,

$$
\left|\int_{A} f \mathcal{L}\left(u_{\varepsilon}\right)-\int_{A} f \mathrm{~d} \mu_{u}\right| \leq \delta+\delta \mu_{u}(A)+3 \delta \mu_{u}\left(D\left(0, \varepsilon_{0}\right) * A\right) .
$$

This is exactly the claimed (10.8), and the proof of identity (10.3) is complete.

Finally, the right-hand side of (10.4) coincides with that of (10.3): indeed,

$$
\begin{aligned}
& \frac{Q-2}{2 r^{Q}} \int_{D(x, r)}\left(r^{2}-d^{2}\left(x^{-1} * y\right)\right) \mathrm{d} \mu_{u}(y) \\
& \quad=\frac{Q-2}{r^{Q}} \int_{0}^{r} t\left(\int_{d\left(x^{-1} * y\right)<t} \mathrm{~d} \mu_{u}(y)\right) \mathrm{d} t=\frac{Q-2}{r^{Q}} \int_{0}^{r} t \mu_{u}(D(x, t)) \mathrm{d} t .
\end{aligned}
$$

This completes the proof.

We are ready to prove the following theorem, which generalizes (to the case of subLaplacians) and improves a result due to Saks [31, Theorem 4, p. 454]. Our proof, even in the classical case, is simpler than Saks's.

In the following statement, given a Radon measure $\mu$ on an open set $\Omega$ and given $x \in \Omega$, we define the $d$-symmetric derivative of $\mu$ at $x$ to be the following limit, when it exists:

$$
D_{\mathrm{s}} \mu(x):=\lim _{r \rightarrow 0} \frac{\mu(D(x, r))}{H_{N}(D(x, r))} .
$$

Here, as usual, $H_{N}$ denotes the Lebesgue measure on $\mathbb{R}^{N}$.

Theorem 10.3 (Saks-type theorem for sub-Laplacians). Let $\Omega \subseteq \mathbb{R}^{N}$ be open and let $u \in \underline{\mathcal{S}}(\Omega)$. Let $\mathrm{d} \mu_{u}(y)=\varphi(y) \mathrm{d} y+\mathrm{d} s(y)$ be the Lebesgue decomposition of the $\mathcal{L}$-Riesz measure of $u$ (where $\varphi \in L_{\mathrm{loc}}^{1}(\Omega), \varphi \geq 0$, and $s$ is an $H_{N}$-singular Radon measure on $\Omega$ ). Finally, let $\varpi_{d}$ be the constant in (9.20). Suppose that the $d$-symmetric derivative $D_{\mathrm{s}} \mu_{u}(x)$ exists. Then the following limits exist and we have the equality

$$
\lim _{r \rightarrow 0} \frac{\mathcal{M}_{r}(u)(x)-u(x)}{\frac{(Q-2) \varpi_{d}}{2} r^{2}}=\lim _{r \rightarrow 0} \frac{\mathrm{M}_{r}(u)(x)-u(x)}{\frac{Q(Q-2) \varpi_{d}}{2(Q+2)} r^{2}}=D_{\mathrm{s}} \mu_{u}(x) .
$$

This holds true for $H_{N}$-almost every $x \in \Omega$, since $D_{\mathrm{s}} \mu_{u}(x)=\varphi(x) H_{N}$-a.e. 
Proof. In the notation of the above statement, by exploiting (10.1) and (9.11) we infer

$$
\frac{\mathcal{M}_{r}(u)(x)-u(x)}{r^{2}}=\frac{Q-2}{r^{2}} \int_{0}^{r} \frac{\mu_{u}(D(x, t))}{t^{Q-1}} \mathrm{~d} t=\frac{(Q-2) \varpi_{d}}{r^{2}} \int_{0}^{r} t \frac{\mu_{u}(D(x, t))}{H_{N}(D(x, t))} \mathrm{d} t .
$$

Hence, if $D_{\mathrm{s}} \mu_{u}(x)$ exists, we obviously have

$$
\lim _{r \rightarrow 0} \frac{\mathcal{M}_{r}(u)(x)-u(x)}{r^{2}}=\frac{(Q-2) \varpi_{d}}{2} D_{\mathrm{s}} \mu_{u}(x) .
$$

With the aid of (10.2), and by taking into account that

$$
\frac{1}{r^{2+Q}} \int_{0}^{r} \rho^{Q-1}\left(\int_{0}^{\rho} t \mathrm{~d} t\right) \mathrm{d} \rho=\frac{1}{2(Q+2)},
$$

we can analogously prove that

$$
\lim _{r \rightarrow 0} \frac{\mathrm{M}_{r}(u)(x)-u(x)}{r^{2}}=\frac{Q(Q-2) \varpi_{d}}{2(Q+2)} D_{\mathrm{s}} \mu_{u}(x) .
$$

This proves the first part of the theorem.

By the Lebesgue decomposition theorem, there exist a nonnegative function $\varphi \in$ $L_{\text {loc }}^{1}(\Omega)$ and an $H_{N}$-singular Radon measure $s$ on $\Omega$ such that $\mathrm{d} \mu_{u}(y)=\varphi(y) \mathrm{d} y+\mathrm{d} s(y)$ on $\Omega$. Moreover, for $H_{N}$-almost every $x \in \Omega$, we have

$$
\frac{1}{|D(x, r)|} \int_{D(x, r)} \varphi(y) \mathrm{d} y \stackrel{r \rightarrow 0}{\longrightarrow} \varphi(x), \quad \frac{s(D(x, r))}{|D(x, r)|} \stackrel{r \rightarrow 0}{\longrightarrow} 0 .
$$

The proof of (10.17) is omitted. [It follows, e.g., by adapting the classical arguments in [30, Chapter 8], replacing the Euclidean metric by the quasi-distance $d$. We explicitly remark that the doubling property of the gauge balls ensures a suitable $d$-version of the Vitali covering lemma (see, e.g., [17, 32]), which is the crucial point in adapting the cited classical results.]

For any $x$ for which (10.17) holds, we thus have $D_{\mathrm{s}} \mu_{u}(x)=\varphi(x)$, whence $D_{\mathrm{s}} \mu_{u}(x)$ $=\varphi(x)$ for $H_{N}$-almost every $x \in \Omega$. This completes the proof.

The following further Saks-type theorem is a consequence of Theorem 10.4 (or, directly, of our Poisson-Jensen type formula (10.4)).

Corollary 10.4. In the hypotheses and notation of Theorem 10.3, we have

$$
\lim _{r \rightarrow 0} \frac{\mathcal{M}_{r}(u)(x)-\mathbf{M}_{r}(u)(x)}{\frac{(Q-2) \varpi_{d}}{Q+2} r^{2}}=D_{\mathrm{s}} \mu_{u}(x)=\varphi(x)
$$

for $H_{N}$-almost every $x \in \Omega$.

Proof. This follows by writing $\left(\mathcal{M}_{r}(u)(x)-\mathrm{M}_{r}(u)(x)\right) / r^{2}=\left(\mathcal{M}_{r}(u)(x)-u(x)\right) / r^{2}-$ $\left(\mathrm{M}_{r}(u)(x)-u(x)\right) / r^{2}$, and by using (10.16). Alternatively, we can argue directly as in Theorem 10.3, this time exploiting formula (10.4). 


\section{Appendix. Proof of the $\mathcal{L}$-representation formulas}

The aim of this appendix is to prove Theorem 3.3, containing the mean value formulas for $\mathcal{L}$ used in this paper.

To begin with, let $\Omega \subset \mathbb{R}^{N}$ be a bounded open set with $\partial \Omega$ of class $C^{1}$. If $u, d \in$ $C^{2}(\bar{\Omega}, \mathbb{R})$, we can apply the Divergence Theorem and derive from (1.1) the following Green-type identity (recall that $A$ is symmetric):

$$
\int_{\Omega}(u \mathcal{L} d-d \mathcal{L} u) \mathrm{d} y=\int_{\partial \Omega}(u\langle A \nabla d, v\rangle-d\langle A \nabla u, v\rangle) \mathrm{d} \sigma .
$$

(Here $v$ denotes the outer normal unit vector on $\partial \Omega$.) The choice $d \equiv 1$ yields

$$
\int_{\Omega} \mathcal{L} u \mathrm{~d} y=\int_{\partial \Omega}\langle A \nabla u, v\rangle \mathrm{d} \sigma, \quad \forall u \in C^{2}(\bar{\Omega}, \mathbb{R}) .
$$

This proves in particular that

$$
\int_{\Omega} \mathcal{L} u \mathrm{~d} y=0 \quad \text { for every } u \in C^{2}(\bar{\Omega}, \mathbb{R}) \text { supported in } \Omega .
$$

Let $x \in \mathbb{R}^{N}$ and $r>0$ be fixed and consider the (regular) open set

$$
\Omega_{r, \rho}:=\Omega_{r}(x) \backslash \overline{\Omega_{\rho}(x)} \text { for } 0<\rho<r .
$$

Let $u \in C^{2}\left(\overline{\Omega_{r}(x)}, \mathbb{R}\right)$ and choose $d(y)=\Gamma_{x}(y):=\Gamma(x, y)$. We can apply (11.1) when $\Omega=\Omega_{r, \rho}$. Recalling that $\mathcal{L} \Gamma_{x}=0$ in $\mathbb{R}^{N} \backslash\{x\}$, and noticing that $\nu=\mp \nabla \Gamma_{x} /\left|\nabla \Gamma_{x}\right|$ on $\partial \Omega_{r}(x)$ and on $\partial \Omega_{\rho}(x)$, respectively, we obtain

$$
\begin{aligned}
-\int_{\Omega_{r, \rho}} \Gamma_{x} \mathcal{L} u= & -\int_{\partial \Omega_{r}(x)} u \frac{\left\langle A \nabla \Gamma_{x}, \nabla \Gamma_{x}\right\rangle}{\left|\nabla \Gamma_{x}\right|} \mathrm{d} \sigma-\int_{\partial \Omega_{r}(x)} \frac{1}{r}\langle A \nabla u, v\rangle \mathrm{d} \sigma \\
& +\int_{\partial \Omega_{\rho}(x)} u \frac{\left\langle A \nabla \Gamma_{x}, \nabla \Gamma_{x}\right\rangle}{\left|\nabla \Gamma_{x}\right|} \mathrm{d} \sigma+\frac{1}{\rho} \int_{\partial \Omega_{\rho}(x)}\langle A \nabla u, v\rangle \mathrm{d} \sigma \\
= & -m_{r}(u)(x)-\mathrm{J}_{r}(u)(x)+m_{\rho}(u)(x)+\mathrm{J}_{\rho}(u)(x) .
\end{aligned}
$$

Here we have set $\mathrm{J}_{r}(u)(x):=\frac{1}{r} \int_{\partial \Omega_{r}(x)}\langle A \nabla u, v\rangle \mathrm{d} \sigma$ and

$$
m_{r}(u)(x):=\int_{\partial \Omega_{r}(x)} u(y) \mathcal{K}(x, y) \mathrm{d} \sigma(y),
$$

where for convenience we denoted by $\mathcal{K}(x, y)$ the following nonnegative kernel:

$$
\mathcal{K}(x, y):=\frac{\left\langle A(y) \nabla \Gamma_{x}(y), \nabla \Gamma_{x}(y)\right\rangle}{\left|\nabla \Gamma_{x}(y)\right|}, \quad x, y \in \mathbb{R}^{N}, x \neq y .
$$

Note that $\mathcal{K}(x, y) \geq 0$ for every $x \neq y$ since we assumed that $A$ is positive semidefinite. 
We now aim to let $\rho$ tend to 0 in (11.4). As for the left-hand side of (11.4), we obviously have (recall that $\Gamma$ is in $L_{\text {loc }}^{1}$ )

$$
-\int_{\Omega_{r}(x) \backslash \overline{\Omega_{\rho}(x)}} \Gamma_{x} \mathcal{L} u \stackrel{\rho \rightarrow 0}{\longrightarrow}-\int_{\Omega_{r}(x)} \Gamma(x, y) \mathcal{L} u(y) \mathrm{d} y .
$$

Moreover, thanks to (11.2) we have $\mathrm{J}_{r}=\frac{1}{r} \int_{\Omega_{r}(x)} \mathcal{L} u$, so that, in view of (3.3),

$$
\lim _{\rho \rightarrow 0} \mathbf{J}_{\rho}=\lim _{\rho \rightarrow 0} \frac{\int_{\Omega_{\rho}(x)} \mathcal{L} u}{\rho}=0, \quad \text { since } \mathcal{L} u \in L^{\infty}\left(\overline{\Omega_{r}(x)}\right) .
$$

Thus $\lim _{\rho \rightarrow 0} m_{\rho}(u)(x)$ exists and (11.4) can be rewritten as

$$
-\int_{\Omega_{r}(x)} \Gamma(x, \cdot) \mathcal{L} u=-m_{r}(u)(x)-\frac{1}{r} \int_{\Omega_{r}(x)} \mathcal{L} u+\lim _{\rho \rightarrow 0} m_{\rho}(u)(x) .
$$

Before we can calculate the limit of $m_{\rho}(u)(x)$, we need some preliminary work.

Suppose that $u \in C_{0}^{\infty}\left(\mathbb{R}^{N}, \mathbb{R}\right)$ and choose $r>0$ large enough so that the support of $u$ is contained in $\Omega_{r}(x)$ (see (3.2)). Note that the left-hand side of (11.7) equals $-\int_{\mathbb{R}^{N}} \Gamma(x, y) \mathcal{L} u(y)=u(x)$, since $\Gamma$ is a fundamental solution for $\mathcal{L}$ (see $\left.(2.1)\right)$. Moreover, the first summand of the right-hand member of (11.7) is null, since $u=0$ on $\partial \Omega_{r}(x)$. The same is true of the second summand, thanks to (11.3). As a consequence, with the assumption that $u$ is smooth and supported in $\Omega_{r}(x),(11.7)$ is equivalent to

$$
u(x)=\lim _{\rho \rightarrow 0} m_{\rho}(u)(x) .
$$

A simple argument of cut-off functions implies that (11.8) also holds for every $u \in$ $C^{\infty}\left(\mathbb{R}^{N}, \mathbb{R}\right)$ and any $x \in \mathbb{R}^{N}$, when $u$ is not necessarily compactly supported. In particular, choosing $u \equiv 1$ gives

$$
\lim _{\rho \rightarrow 0} \int_{\partial \Omega_{\rho}(x)} \mathcal{K}(x, y) \mathrm{d} \sigma(y)=1 \quad \text { for every } x \in \mathbb{R}^{N} .
$$

This allows us to remove the hypothesis of smoothness of $u$ in (11.8) and replace it with the sole continuity of $u$. Indeed, if $u \in C\left(\Omega_{r}(x), \mathbb{R}\right)$, we have

$$
m_{\rho}(u)(x)=\int_{\partial \Omega_{\rho}(x)}(u(y)-u(x)) \mathcal{K}(x, y) \mathrm{d} \sigma(y)+u(x) \int_{\partial \Omega_{\rho}(x)} \mathcal{K}(x, y) \mathrm{d} \sigma(y),
$$

and, as $\rho \rightarrow 0$, the second summand tends to $u(x)$ (thanks to (11.9)), whereas the first summand vanishes. This last fact comes from the following argument: If $u$ is continuous, given $\varepsilon>0$, the first identity in (3.2) ensures that we can find $\bar{\rho}>0$ so small that $\sup _{y \in \partial \Omega_{\rho}(x)}|u(y)-u(x)|<\varepsilon$ for every $\rho \in(0, \bar{\rho})$; hence

$$
\int_{\partial \Omega_{\rho}(x)}|(u(y)-u(x)) \mathcal{K}(x, y)| \mathrm{d} \sigma(y) \leq \varepsilon \int_{\partial \Omega_{\rho}(x)} \mathcal{K}(x, y) \mathrm{d} \sigma(y) \stackrel{\rho \rightarrow 0}{\longrightarrow} \varepsilon .
$$


Here we again applied (11.9) jointly with the nonnegativity of $\mathcal{K}$. We thus have

$$
\lim _{\rho \rightarrow 0} m_{\rho}(u)(x)=u(x) \quad \text { for every } u \in C\left(\Omega_{r}(x), \mathbb{R}\right) .
$$

Let us now go back to the case $u \in C^{2}\left(\overline{\Omega_{r}(x)}, \mathbb{R}\right)$. Inserting (11.10) in (11.7) gives

$$
u(x)=m_{r}(u)(x)-\int_{\Omega_{r}(x)}\left(\Gamma(x, y)-\frac{1}{r}\right) \mathcal{L} u(y) \mathrm{d} y .
$$

This is the surface mean value formula for $\mathcal{L}$ in (3.8).

Note that (11.11) improves (11.9): indeed, taking $u \equiv 1$ in (3.8), yields

$$
m_{r}(1)(x)=\int_{\partial \Omega_{r}(x)} \mathcal{K}(x, y) \mathrm{d} \sigma(y)=1 \quad \text { for every } x \in \mathbb{R}^{N} \text { and } r>0 .
$$

We next introduce solid mean integral operators, by a superposition argument. Throughout, $\alpha>0$ will denote a fixed constant, frequently omitted in the notation. We set

$$
M_{r}(u)(x):=\frac{\alpha+1}{r^{\alpha+1}} \int_{0}^{r} \rho^{\alpha} m_{\rho}(u)(x) \mathrm{d} \rho .
$$

When we need to highlight the rôle of $\alpha$, we shall also write $M_{r}^{(\alpha)}(u)(x):=M_{r}(u)(x)$. Rewriting $\partial \Omega_{r}(x)=\{y: 1 / \Gamma(x, y)=r\}$, inserting the actual expression (11.6) of $\mathcal{K}$ in the definition (11.5) of $m_{\rho}(u)(x)$, and applying Federer's coarea formula, we obtain for $M_{r}(u)(x)$ the following formula:

$$
\begin{gathered}
\frac{\alpha+1}{r^{\alpha+1}} \int_{0}^{r} \rho^{\alpha}\left(\int_{1 / \Gamma(x, y)=\rho} u(y)\left\langle A(y) \nabla \Gamma_{x}(y), \nabla \Gamma_{x}(y)\right\rangle \frac{\mathrm{d} \sigma(y)}{\left|\nabla \Gamma_{x}(y)\right|}\right) \mathrm{d} \rho \\
\left(\operatorname{set} f(y)=1 / \Gamma(x, y) \text { and note that } \nabla \Gamma_{x}(y)=-\Gamma^{2}(x, y)(\nabla f)(y)\right) \\
=\frac{\alpha+1}{r^{\alpha+1}} \int_{0}^{r}\left(\int_{f(y)=\rho} u(y) \frac{\left\langle A(y) \nabla \Gamma_{x}(y), \nabla \Gamma_{x}(y)\right\rangle}{\Gamma(x, y)^{2+\alpha}} \frac{\mathrm{d} \sigma(y)}{|\nabla f(y)|}\right) \mathrm{d} \rho \\
=\frac{\alpha+1}{r^{\alpha+1}} \int_{0<f(y)<r} u(y) \frac{\left\langle A(y) \nabla \Gamma_{x}(y), \nabla \Gamma_{x}(y)\right\rangle}{\Gamma(x, y)^{2+\alpha}} \mathrm{d} y .
\end{gathered}
$$

Recalling that $f=(\Gamma(x, y))^{-1}$ and setting for brevity

$$
K^{(\alpha)}(x, y):=\frac{\left\langle A(y) \nabla \Gamma_{x}(y), \nabla \Gamma_{x}(y)\right\rangle}{\Gamma^{2+\alpha}(x, y)}
$$

(the notation $K(x, y)$ will also be applied), we have proved that

$$
M_{r}^{(\alpha)}(u)(x)=\frac{\alpha+1}{r^{\alpha+1}} \int_{\Omega_{r}(x)} u(y) K^{(\alpha)}(x, y) \mathrm{d} y .
$$

By (11.12) (and the very definition (11.13) of $M_{r}$ ), we obtain the analogous identity

$$
M_{r}(1)(x)=\frac{\alpha+1}{r^{\alpha+1}} \int_{\Omega_{r}(x)} K(x, y) \mathrm{d} y=1, \quad \forall x \in \mathbb{R}^{N}, r>0 .
$$


Also, this time appealing to (11.10), we have

$$
\lim _{\rho \rightarrow 0} M_{\rho}(u)(x)=u(x) \quad \text { for every } u \in C\left(\Omega_{r}(x), \mathbb{R}\right) .
$$

Finally, replacing in (11.11) $r$ with $\rho$, multiplying both sides of $(11.11)$ by $\frac{\alpha+1}{r^{\alpha+1}} \rho^{\alpha}$, and integrating with respect to $\rho \in[0, r]$, we straightforwardly obtain

$$
u(x)=M_{r}^{(\alpha)}(u)(x)-\frac{\alpha+1}{r^{\alpha+1}} \int_{0}^{r} \rho^{\alpha}\left(\int_{\Omega_{\rho}(x)}\left(\Gamma(x, y)-\frac{1}{\rho}\right) \mathcal{L} u(y) \mathrm{d} y\right) \mathrm{d} \rho,
$$

valid for every $u \in C\left(\overline{\Omega_{r}(x)}, \mathbb{R}\right)$, which is our solid mean value formula for $\mathcal{L}$ in (3.9).

With reference to the second summand in the right-hand member of the surface mean value formula (11.11), we set

$$
q_{r}(x):=\int_{\Omega_{r}(x)}\left(\Gamma(x, y)-\frac{1}{r}\right) \mathrm{d} y, \quad x \in \mathbb{R}^{N}, r>0 .
$$

Also, this time referring to the second summand on the right-hand side of our solid mean value formula (11.18), we

$$
Q_{r}(x):=\frac{\alpha+1}{r^{\alpha+1}} \int_{0}^{r} \rho^{\alpha}\left(\int_{\Omega_{\rho}(x)}\left(\Gamma(x, y)-\frac{1}{\rho}\right) \mathrm{d} y\right) \mathrm{d} \rho,
$$

where, as usual, $\alpha>0$ is fixed, whereas $x \in \mathbb{R}^{N}$ and $r>0$ are arbitrary. From the very definitions of $Q_{r}$ and $q_{r}$, we have

$$
Q_{r}(x)=\frac{\alpha+1}{r^{\alpha+1}} \int_{0}^{r} \rho^{\alpha} q_{\rho}(x) \mathrm{d} \rho .
$$

Note that, by definition of $\Omega_{r}(x)=\{\Gamma(x, y)>1 / r\}$ (and the continuity of $\Gamma$ ), we infer that $q_{r}(x)$ and $Q_{r}(x)$ are strictly positive, for any $x \in \mathbb{R}^{N}$ and $r>0$. Also, they are finite since $\Gamma(x, \cdot)$ is locally summable.

Remark 11.1. In the notation of (11.19) and (11.20), for every $x \in \mathbb{R}^{N}, R>0$ and $u \in C^{2}\left(\overline{\Omega_{R}(x)}, \mathbb{R}\right)$ we have

$$
\lim _{r \rightarrow 0} \frac{m_{r}(u)(x)-u(x)}{q_{r}(x)}=\mathcal{L} u(x)=\lim _{r \rightarrow 0} \frac{M_{r}(u)(x)-u(x)}{Q_{r}(x)} .
$$

Indeed, for any fixed $u \in C^{2}\left(\overline{\Omega_{R}(x)}, \mathbb{R}\right)$ as above, let us prove that

$$
m_{r}(u)(x)-u(x)-\mathcal{L} u(x) q_{r}(x)=o\left(q_{r}(x)\right) \quad \text { as } r \rightarrow 0 .
$$

To this end, given $\varepsilon>0$, the continuity of $\mathcal{L} u$ and (3.2) ensure the existence of $\rho>0$ so small that $\rho<R$ and $\sup _{\Omega_{\rho}(x)}|\mathcal{L} u(y)-\mathcal{L} u(x)|<\varepsilon$. Hence, by (11.11), we have, for $r \in(0, \rho)$,

$$
\begin{array}{r}
\left|m_{r}(u)(x)-u(x)-\mathcal{L} u(x) q_{r}(x)\right|=\left|\int_{\Omega_{r}(x)}\left(\Gamma(x, y)-\frac{1}{r}\right)(\mathcal{L} u(y)-\mathcal{L} u(x)) \mathrm{d} y\right| \\
\leq \int_{\Omega_{r}(x)}\left(\Gamma(x, y)-\frac{1}{r}\right) \sup _{y \in \Omega_{\rho}(x)}|\mathcal{L} u(y)-\mathcal{L} u(x)| \mathrm{d} y \leq \varepsilon q_{r}(x) .
\end{array}
$$


Analogously, thanks to (11.18), one can show that

$$
M_{r}(u)(x)-u(x)-\mathcal{L} u(x) Q_{r}(x)=o\left(Q_{r}(x)\right) \quad \text { as } r \rightarrow 0,
$$

and (11.22) is completely proved.

We now exhibit alternative formulas for $q_{r}(x)$ and $Q_{r}(x)$, of independent interest. For every $x \in \mathbb{R}^{N}$, every $r>0$ (and every $\alpha>0$ ), we have

$$
\begin{aligned}
q_{r}(x) & =\int_{0}^{r} \frac{\left|\Omega_{s}(x)\right|}{s^{2}} \mathrm{~d} s, \\
Q_{r}(x) & =\int_{0}^{r} \frac{\left|\Omega_{s}(x)\right|}{s^{2}}\left(1-\left(\frac{s}{r}\right)^{\alpha+1}\right) \mathrm{d} s .
\end{aligned}
$$

Remark 11.2. These formulas obviously imply that, for every $x \in \mathbb{R}^{N}$, the functions $(0, \infty) \ni r \mapsto q_{r}(x), Q_{r}(x)$ are increasing.

To prove (11.23) and (11.24), we notice that the very definition (11.19) of $q_{r}(x)$ implies

$$
\begin{aligned}
q_{r}(x) & =\int_{\Omega_{r}(x)}\left(\Gamma(x, y)-\frac{1}{r}\right) \mathrm{d} y=\int_{\Gamma(x, y)>1 / r}\left(\int_{1 / r}^{\Gamma(x, y)} \mathrm{d} s\right) \mathrm{d} y \\
& =\int_{1 / r}^{\infty}\left(\int_{\Gamma(x, y)>s} \mathrm{~d} y\right) \mathrm{d} s=\int_{1 / r}^{\infty}\left|\Omega_{1 / s}(x)\right| \mathrm{d} s=\int_{0}^{r} \frac{\left|\Omega_{t}(x)\right|}{t^{2}} \mathrm{~d} t .
\end{aligned}
$$

Moreover, from (11.23) and (11.21) we get

$$
\begin{aligned}
Q_{r}(x) & =\frac{\alpha+1}{r^{\alpha+1}} \int_{0}^{r} \rho^{\alpha} q_{\rho}(x) \mathrm{d} \rho=\frac{\alpha+1}{r^{\alpha+1}} \int_{0}^{r} \rho^{\alpha}\left(\int_{0}^{\rho} \frac{\left|\Omega_{s}(x)\right|}{s^{2}} \mathrm{~d} s\right) \mathrm{d} \rho \\
& =\frac{\alpha+1}{r^{\alpha+1}} \int_{0}^{r}\left(\int_{s}^{r} \rho^{\alpha} \mathrm{d} \rho\right) \frac{\left|\Omega_{s}(x)\right|}{s^{2}} \mathrm{~d} s=\int_{0}^{r} \frac{\left|\Omega_{s}(x)\right|}{s^{2}}\left(1-\left(\frac{s}{r}\right)^{\alpha+1}\right) \mathrm{d} s .
\end{aligned}
$$

Note also that

$$
\lim _{r \rightarrow 0} q_{r}(x)=\lim _{r \rightarrow 0} Q_{r}(x)=0 .
$$

Indeed, $0 \leq q_{r}(x) \leq \int_{\Omega_{r}(x)} \Gamma(x, y) \mathrm{d} y \stackrel{r \rightarrow 0}{\longrightarrow} 0$, since $\Gamma(x, \cdot)$ is in $L_{\text {loc }}^{1}$ and $\Omega_{r}(x) \downarrow\{x\}$ as $r \rightarrow 0$. Finally, $\lim _{r \rightarrow 0} Q_{r}(x)=0$ follows from $\lim _{r \rightarrow 0} q_{r}(x)=0$ jointly with (11.21).

Next, we prove Theorem 3.4, stating that, for every $x \in \mathbb{R}^{N}, r, \alpha>0$ and for any $u \in C^{2}\left(\overline{\Omega_{r}(x)}, \mathbb{R}\right)$ one has

$$
m_{r}(u)(x)-M_{r}^{(\alpha)}(u)(x)=\frac{1}{\alpha r^{\alpha+1}} \int_{\Omega_{r}(x)}\left(r^{\alpha}-\Gamma^{-\alpha}(x, y)\right) \mathcal{L} u(y) \mathrm{d} y .
$$


Proof of Theorem 3.4. Let $x, r, \alpha$ and $u$ be as in the statement of Theorem 3.4. To prove (11.26), we need to go back to the Green formula (11.1). We choose $d$ as follows:

$$
d(y):=\Gamma^{-\alpha}(x, y), \quad y \in \mathbb{R}^{N} \backslash\{x\} .
$$

We also let $\Gamma_{x}(y):=\Gamma(x, y)$. For every $y \in \mathbb{R}^{N} \backslash\{x\}$ we have

$$
\begin{aligned}
\nabla d & =-\alpha \Gamma_{x}^{-\alpha-1} \nabla \Gamma_{x}, \\
\mathcal{L} d & =\operatorname{div}(A \nabla d)=-\alpha \operatorname{div}\left(\Gamma_{x}^{-\alpha-1} A \nabla \Gamma_{x}\right) \\
& =-\alpha \Gamma_{x}^{-\alpha-1} \operatorname{div}\left(A \nabla \Gamma_{x}\right)+\alpha(\alpha+1) \Gamma_{x}^{-\alpha-2}\left\langle A \nabla \Gamma_{x}, \nabla \Gamma_{x}\right\rangle
\end{aligned}
$$

(notice that $\operatorname{div}\left(A \nabla \Gamma_{x}\right)=\mathcal{L} \Gamma_{x}=0$ and recall the notation in (11.14))

$$
=\alpha(\alpha+1) \frac{\left\langle A \nabla \Gamma_{x}, \nabla \Gamma_{x}\right\rangle}{\Gamma_{x}^{\alpha+2}}=\alpha(\alpha+1) K(x, y) .
$$

Let $\alpha, r>0$ be fixed and suppose that $u$ is $C^{2}$ on an open neighborhood of $\overline{\Omega_{r}(x)}$. We set $R^{1 / \alpha}=r$ and we take any $\varepsilon \in(0, R)$. We next consider, in formula (11.1), the open set $\Omega=\{y: \varepsilon<d(y)<R\}$, where $\varepsilon \in(0, R)$ is arbitrary. This gives

$$
\begin{aligned}
\int_{\varepsilon<d<R}(u \mathcal{L} d-d \mathcal{L} u)= & \int_{d=R}(u\langle A \nabla d, \nu\rangle-d\langle A \nabla u, \nu\rangle) \mathrm{d} \sigma \\
& -\int_{d=\varepsilon}(u\langle A \nabla d, \nu\rangle-d\langle A \nabla u, v\rangle) \mathrm{d} \sigma \\
\stackrel{(11.2)}{=} & \int_{d=R} u\langle A \nabla d, \nu\rangle \mathrm{d} \sigma-\int_{d=\varepsilon} u\langle A \nabla d, \nu\rangle \mathrm{d} \sigma \\
& -R \int_{d<R} \mathcal{L} u+\varepsilon \int_{d<\varepsilon} \mathcal{L} u .
\end{aligned}
$$

On the other hand

$$
\int_{\varepsilon<d<R} d \mathcal{L} u-R \int_{d<R} \mathcal{L} u+\varepsilon \int_{d<\varepsilon} \mathcal{L} u=\int_{\varepsilon<d<R}(d-R) \mathcal{L} u-\int_{d<\varepsilon}(R-\varepsilon) \mathcal{L} u .
$$

Therefore we obtain

$$
\begin{aligned}
\int_{\varepsilon<d<R} u \mathcal{L} d= & \int_{d=R} u\langle A \nabla d, v\rangle \mathrm{d} \sigma-\int_{d=\varepsilon} u\langle A \nabla d, v\rangle \mathrm{d} \sigma \\
& +\int_{\varepsilon<d<R}(d-R) \mathcal{L} u-\int_{d<\varepsilon}(R-\varepsilon) \mathcal{L} u .
\end{aligned}
$$

From this equality, observing that $v=\nabla d /|\nabla d|=-\nabla \Gamma_{x} /\left|\nabla \Gamma_{x}\right|$ and recalling the expressions of $\nabla d$ and $\mathcal{L} d$ found a few lines above, we obtain

$$
\begin{aligned}
& \alpha(\alpha+1) \int_{\varepsilon<d<R} u(y) K(x, y) \mathrm{d} y=\int_{\varepsilon<d<R}(d-R) \mathcal{L} u-\int_{d<\varepsilon}(R-\varepsilon) \mathcal{L} u \\
& +\alpha \int_{d=R} u \Gamma_{x}^{-\alpha-1} \frac{\left\langle A \nabla \Gamma_{x}, \nabla \Gamma_{x}\right\rangle}{\left|\nabla \Gamma_{x}\right|} \mathrm{d} \sigma-\alpha \int_{d=\varepsilon} u \Gamma_{x}^{-\alpha-1} \frac{\left\langle A \nabla \Gamma_{x}, \nabla \Gamma_{x}\right\rangle}{\left|\nabla \Gamma_{x}\right|} \mathrm{d} \sigma
\end{aligned}
$$


(recall (11.6) and the fact that the set $\{d=R\}$ is equal to $\left\{\Gamma_{x}=R^{-1 / \alpha}\right\}$ )

$$
\begin{aligned}
= & \int_{\varepsilon<d<R}(d-R) \mathcal{L} u-\int_{d<\varepsilon}(R-\varepsilon) \mathcal{L} u \\
& +\alpha R^{(\alpha+1) / \alpha} \int_{d=R} u(y) \mathcal{K}(x, y) \mathrm{d} \sigma(y)-\alpha \varepsilon^{(\alpha+1) / \alpha} \int_{d=\varepsilon} u(y) \mathcal{K}(x, y) \mathrm{d} \sigma(y)
\end{aligned}
$$

(recall (11.5) and notice that $\{d=R\}$ is also the same as $\partial \Omega_{R^{1 / \alpha}}(x)$ )

$$
\begin{aligned}
= & \int_{\varepsilon<d<R}\left(\Gamma_{x}^{-\alpha}-R\right) \mathcal{L} u-\int_{d<\varepsilon}(R-\varepsilon) \mathcal{L} u \\
& +\alpha R^{(\alpha+1) / \alpha} m_{R^{1 / \alpha}}(u)(x)-\alpha \varepsilon^{(\alpha+1) / \alpha} m_{\varepsilon^{1 / \alpha}}(u)(x) .
\end{aligned}
$$

Thus,

$$
\begin{array}{r}
\alpha(\alpha+1) \int_{\varepsilon<d<R} u(y) K(x, y) \mathrm{d} y=\int_{\varepsilon<d<R}\left(\Gamma_{x}^{-\alpha}-R\right) \mathcal{L} u-\int_{d<\varepsilon}(R-\varepsilon) \mathcal{L} u \\
+\alpha R^{(\alpha+1) / \alpha} m_{R^{1 / \alpha}}(u)(x)-\alpha \varepsilon^{(\alpha+1) / \alpha} m_{\varepsilon^{1 / \alpha}}(u)(x) .
\end{array}
$$

We now let $\varepsilon \rightarrow 0$. Clearly, $\varepsilon^{(\alpha+1) / \alpha} m_{\varepsilon^{1 / \alpha}}(u)(x)$ vanishes, as $\varepsilon \rightarrow 0$, thanks to (11.10). The same is true of the integral of $(R-\varepsilon) \mathcal{L} u$ (a bounded function) over $\{d<\varepsilon\}=$ $\Omega_{\varepsilon^{1 / \alpha}}(x)$ (which shrinks to $\{x\}$ as $\varepsilon \rightarrow 0$ ). Finally, noticing that

$$
\{y: \varepsilon<d(y)<R\}=\Omega_{R^{1 / \alpha}}(x) \backslash \overline{\Omega_{\varepsilon^{1 / \alpha}}},
$$

we infer that (since $\Gamma_{x}^{-\alpha}$ is bounded by $R$ on $\Omega_{R^{1 / \alpha}}(x)$ )

$$
\int_{\varepsilon<d<R}\left(\Gamma_{x}^{-\alpha}-R\right) \mathcal{L} u \stackrel{\varepsilon \rightarrow 0}{\longrightarrow} \int_{\Omega_{R^{1 / \alpha}}(x)}\left(\Gamma_{x}^{-\alpha}-R\right) \mathcal{L} u .
$$

Furthermore, thanks to (11.15), for the left-hand side in (11.27) we have

$$
\begin{aligned}
(\alpha+1) \int_{\varepsilon<d<R} u(y) K(x, y) \mathrm{d} y & =R^{(\alpha+1) / \alpha} M_{R^{1 / \alpha}}(u)(x)-\varepsilon^{(\alpha+1) / \alpha} M_{\varepsilon^{1 / \alpha}}(u)(x) \\
& \stackrel{\varepsilon \rightarrow 0}{\longrightarrow} R^{(\alpha+1) / \alpha} M_{R^{1 / \alpha}}(u)(x), \quad \text { by (11.17). }
\end{aligned}
$$

Gathering together all the previous facts, and moving terms around in (11.27), we have finally proved the equality

$$
\alpha R^{(\alpha+1) / \alpha}\left(m_{R^{1 / \alpha}}(u)(x)-M_{R^{1 / \alpha}}(u)(x)\right)=\int_{\Omega_{R^{1 / \alpha}}(x)}\left(R-\Gamma_{x}^{-\alpha}\right) \mathcal{L} u .
$$

Replacing $R^{1 / \alpha}$ by $r$ and dividing by $\alpha r^{\alpha+1}$, we infer

$$
m_{r}(u)(x)-M_{r}(u)(x)=\frac{1}{\alpha r^{\alpha+1}} \int_{\Omega_{r}(x)}\left(r^{\alpha}-\Gamma_{x}^{-\alpha}\right) \mathcal{L} u,
$$

which is precisely (3.10). This ends the proof. 
With reference to the right-hand member in (11.26), we set

$$
\omega_{r}(x):=\frac{1}{\alpha r^{\alpha+1}} \int_{\Omega_{r}(x)}\left(r^{\alpha}-\Gamma^{-\alpha}(x, y)\right) \mathrm{d} y .
$$

Since $\Omega_{r}(x)$ equals $\left\{y: r^{\alpha}-\Gamma^{-\alpha}(x, y)>0\right\}$ (for any the positive $\alpha$ ), we have $\omega_{r}(x)>0$. Also, $\omega_{r}(x)$ is finite since $\Gamma^{-\alpha}$ is bounded by $r^{\alpha}$ on $\Omega_{r}(x)$.

Remark 11.3. In the notation of (11.28), for every $x \in \mathbb{R}^{N}, R, \alpha>0$, and $u \in$ $C^{2}\left(\overline{\Omega_{R}(x)}, \mathbb{R}\right)$ we have

$$
\lim _{r \rightarrow 0} \frac{m_{r}(u)(x)-M_{r}(u)(x)}{\omega_{r}(x)}=\mathcal{L} u(x) .
$$

This comes from representation formula (11.26) and the same arguments as in Remark 11.1 .

The following alternative representation of $\omega_{r}(x)$ holds:

$$
\omega_{r}(x)=\frac{1}{r^{\alpha+1}} \int_{0}^{r} s^{\alpha-1}\left|\Omega_{s}(x)\right| \mathrm{d} s .
$$

Indeed, starting from the definition of $\omega_{r}(x)$ in (11.28), we have

$$
\begin{aligned}
\omega_{r}(x) & =\frac{1}{\alpha r^{\alpha+1}} \int_{\Gamma_{x}>1 / r}\left(r^{\alpha}-\Gamma_{x}^{-\alpha}\right)=\frac{1}{r^{\alpha+1}} \int_{1 / \Gamma_{x}<r}\left(\int_{1 / \Gamma_{x}}^{r} s^{\alpha-1} \mathrm{~d} s\right) \mathrm{d} y \\
& =\frac{1}{r^{\alpha+1}} \int_{0}^{r} s^{\alpha-1}\left(\int_{1 / \Gamma_{x}<s} \mathrm{~d} y\right) \mathrm{d} s=\frac{1}{r^{\alpha+1}} \int_{0}^{r} s^{\alpha-1}\left|\Omega_{s}(x)\right| \mathrm{d} s .
\end{aligned}
$$

As a consequence of (3.3) and (11.30) we infer that, given any $x \in \mathbb{R}^{N}$,

$$
\lim _{r \rightarrow 0} \omega_{r}(x)=0 \text {. }
$$

Indeed, given $\varepsilon>0$, by (3.3) there exists $\rho>0$ such that $\left|\Omega_{r}(x)\right| / r<\varepsilon$ for every $r \in(0, \rho)$. As a consequence, for every $0<r<\rho$ we get (see (11.30))

$$
0 \leq \omega_{r}(x)=\frac{1}{r^{\alpha+1}} \int_{0}^{r} s^{\alpha} \frac{\left|\Omega_{s}(x)\right|}{s} \mathrm{~d} s \leq \frac{1}{r^{\alpha+1}} \int_{0}^{r} s^{\alpha} \varepsilon \mathrm{d} s=\frac{\varepsilon}{\alpha+1},
$$

thus proving (11.31).

Remark 11.4. For every $x \in \mathbb{R}^{N}$, the function $(0, \infty) \ni r \mapsto \omega_{r}(x)$ is continuous. This follows immediately from (11.30), taking into account that $s \mapsto s^{\alpha-1}\left|\Omega_{s}(x)\right|$ is locally summable on $[0, \infty)$, for $\left|\Omega_{s}(x)\right|$ is locally bounded and $s^{\alpha-1}$ is in $L_{\text {loc }}^{1}($ since $\alpha>0)$.

Remark 11.5. If $f(s):=\left|\Omega_{s}(x)\right| / s$ is an increasing function of $s>0$, then $r \mapsto \omega_{r}(x)$ is increasing too. This follows from the representation formula (11.30), by arguing as in Remark 5.6. The monotonicity of $\left|\Omega_{s}(x)\right| / s$ holds true in many interesting cases, such as in the Euclidean case of the Laplace operator in $\mathbb{R}^{N}, N \geq 3$, where $f(s)$ is a constant multiple of $s^{2 /(N-2)}$, and, more generally, in the case of the sub-Laplacians on Carnot groups (see (9.20), showing that $f(s)$ is a constant multiple of $\left.s^{2 /(Q-2)}\right)$. 
Acknowledgments. We would like to thank the anonymous referee of this paper whose valuable comments have allowed us to improve a former version of the manuscript.

\section{References}

[1] Armitage, D. H., Gardiner, S. J.: Classical Potential Theory. Springer Monogr. Math., Springer, London (2001) Zbl 0972.31001 MR 1801253

[2] Beckenbach, E. F., Radó, T.: Subharmonic functions and minimal surfaces. Trans. Amer. Math. Soc. 35, 648-661 (1933) Zbl 0007.12906 MR 1501707

[3] Blaschke, W.: Ein Mittelwertsatz und eine kennzeichnende Eigenschaft des logarithmischen Potentials. Leipz. Ber. 68, 3-7 (1916) JFM 46.0742.01

[4] Bonfiglioli, A., Cinti, C.: The theory of energy for sub-Laplacians with an application to quasi-continuity. Manuscripta Math. 118, 283-309 (2005) Zbl 1131.35006 MR 2183041

[5] Bonfiglioli, A., Lanconelli, E.: Liouville-type theorems for real sub-Laplacians. Manuscripta Math. 105, 111-124 (2001) Zbl 1016.35014 MR 1885817

[6] Bonfiglioli, A., Lanconelli, E.: Subharmonic functions on Carnot groups. Math. Ann. 325, 97-122 (2003) Zbl 1017.31003 MR 1957266

[7] Bonfiglioli, A., Lanconelli, E.: Gauge functions, eikonal equations and Bôcher's theorem on stratified Lie groups. Calc. Var. Partial Differential Equations 30, 277-291 (2007) Zbl 1137.31003 MR 2332415

[8] Bonfiglioli, A., Lanconelli, E.: A new characterization of convexity in free Carnot groups. Proc. Amer. Math. Soc. 140, 3263-3273 (2012) MR 2917098

[9] Bonfiglioli, A., Lanconelli, E., Uguzzoni, F.: Stratified Lie Groups and Potential Theory for their Sub-Laplacians. Springer Monogr. Math., Springer, New York (2007) Zbl 1128.43001 MR 2363343

[10] Bony, J.-M.: Principe du maximum, inégalité de Harnack et unicité du problème de Cauchy pour les opérateurs elliptiques dégénérés. Ann. Inst. Fourier (Grenoble) 19, no. 1, 277-304 (1969) Zbl 0176.09703 MR 0262881

[11] Citti, G., Garofalo, N., Lanconelli, E.: Harnack's inequality for sum of squares of vector fields plus a potential. Amer. J. Math. 115, 699-734 (1993) Zbl 0795.35018 MR 1221840

[12] Folland, G. B.: Subelliptic estimates and function spaces on nilpotent Lie groups. Ark. Mat. 13, 161-207 (1975) Zbl 0312.35026 MR 0494315

[13] Folland, G. B., Stein, E. M.: Hardy Spaces on Homogeneous Groups. Math. Notes 28, Princeton Univ. Press (1982) Zbl 0508.42025 MR 0657581

[14] Freitas, P., Matos J. P.: On the characterization of harmonic and subharmonic functions via mean-value properties. Potential Anal. 32, 189-200 (2010) Zbl 1187.31003 MR 2584983

[15] Garofalo, N., Lanconelli, E.: Asymptotic behavior of fundamental solutions and potential theory of parabolic operators with variable coefficients. Math. Ann. 283, 211-239 (1989) Zbl 0638.35003 MR 0980595

[16] Gaveau, L.: Principe de moindre action, propagation de la chaleur et estimées sous elliptiques sur certains groupes nilpotents. Acta Math. 139, 95-153 (1977) Zbl 0366.22010 MR 0461589

[17] Heinonen, J.: Lectures on Analysis on Metric Spaces. Universitext, Springer, New York (2001) Zbl 0985.46008 MR 1800917

[18] Hoh, W., Jacob, N.: Remarks on mean value properties of solutions of second order differential operators. Expo. Math. 9, 367-377 (1991) Zbl 0755.35005 MR 1137815

[19] Hörmander, L.: Notions of Convexity. Progr. Math. 127, Birkhäuser, Boston (1994) Zbl 0835.32001 MR 1301332 
[20] Juutinen, P., Lu, G., Manfredi, J. J., Stroffolini, B.: Convex functions on Carnot groups. Rev. Mat. Iberoamer. 23, 191-200 (2007) Zbl 1124.49024 MR 2351130

[21] Kozakiewicz, W.: Un théorème sur les opérateurs et son application à la théorie des Laplaciens généralisés. C. R. Soc. Sci. Varsovie 26, 18-24 (1933) JFM 59.1092.01 MR 2102746

[22] Lanconelli, E.: Maximum principles and symmetry results in sub-Riemannian settings. In: Symmetry for Elliptic PDEs, Contemp. Math. 528, Amer. Math. Soc., 17-33 (2010) Zbl 1232.35026 MR 2759032

[23] Lanconelli, E., Gutiérrez, C. E.: Classical, viscosity and average solutions for PDE's with nonnegative characteristic form. Rend. Lincei Mat. Appl. 15, 17-28 (2004) Zbl 1098.35052 MR 2102746

[24] Lu, G., Manfredi, J. J., Stroffolini, B.: Convex functions on the Heisenberg group. Calc. Var. Partial Differential Equations 19, 1-22 (2004) Zbl 1072.49019 MR 2027845

[25] Manfredi, J. J., Parviainen, M., Rossi, J. D.: An asymptotic mean value characterization for $p$-harmonic functions. Proc. Amer. Math. Soc. 138, 881-889 (2010) Zbl 1187.35115 MR 2566554

[26] Negrini, P., Scornazzani, V.: Wiener criterion for a class of degenerate elliptic operators. J. Differential Equations 66, 151-164 (1987) Zbl 0633.35018 MR 0871992

[27] Netuka, I., Veselý, J.: Mean value property and harmonic functions. In: Classical and Modern Potential Theory and Applications. NATO ASI Ser. Ser. C Math. Phys. Sci. 430, Kluwer, Dordrecht, 359-398 (1994) Zbl 0863.31012 MR 1321628

[28] Privaloff, I.: Sur les fonctions harmoniques. Rec. Math. Moscou (Mat. Sbornik) 32, 464-471 (1925) JFM 51.0363.02

[29] Reade, M.: Some remarks on subharmonic functions. Duke Math. J. 10, 531-536 (1943) Zbl 0063.06444 MR 0008447

[30] Rudin, W.: Real and Complex Analysis. McGraw-Hill, New York, 1966 Zbl 0142.01701 MR 0210528

[31] Saks, S.: On the operators of Blaschke and Privaloff for subharmonic functions. Rec. Math. Moscou (Mat. Sbornik) (2) 9, 451-456 (1941) Zbl 0024.40401 MR 0004363

[32] Stein, E. M.: Harmonic Analysis: Real-Variable Methods, Orthogonality, and Oscillatory Integrals. Princeton Math. Ser. 43, Princeton Univ. Press, Princeton (1993) Zbl 0821.42001 MR 1232192

[33] Trèves, F.: Topological Vector Spaces, Distributions and Kernels. Academic Press, New York (1967) Zbl 0171.10402 MR 0225131 